\title{
Anisotropic colloidal particles in critical fluids
}

\author{
E. Eisenriegler \\ Institut für Festkörperforschung, Forschungszentrum Jülich, D-52425 Jülich, Germany
}

(Received 17 March 2004; accepted 12 May 2004)

\begin{abstract}
We consider anisotropic colloidal particles with dumbbell or lens shapes that are immersed in a critical binary fluid mixture. The orientation-dependent long-ranged universal interactions mediated by the critical solvent between a particle and a wall or between two particles are investigated for mesoscopic particle sizes small compared to the correlation length and interparticle distances. Exact results are obtained using a "small particle operator expansion." The amplitudes of the isotropic and anisotropic operators in the expansion depend on the size and aspect ratio of the dumbbell or lens and are determined by density profiles in the Ising model at the critical point in a wedge geometry with symmetry-breaking fixed-spin boundary conditions. Dumbbells and ellipsoids with a symmetry preserving surface are also considered. (C) 2004 American Institute of Physics.
\end{abstract}

[DOI: $10.1063 / 1.1768514]$

\section{INTRODUCTION}

There are long-range solvation forces between colloidal particles dissolved in a complex fluid with a large correlation length, ${ }^{1,2}$ such as solutions of long flexible polymer chains, fluid mixtures near their critical demixing point, or ${ }^{4} \mathrm{He}$ near the superfluid transition. The particles locally disturb the critical complex fluid over a distance of the order of the correlation length, leading to a long-range interaction between the particles.

As in critical phenomena in the bulk ${ }^{3}$ or near surfaces, ${ }^{4,5}$ the solvation forces display a high degree of universality. In the asymptotic limit with particle size and correlation length $\xi$ much larger than microscopic lengths, apart from a few nonuniversal scale factors they are independent of microscopic details and only depend on a few gross properties of the fluid and the particles. ${ }^{6-8}$ For example, for particles in a one-component fluid near its liquid-vapor critical point or a binary mixture near its critical demixing point, the universal free energy of interaction between mesoscopic particles is the same as between corresponding mesoscopic holes cut into an Ising spin model with a "magnetic" field acting onto the surface spins of the holes. This field corresponds to the generic preference of a particle surface for one of the two phases (liquid or vapor, component 1 or component 2 rich) of the solvent. The field is absent in the exceptional case of a particle with no preference, in which case the surface locally weakens the tendency for demixing in the near-critical fluid. In the terminology of surface critical phenomena, these two types of surfaces are called "normal" and "ordinary" surfaces, ${ }^{5}$ respectively.

The second relevant property determining the universal critical interparticle interaction is the geometry (size and shape) of the mesoscopic particles. For spherical particles the fundamental quantity is the size ratio (particle radius) $/ \xi$. The size ratio dependence of the sphere-wall and sphere-sphere interaction in a critical fluid mixture has been analyzed in Refs. 9 and 10. The solvation forces of a near critical solvent are attractive for like particles and are a possible source of particle flocculation. However, to explain existing flocculation experiments in a near critical water-lutidine mixture, it seems necessary to take into account additional interactions, such as charge screening effects, as well. ${ }^{11}$

If the near critical fluid is a polymer solution, it induces a depletion interaction of long range. Polymers can also be discussed in terms of magnetic spin models, ${ }^{12}$ and in the above terminology the particle surface for nonadsorbing polymers corresponds to an ordinary surface. ${ }^{13}$

If the particles are not spheres but anisotropic, the interaction between them not only depends on the distance but also on the orientation. Both a force and a torque of long range are induced by the critical fluid. Anisotropic particles in solutions of nonadsorbing polymer chains are considered in Refs. 14-16. Here we consider anisotropic particles in a critical fluid mixture and study anisotropic effects both in the generic case of a normal particle surface (preference for one component) and the exceptional case of an ordinary surface (no preference). Not surprisingly, the results differ qualitatively in the two cases.

Particles with a large size ratio can be investigated by means of small curvature expansions of the Helfrich or Derjaguin type, see, e.g., Refs. 9, 10, and 17. Here we consider small anisotropic particles which are large on a microscopic scale ${ }^{18}$ but much smaller than interparticle distances and the correlation length $\xi$.

In the field theoretic description of the critical fluid in terms of the Ising model, the small particle is a perturbation which can be represented by a series of point operators. In addition to the series of isotropic operators used in Refs. $7-10$ to describe small spheres, we must now ${ }^{19}$ include anisotropic operators.

For simplicity we confine the discussion to particle shapes with a symmetry axis of revolution. This encompasses prolate or oblate ellipsoids and dumbbells composed of two touching spheres, shown in Fig. 1. Both types of particle shapes can be synthesized. ${ }^{20-22}$

We concentrate on the dumbbell shape, for which detailed predictions can be made. The reason is that a system at 


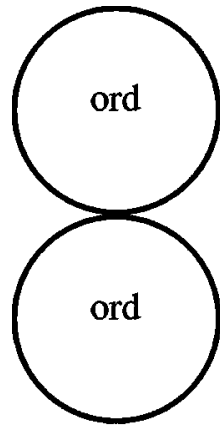

a)

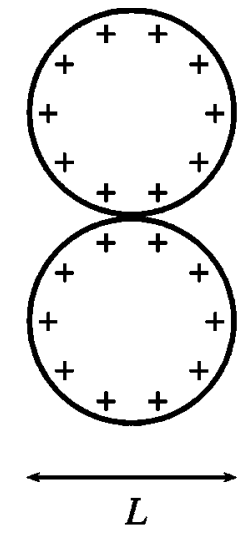

b)

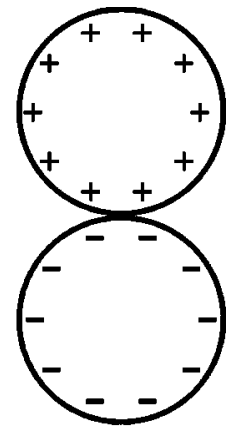

c)
FIG. 1. Dumbbell of tangentially touching spheres. (a) No preference for a particular component. (b) Both spheres prefer the same component. (c) The spheres prefer different components.

the critical point is not only scale invariant but, in general, also conformal invariant. ${ }^{23}$ The space outside the dumbbell in Fig. 1 can be obtained from the space inside two parallel plates by means of an inversion about a midpoint between the plates, which is a conformal, angle-preserving, transformation. Due to its high symmetry, much is known about a critical system between parallel plates ${ }^{24-26}$ and can be taken over to the dumbbell geometry.

In Sec. II the operator expansion for the dumbbells in Figs. 1(a)-1(c) is set up. We show that it describes both density profiles and multipoint functions in the presence of anisotropic particles. In Sec. III results from the Ising model in parallel plate geometry near $d=4$ and in $d=2$ spatial dimensions are used to check the expansion and to calculate the operator weights. We also compare with a particle of ellipsoidal shape. A connection between the small dumbbell and operator product expansions is established in Sec. II C 2 and checked in Secs. III A 2, III B, and III C 2. The particlewall and particle-particle interactions induced by the critical solvent are discussed in Sec. IV. In Sec. V we consider lenses and more general dumbbells composed of two overlapping spheres, which also have a symmetry axis of revolution. The results are summarized in Sec. VI. Some technical details are relegated to Appendixes A-D.

\section{OPERATOR EXPANSIONS FOR SMALL ANISOTROPIC PARTICLES, DUMBBELLS, AND CONFORMAL INVARIANCE}

\section{A. Small particle operator expansion}

Here we generalize earlier work describing the effect of small spherical particles in a critical fluid ${ }^{7,8}$ to the case of anisotropic particles. In the spirit of the operator-product expansion ${ }^{27}$ we represent a small mesoscopic perturbation of the critical system by a sum of point operators in the corresponding field theoretic Ising model.

We denote the Boltzmann factor of the particle by $e^{-\delta \mathcal{H}}$ and its bulk average at the critical point by $\left\langle e^{-\delta \mathcal{H}}\right\rangle_{\text {bulk }}$ $=e^{-\delta F}$, where $\delta F$ is the free energy per $k_{B} T$ for immersing the particle in the critical bulk system, right at the critical point. Then we expect

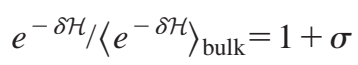

with

$$
\sigma=\sigma_{I}+\widetilde{\sigma}_{I}+\sigma_{A}+\widetilde{\sigma}_{A} .
$$

Here

$$
\sigma_{I}=a_{1} \epsilon+a_{2} \Delta \epsilon+a_{3} \lambda+\cdots
$$

and

$$
\widetilde{\sigma}_{I}=\widetilde{a}_{1} \varphi+\widetilde{a}_{2} \Delta \varphi+\cdots
$$

are linear combinations of isotropic (I) point operators which are even and odd under the Ising up-down symmetry, and we explicitly show the leading terms, with $\epsilon$ the energy density and $\varphi$ the order parameter. Note that only isotropic spatial derivatives such as the Laplacian $\Delta$ enter in Eqs. (2.3) and (2.4). In Eq. (2.3) we also show the isotropic even operator $\lambda$ of lowest scaling dimension other than the energy density $\epsilon$ or a derivative thereof. On approaching the upper critical dimension $d=4$, the scaling dimension $x_{\lambda}=d+\omega$ of $\lambda$, with $\omega$ the correction to scaling exponent, tends to 4 and becomes degenerate with the scaling dimension $x_{\epsilon}+2=d$ $-(1 / \nu)+2$ of $\Delta \epsilon$. The quantities $\sigma_{A}$ and $\widetilde{\sigma}_{A}$ in Eq. (2.2) are linear combinations of even and odd anisotropic operators, discussed below.

Since the left-hand side of Eq. (2.1) has vanishing scaling dimension, the amplitudes $a, \widetilde{a}$ (and the amplitudes $b, \widetilde{b}$ of anisotropic operators below) for particles large on the microscopic scale are proportional to the particle size raised to the scaling dimension $d_{\mathcal{O}}$ of the accompanying operator $\mathcal{O}$. The leading contributions for particles small compared to the other mesoscopic lengths come from the operators with the lowest scaling dimensions.

For particles with an up-down symmetry-preserving "ordinary" surface (the exceptional case for a particle in a binary fluid mixture), only even operators appear in Eq. (2.1), and the amplitudes $\widetilde{a}$ in Eq. (2.4) and corresponding amplitudes $\widetilde{b}$ for odd anisotropic operators vanish. For particles with a "normal" surface, which prefers one of the components and breaks the up-down symmetry (the generic case), both odd and even operators appear ${ }^{7-10}$ in Eq. (2.1). For a spherical particle only isotropic operators are included in Eq. (2.1) while for anisotropic particles there are both anisotropic and isotropic operators.

For spherical particles the amplitudes $a_{1}, \widetilde{a}_{1}$ are given in Refs. 7 and 8, and we calculate $a_{2}, a_{3}$, and $\widetilde{a}_{2}$ in Eqs. (2.20)-(2.24) below.

Our main concern is anisotropic particles with a symmetry axis of revolution. If, in addition, the particle is reflection symmetric about its center, for example, prolate or oblate ellipsoids of revolution or the dumbbells in Figs. 1(a) and 1(b), the leading even and odd anisotropic operators are

$$
\sigma_{A}=b_{1} \partial_{\|}^{2} \epsilon+b_{2} T_{\|,\|}+\cdots
$$

and

$$
\widetilde{\sigma}_{A}=\widetilde{b}_{1} \partial_{\|}^{2} \varphi+\cdots .
$$


Here $\partial_{\|}=\Sigma_{\mu} \varrho_{\mu} \partial_{\mu}$ is a derivative along the axis of revolution characterized by the unit vector $\vec{\varrho}$, and $T_{\|,\|}=\Sigma_{\mu, \nu} \varrho_{\mu} \varrho_{\nu} T_{\mu \nu}$ is the diagonal component of the stress tensor ${ }^{23,28}$ of the Ising field theory parallel to the axis. In the case of an ellipsoid the amplitudes $a, \widetilde{a}, b, \widetilde{b}$ depend on the particle size and the aspect ratio (the ratio between the lengths of the large and small axes of the ellipsoid), see Ref. 16 and Sec. III B below. In Sec. V we consider dumbbells of various aspect ratios.

We now give the explicit form of the operator expansions for the dumbbell-shaped particles of Fig. 1. The two dumbbells of Fig. 1(a) or 1(b) with symmetry preserving (ord, ord) or symmetry breaking $(+,+)$ boundary conditions are reflection symmetric about their center. Thus the two Ising-spin configurations $s(\mathbf{r})$ and $s(-\mathbf{r})$ have the same probability, and Eqs. (2.1)-(2.6) apply. For the (-, +) dumbbell of Fig. 1(c), the two configurations $s(\mathbf{r})$ and $-s$ $(-\mathbf{r})$ have the same probability. ${ }^{29}$ In the $(-,+)$ case there is no odd isotropic contribution, and the odd anisotropic operators must be accompanied by an odd number of spatial derivatives. Thus Eqs. (2.4) and (2.6) are replaced by

$$
\widetilde{\sigma}_{I}=0
$$

and

$$
\widetilde{\sigma}_{A}=\widetilde{c}_{1} \partial_{\|} \varphi+\cdots .
$$

The small particle operator expansion can be used at and near the critical point as long as the particle size is much smaller than the correlation length $\xi$. Away from $T_{c}$ there are additional operators in $\sigma$, such ${ }^{16}$ as $t \epsilon$ with $t \propto T-T_{c}$, but these are accompanied by higher powers of the particle size (power exponent $d$ in the above example). The leading isotropic and anisotropic small particle effects are determined, in general, by the operators and their amplitudes which appear in the expansion at the critical point.

\section{B. Results for dumbbells}

For the three dumbbells in Fig. 1 with $(i, j)$ $=($ ord, ord $),(+,+)$, and $(-,+)$, all the amplitudes $a^{(i, j)}, b^{(i, j)}, c^{(i, j)}$ defined in the preceding section can be expressed in terms of density profiles of the critical system at the bulk critical point in the parallel plate geometry.

The contributions in Eqs. (2.3) and (2.5) which are related to the energy density $\epsilon$ are given by

$$
\begin{aligned}
a_{1}^{(i, j)} \epsilon & +a_{2}^{(i, j)} \Delta \epsilon+b_{1}^{(i, j)} \partial_{\|}^{2} \epsilon \\
= & (L / 2)^{x_{\epsilon}}\left\{\mathcal{E}_{i, j}(0) \epsilon+L^{2} \mathcal{E}_{i, j}^{\prime \prime}(0) \frac{1}{8 x_{\epsilon}\left(x_{\epsilon}+1\right)}\right. \\
& \left.\times\left[\left(\frac{1}{2 x_{\epsilon}+2-d} \Delta \epsilon\right)+\partial_{\|}^{2} \epsilon\right]\right\} / B_{\epsilon}^{1 / 2}
\end{aligned}
$$

for the dumbbell. Here $x_{\epsilon}=d-(1 / \nu)$ is the scaling dimension of the energy density, $B_{\epsilon}$ is the nonuniversal amplitude of the critical bulk two-point function $\langle\epsilon(\mathbf{r}) \epsilon(0)\rangle_{\text {bulk }}$, and $\mathcal{E}_{i, j}$ is the universal scaling function of the energy density profile $\langle\epsilon(\hat{\mathbf{r}})\rangle_{\text {film }}^{(i, j)}$ in a film between parallel plates.

For the operator $\epsilon$ and the two other isotropic operators $\lambda$ and $\varphi$ considered below, we define the bulk amplitudes $B$ by

$$
\langle\mathcal{O}(\mathbf{r}) \mathcal{O}(0)\rangle_{\text {bulk }}=B_{\mathcal{O}} r^{-2 x_{\mathcal{O}}}, \quad \mathcal{O}=\epsilon, \lambda, \varphi,
$$

and the universal scaling functions $\mathcal{P}$ of the film profiles at the bulk critical point by

$$
\langle\mathcal{O}(\hat{\mathbf{r}})\rangle_{\text {film }}^{(i, j)}=B_{\mathcal{O}}^{1 / 2}(2 L)^{-x_{\mathcal{O}}} \mathcal{P}_{i, j}(\hat{z} / L)
$$

with

$$
\mathcal{P}=\mathcal{E}, \mathcal{L}, \mathcal{F} \quad \text { for } \quad \mathcal{O}=\epsilon, \lambda, \varphi .
$$

Here the film is confined between parallel plates at $(\hat{z}$ $=-L, \hat{z}=+L)$ with boundary conditions $(i, j)=$ (ord,ord $)$, $(+,+)$, or $(-,+)$ on the plates and $\hat{\mathbf{r}}=\left(\hat{\mathbf{r}}_{\|}, \hat{z}\right)$. For (ord,ord) and $(+,+)$ plates the two Ising spin configurations $s\left(\hat{\mathbf{r}}_{\|}, \hat{z}\right)$, $s\left(\hat{\mathbf{r}}_{\|},-\hat{z}\right)$ have the same probabilities, while for $(-,+)$ the configurations $s\left(\hat{\mathbf{r}}_{\|}, \hat{z}\right),-s\left(\hat{\mathbf{r}}_{\|},-\hat{z}\right)$. Thus in all three cases the scaling functions $\mathcal{E}_{i, j}$ and $\mathcal{L}_{i, j}$ of the even operators $\epsilon$ and $\lambda$ are even functions of the argument $\hat{z} / L$. For the odd operator $\varphi$ only the scaling function $\mathcal{F}_{++}$is even and nonvanishing, while $\mathcal{F}_{-+}$is odd, and $\mathcal{F}_{\text {ord,ord }}$ vanishes.

The small dumbbell amplitudes follow from the behavior of $\mathcal{P}(y)$ near the center of the film, where $|y| \ll 1$. For later use we also note the asymptotic form

$$
\mathcal{P}_{i, j}(y) \rightarrow 2^{x_{\mathcal{O}}}\left[\mathcal{A}_{\mathcal{O}}^{(i)}(1+y)^{-x_{\mathcal{O}}}, \mathcal{A}_{\mathcal{O}}^{(j)}(1-y)^{-x_{\mathcal{O}}}\right]
$$

near the planar boundaries, where $y \rightarrow[-1,1]$. Here the universal amplitude $\mathcal{A}_{\mathcal{O}}$ describes the dependence $\mathcal{A}_{\mathcal{O}} / z^{x_{\mathcal{O}}}$ on the distance $z$ from the planar boundary wall of the normalized profile $\langle\mathcal{O}\rangle_{\mathrm{hs}} / B_{\mathcal{O}}^{1 / 2}$ in the half space (hs).

Similar to Eq. (2.9) the amplitude $a_{3}$ of the isotropic and even operator $\lambda$ is given by

$$
a_{3}^{(i, j)} \lambda=(L / 2)^{x_{\lambda}} \mathcal{L}_{i, j}(0) \lambda / B_{\lambda}^{1 / 2}
$$

with the film profile scaling function $\mathcal{L}$ defined in Eqs. (2.11) and (2.12).

The contribution of the stress tensor is

$$
b_{2}^{(i, j)} T_{\|,\|}=(L / 2)^{d} \Delta_{i, j} \frac{d}{B_{T}} T_{\|,\|},
$$

where $\Delta_{i, j}$ is the universal amplitude in the singular contribution $(2 L)^{1-d} \Delta_{i, j}$ of the free energy per $k_{B} T$ per unit area of the film. This amplitude also appears in the average ${ }^{30}$

$$
\left\langle T_{\hat{z}, \hat{z}}(\hat{\mathbf{r}})\right\rangle_{\text {film }}^{(i, j)}=(2 L)^{-d}(d-1) \Delta_{i, j}
$$

of the stress tensor component $T_{\hat{z}, \hat{z}}$, where the $\hat{z}$ axis is perpendicular to the film boundaries. The quantity $B_{T}$ is the universal amplitude in the bulk two-point correlation ${ }^{24,31}$

$$
\begin{aligned}
\left\langle T_{k, l}(\mathbf{r}) T_{m, n}(0)\right\rangle_{\mathrm{bulk}}= & B_{T} r^{-2 d}\left\{(1 / 2)\left[I_{k, m} I_{l, n}+I_{k, n} I_{l, m}\right]\right. \\
& \left.-(1 / d) \delta_{k, l} \delta_{m, n}\right\}
\end{aligned}
$$

of the stress tensor at the critical point, with $I_{k, m}=\delta_{k, m}$ $-2 r_{k} r_{m} / r^{2}$.

We now turn to the contributions in the small dumbbell operator expansion which are odd in the Ising up-down symmetry and only appear for the symmetry breaking $(+,+)$ and $(-,+)$ dumbbells of Figs. 1(b) and 1(c). For the $(+,+)$ dumbbell these have the form (2.4) and (2.6), with 


$$
\begin{aligned}
\tilde{a}_{1} \varphi+ & \tilde{a}_{2} \Delta \varphi+\tilde{b}_{1} \partial_{\|}^{2} \varphi \\
= & (L / 2)^{x}{ }_{\varphi}\left\{\mathcal{F}_{++}(0) \varphi+L^{2} \mathcal{F}_{++}^{\prime \prime}(0) \frac{1}{8 x_{\varphi}\left(x_{\varphi}+1\right)}\right. \\
& \left.\times\left[\left(\frac{1}{2 x_{\varphi}+2-d} \Delta \varphi\right)+\partial_{\|}^{2} \varphi\right]\right\} / B_{\varphi}^{1 / 2} .
\end{aligned}
$$

Equation (2.18) has the same structure as Eq. (2.9) except that $\epsilon, x_{\epsilon}, B_{\epsilon}$, and $\mathcal{E}_{i, j}$ are replaced by $\varphi$, the order parameter exponent $x_{\varphi}=\beta / \nu=(d-2+\eta) / 2$, the nonuniversal bulk amplitude $B_{\varphi}$, and the universal scaling function $\mathcal{F}_{++}$of the order parameter profile in a film with $(+,+)$ boundary conditions, which are defined in Eqs. (2.10) and (2.11).

For the $(-,+)$ dumbbell the odd contributions are given by Eqs. (2.7) and (2.8), with

$$
\widetilde{c}_{1} \partial_{\|} \varphi=(L / 2)^{x_{\varphi}+1} \mathcal{F}_{-,+}^{\prime}(0) \frac{1}{x_{\varphi}} \partial_{\|} \varphi / B_{\varphi}^{1 / 2} .
$$

Note that the - and + spheres of the $(-,+)$ dumbbell are situated on the negative and positive side of the $\|$ axis.

The results (2.9) - (2.19) for dumbbells should be compared with those for spherical particles. In the latter case all the amplitudes $b_{1}, b_{2}, \tilde{b}_{1}$ of anisotropic operators vanish, the weights of even isotropic operators are given by

$$
\begin{aligned}
& a_{1}^{(i)} \epsilon+a_{2}^{(i)} \Delta \epsilon=S_{\epsilon}^{(i)}+S_{\Delta \epsilon}^{(i)}, \\
& a_{3}^{(i)} \lambda=S_{\lambda}^{(i)},
\end{aligned}
$$

for an ordinary $(i=$ ord) or symmetry breaking $(i=+)$ surface, and of odd operators by

$$
\widetilde{a}_{1}^{(+)} \varphi+\widetilde{a}_{2}^{(+)} \Delta \varphi=S_{\varphi}^{(+)}+S_{\Delta \varphi}^{(+)} .
$$

Here

$$
S_{\mathcal{O}}^{(i)}=\mathcal{A}_{\mathcal{O}}^{(i)} D^{x} \mathcal{O} \mathcal{O} / B_{\mathcal{O}}^{1 / 2}
$$

and

$$
S_{\Delta \mathcal{O}}^{(i)}=\mathcal{A}_{\mathcal{O}}^{(i)} D^{x_{\mathcal{O}}+2} \frac{1 / 8}{2 x_{\mathcal{O}}+2-d} \Delta \mathcal{O} / B_{\mathcal{O}}^{1 / 2}
$$

with $D$ the diameter of the sphere and $\mathcal{A}_{\mathcal{O}}^{(i)}$ the universal amplitudes of density profiles in the half space introduced below Eq. (2.13).

In order to compare $(i, i)$ dumbbells with single $(i)$ spheres, note that due to Eqs. (2.9), (2.14), (2.18), and $(2.20)-(2.23)$ the ratios $a_{1}^{(i, i)} / a_{1}^{(i)}, a_{3}^{(i, i)} / a_{3}^{(i)}$, and $\tilde{a}_{1}^{(+,+)} / \widetilde{a}_{1}^{(+)}$of weights of the isotropic operators $\mathcal{O}=\epsilon, \lambda$, and $\varphi$ have the form $(L / D)^{x} \mathcal{O}\left[\mathcal{P}_{i, i}(0) /\left(2^{x} \mathcal{O} \mathcal{A}_{\mathcal{O}}^{(i)}\right)\right]$. For single spheres with the size $D=L$ of one of the two spheres composing the dumbbells in Figs. 1(a) and 1(b) one expects weaker weights than for the dumbbell, i.e., the square bracket in the above form should be larger than 1. This is plausible, since the actual profiles $\mathcal{P}_{i, i}(0)$ in the midplane of an $(i, i)$ film should have a larger modulus than the extrapolation of the half space profiles in Eq. (2.13). Conversely for a single sphere with the size $D=2 L$, one expects a greater weight than for the dumbbell it geometrically circumscribes. Indeed, using the results for $d$ near 4 and $d=2$ given in Sec.
III, one may verify that the corresponding weight ratios $2^{-2 x} \epsilon \mathcal{E}_{\text {ord,ord }}(0) / \mathcal{A}_{\epsilon}^{(\text {ord })}$ and $2^{-2 x} \varphi \mathcal{F}_{++}(0) / \mathcal{A}_{\varphi}^{(+)}$are smaller than 1 .

\section{Derivation}

To derive the results (2.9)-(2.19), we use the inversion

$$
\hat{\mathbf{r}} \equiv\left(\hat{\mathbf{r}}_{\|}, \hat{z}\right)=\frac{\mathbf{r}}{r^{2}} L^{2}, \quad \mathbf{r} \equiv\left(\mathbf{r}_{\perp}, r_{\|}\right),
$$

which is a conformal, angle-preserving transformation, to map the dumbbell geometry of Fig. 1 in $\mathbf{r}$ space onto the film geometry in $\hat{\mathbf{r}}$ space. The surfaces of the two spheres of diameter $L$ with centers on the negative and positive $r_{\|}$axis are mapped onto the planar boundaries $\hat{z}=-L$ and $+L$ of the film. Here $\mathbf{r}_{\perp}$ and $r_{\|}$are the components of $\mathbf{r}$ perpendicular and parallel to the dumbbell axis of revolution, and $\hat{\mathbf{r}}_{\|}$and $\hat{z}$ are the components of $\hat{\mathbf{r}}$ parallel and perpendicular to the boundary planes. The conformal mapping is justified, ${ }^{18}$ provided the sphere diameter $L$ is much larger than the extrapolation length $\ell$ and provided one considers density profiles or correlation functions at points farther from the spherical surfaces than $\ell$.

\section{Density profiles}

First we consider density profiles $\langle\mathcal{O}(\mathbf{r})\rangle_{\mathrm{DB}}^{(i, j)}$ in the presence of a dumbbell (DB) with boundary conditions $(i, j)$ $=($ ord, ord $),(+,+)$, or $(-,+)$, as in Fig. 1. Due to conformal invariance at the critical point, ${ }^{23}$ density profiles of scalar operators $\mathcal{O}$ such as the energy density $\epsilon$ or the order parameter $\varphi$ follow from the corresponding profile (2.11) in the film via

$$
\langle\mathcal{O}(\mathbf{r})\rangle_{\mathrm{DB}}^{(i, j)}=b(\hat{\mathbf{r}})^{x_{\mathcal{O}}}\langle\mathcal{O}(\hat{\mathbf{r}})\rangle_{\text {film }}^{(i, j)},
$$

where $b$ is the local dilatation factor

$$
b(\hat{\mathbf{r}})=|\operatorname{det}(\partial \hat{\mathbf{r}} / \partial \mathbf{r})|^{1 / d}=L^{2} / r^{2}
$$

of the transformation (2.25). Substituting Eq. (2.11) into Eq. (2.26) leads to

$$
\langle\mathcal{O}(\mathbf{r})\rangle_{\mathrm{DB}}^{(i, j)} / B_{\mathcal{O}}^{1 / 2}=\left(\frac{L}{2 r^{2}}\right)^{x_{\mathcal{O}}} \mathcal{P}_{i, j}\left(r_{\|} L / r^{2}\right)
$$

with the expansion

$$
\begin{aligned}
\langle\mathcal{O}(\mathbf{r})\rangle_{\mathrm{DB}}^{(i, i)} / B_{\mathcal{O}}^{1 / 2}= & \left(\frac{L}{2 r^{2}}\right)^{x_{\mathcal{O}}}\left[\mathcal{P}_{i, i}(0)+\frac{1}{2}\left(\frac{r_{\|} L}{r^{2}}\right)^{2}\right. \\
& \left.\times \mathcal{P}_{i, i}^{\prime \prime}(0)+\cdots\right]
\end{aligned}
$$

for the symmetric case $i=j$ and a distance $r$ from the dumbbell center much larger than the size $L$ of the dumbbell. We now verify that this result is reproduced by the small dumbbell expansion (2.1), which predicts

$$
\langle\mathcal{O}(\mathbf{r})\rangle_{\mathrm{DB}}^{(i, j)}=\frac{\langle\mathcal{O}(\mathbf{r})(1+\sigma)\rangle_{\text {bulk }}}{\langle 1+\sigma\rangle_{\text {bulk }}} .
$$

Since at the critical point both bulk one-point averages such as $\langle\sigma\rangle_{\text {bulk }}$ and $\langle\mathcal{O}(\mathbf{r})\rangle_{\text {bulk }}$ and "nondiagonal" two-point functions such as $\langle\epsilon(\mathbf{r}) \varphi(0)\rangle_{\text {bulk }},\langle\epsilon(\mathbf{r}) \lambda(0)\rangle_{\text {bulk }}$, or $\left\langle\epsilon(\mathbf{r}) T_{\|,\|}(0)\right\rangle_{\text {bulk }}$ vanish, Eq. (2.30) leads to 


$$
\langle\epsilon(\mathbf{r})\rangle_{\mathrm{DB}}^{(i, j)}=\left\langle\epsilon(\mathbf{r})\left[a_{1} \epsilon+a_{2} \Delta \epsilon+b_{1} \partial_{\|}^{2} \epsilon\right]\right\rangle_{\text {bulk }} .
$$

Taking derivatives of the bulk two-point function $\langle\epsilon \epsilon\rangle_{\text {bulk }}$ in Eq. (2.10), one sees that the operator expansion Eq. (2.31) with $a_{1}, a_{2}, b_{1}$ defined in (2.9) does indeed reproduce the expansion (2.29) of the 'direct' result (2.28) for the energy density profile.

For the order parameter density profiles near $(+,+)$ and $(-,+)$ dumbbells, Eqs. (2.26) $-(2.30)$ with $\mathcal{O}=\varphi$ lead to

$$
\begin{aligned}
\langle\varphi(\mathbf{r})\rangle_{\mathrm{DB}}^{(+,+)} / B_{\varphi}^{1 / 2}= & \left(\frac{L}{2 r^{2}}\right)^{x_{\varphi}}\left[\mathcal{F}_{+,+}(0)\right. \\
& \left.+\frac{1}{2}\left(\frac{r_{\|} L}{r^{2}}\right)^{2} \mathcal{F}_{+,+}^{\prime \prime}(0)+\cdots\right]
\end{aligned}
$$

and

$$
\langle\varphi(\mathbf{r})\rangle_{\mathrm{DB}}^{(-,+)} / B_{\varphi}^{1 / 2}=\left(\frac{L}{2 r^{2}}\right)^{x_{\varphi}} \frac{r_{\|} L}{r^{2}} \mathcal{F}_{-,+}^{\prime}(0)+\cdots .
$$

Again the operator expansion reproduces these results. On replacing $\epsilon$ by $\varphi$ in Eq. (2.30), the expansion (2.2)-(2.8) predicts

$$
\langle\varphi(\mathbf{r})\rangle_{\mathrm{DB}}^{(+,+),(-,+)}=\left\langle\varphi(\mathbf{r})\left[\widetilde{a}_{1} \varphi+\widetilde{a}_{2} \Delta \varphi+\widetilde{b}_{1} \partial_{\|}^{2} \varphi, \widetilde{c}_{1} \partial_{\|} \varphi\right]\right\rangle_{\text {bulk }}
$$

for the $[(+,+),(-,+)]$ dumbbells, which on using Eq. (2.18) or Eq. (2.19) and the order parameter bulk two-point function (2.10) indeed agrees with Eq. (2.32) or Eq. (2.33).

Finally we consider the density profile of the stress tensor

$$
\begin{aligned}
\left\langle T_{k, l}(\mathbf{r})\right\rangle_{\mathrm{DB}}^{(i, j)}= & \left(\frac{L}{2 r^{2}}\right)^{d} \Delta_{i, j}\left[-\delta_{k, l}+d\left(\delta_{k, \|}-2 \frac{r_{k} r_{\|}}{r^{2}}\right)\right. \\
& \left.\times\left(\delta_{l, \|}-2 \frac{r_{l} r_{\|}}{r^{2}}\right)\right]
\end{aligned}
$$

in the presence of a dumbbell with $(i, j)=($ ord, ord $),(+,+)$, or $(-,+)$ boundary conditions, as in Fig. 1. This follows from the average (2.16) of $T_{\hat{z}, \hat{z}}$ in the parallel plate geometry, the corresponding values $(2 L)^{-d}(-1) \Delta_{i, j}$ if both tensor components are parallel to the plates (so that $T$ is traceless), and 0 otherwise. Here one uses the inversion transformation

$$
\left\langle T_{k, l}(\mathbf{r})\right\rangle_{\mathrm{DB}}^{(i, j)}=b(\hat{\mathbf{r}})^{d} \sum_{\alpha, \beta} \mathcal{R}_{k, \alpha} \mathcal{R}_{l, \beta}\left\langle T_{\alpha, \beta}(\hat{\mathbf{r}})\right\rangle_{\text {film }}^{(i, j)} .
$$

Due to the tensor character of $T$, Eq. (2.36) is slightly more complicated than the transformation (2.26) of scalar operators, since it involves both the dilatation factor $b$ in Eq. (2.27) and the local rotation ${ }^{24}$

$$
\mathcal{R}_{k, \alpha}(\hat{\mathbf{r}})=\delta_{k, \alpha}-2 \frac{r_{k} r_{\alpha}}{r^{2}},
$$

which is contained in the inversion (2.25). The result (2.35) agrees with the prediction

$$
\left\langle T_{k, l}(\mathbf{r})\right\rangle_{\mathrm{DB}}^{(i, j)}=\left\langle T_{k, l}(\mathbf{r}) b_{2} T_{\|,\|}\right\rangle_{\text {bulk }}
$$

of the operator expansion with $b_{2}$ defined in Eq. (2.15). This follows from the form (2.17) of the bulk correlation of the stress tensor. While the stress tensor density profile outside an anisotropic particle is generally nonvanishing, see the expressions (3.40) and (3.43) for an ellipsoid in Sec. III B, it vanishes for an isotropic spherical particle.

\section{Two-point functions}

A small particle operator expansion describes the changes of arbitrary multipoint functions of the near critical system on immersing the particle. Besides the one-point functions or density profiles discussed above, we now check whether Eqs. (2.9), (2.15), (2.18), and (2.19) describe the changes of the two point functions $\left\langle\varphi\left(\mathbf{r}_{1}\right) \varphi\left(\mathbf{r}_{2}\right)\right\rangle_{\mathrm{DB}}$, $\left\langle\epsilon\left(\mathbf{r}_{1}\right) \epsilon\left(\mathbf{r}_{2}\right)\right\rangle_{\mathrm{DB}}$, and the mixed function $\left\langle\varphi\left(\mathbf{r}_{1}\right) \epsilon\left(\mathbf{r}_{2}\right)\right\rangle_{\mathrm{DB}}$. Again the checks are simplest right at the critical point, ${ }^{32}$ since conformal transformations such as (2.25) can be used. Like the order parameter profile $\langle\varphi(\mathbf{r})\rangle_{\mathrm{DB}}$, the mixed function at the critical point only exists for $(+,+)$ and $(-,+)$ dumbbells in Figs. 1(b) and 1(c) and vanishes due to the Ising up-down symmetry for the (ord, ord) dumbbell of Fig. 1(a). The validity of the expansion in the presence of distant perturbations such as a planar boundary wall will be tested in Sec. IV.

Two points $\mathbf{r}_{1}, \mathbf{r}_{2}$ which are much farther from the dumbbell than its size $L$ are mapped by the inversion (2.25) onto points $\hat{\mathbf{r}}_{1}, \hat{\mathbf{r}}_{2}$ with separation much smaller than their distance from the two planar walls in the film geometry. This is a situation in which the well-known operator product expansion of Wilson, Polyakov, and Kadanoff applies. At the critical point the expansion for the product $\varphi \varphi$ of two order parameters or the product $\epsilon \epsilon$ of two energy densities is given by $^{24,31,33}$

$$
\begin{aligned}
\mathcal{O}\left(\hat{\mathbf{r}}_{1}\right) \mathcal{O}\left(\hat{\mathbf{r}}_{2}\right)-\frac{B_{\mathcal{O}}}{s^{2 x_{\mathcal{O}}}}= & \frac{C_{\mathcal{O O} \epsilon} / B_{\epsilon}}{s^{2 x_{\mathcal{O}^{-x}}}} \mathcal{D}_{0, x}\left(\mathbf{s}, \partial_{\hat{\mathbf{r}}}\right) \epsilon(\hat{\mathbf{r}}) \\
& +\frac{C_{\mathcal{O O} T} / B_{T}}{s^{2 x_{\mathcal{O}}-d+2}} \sum_{\mu, \nu} s_{\mu} s_{\nu} T_{\mu \nu}(\hat{\mathbf{r}}) \\
& +\frac{C_{\mathcal{O O} \lambda} / B_{\lambda}}{s^{2 x_{\mathcal{O}}-x_{\lambda}}} \lambda(\hat{\mathbf{r}})+\cdots,
\end{aligned}
$$

in the long distance limit, with $\mathcal{O}$ denoting either $\varphi$ or $\epsilon$, the short distance vector

$$
\mathbf{s}=\hat{\mathbf{r}}_{1}-\hat{\mathbf{r}}_{2} \equiv \hat{\mathbf{r}}_{12},
$$

$\mathcal{D}$ is a differential operator defined in Eq. (A1), and the point

$$
\hat{\mathbf{r}}=\left(\hat{\mathbf{r}}_{1}+\hat{\mathbf{r}}_{2}\right) / 2
$$

is chosen so that no first derivative appears in $\mathcal{D}_{0, x_{\epsilon}}$. The amplitudes $C_{\mathcal{O O} \epsilon}, C_{\mathcal{O O} \lambda}$, and $C_{\mathcal{O O T}}$ characterize bulk threepoint functions of $\mathcal{O}, \mathcal{O}, \epsilon$, of $\mathcal{O}, \mathcal{O}, \lambda$, and of $\mathcal{O}, \mathcal{O}, T$, as described in Eqs. (A3) and (A9) of Appendix A.

As in Eqs. (2.25)-(2.27), the dumbbell two-point function of scalar operators $\mathcal{O}_{1}, \mathcal{O}_{2}$ with scaling dimensions $x_{1}, x_{2}$ such as $\varphi, \varphi$ or $\varphi, \varepsilon$ or $\epsilon, \epsilon$ follows from the two-point function in the film via

$\left\langle\mathcal{O}_{1}\left(\mathbf{r}_{1}\right) \mathcal{O}_{2}\left(\mathbf{r}_{2}\right)\right\rangle_{\mathrm{DB}}^{(i, j)}=b\left(\hat{\mathbf{r}}_{1}\right)^{x_{1}} b\left(\hat{\mathbf{r}}_{2}\right)^{x_{2}}\left\langle\mathcal{O}_{1}\left(\hat{\mathbf{r}}_{1}\right) \mathcal{O}_{2}\left(\hat{\mathbf{r}}_{2}\right)\right\rangle_{\text {film }}^{(i, j)}$. 
For $\mathcal{O}_{1}=\mathcal{O}_{2}=\mathcal{O}$ equal to $\varphi$ or $\epsilon$ we use Eq. (2.39) to calculate the film average on the right-hand side of Eq. (2.42) for small distance $\left|\hat{\mathbf{r}}_{1}-\hat{\mathbf{r}}_{2}\right|$ and check whether the resulting dumbbell average on the left-hand side agrees with the small dumbbell expansion.

First consider the $T$-contribution in Eq. (2.39). Using

$\sum_{\mu, \nu} s_{\mu} s_{\nu}\left\langle T_{\mu \nu}(\hat{\mathbf{r}})\right\rangle_{\text {film }}^{(i, j)}=(2 L)^{-d} \Delta_{i, j}\left[-\hat{\mathbf{r}}_{12}^{2}+d\left(\hat{z}_{1}-\hat{z}_{2}\right)^{2}\right]$,

we find from the transformation formulas (2.42), (2.25) and from comparing with the bulk three-point function (A9) of $\mathcal{O}, \mathcal{O}, T$ that

$$
\begin{aligned}
& \left.\left\langle\mathcal{O}\left(\mathbf{r}_{1}\right) \mathcal{O}\left(\mathbf{r}_{2}\right)\right\rangle_{\mathrm{DB}}^{(i, j)}\right|_{\text {from } T} \\
& \quad=(L / 2)^{d} \Delta_{i, j} \frac{d}{B_{T}}\left\langle\mathcal{O}\left(\mathbf{r}_{1}\right) \mathcal{O}\left(\mathbf{r}_{2}\right) T_{\|,\| \|}(0)\right\rangle_{\text {bulk }},
\end{aligned}
$$

which is consistent with the small dumbbell anisotropic operator (2.15). Here we have used Eq. (2.30) with $\mathcal{O}(\mathbf{r})$ replaced by $\mathcal{O}\left(\mathbf{r}_{1}\right) \mathcal{O}\left(\mathbf{r}_{2}\right)$.

Now consider the contribution from $\mathcal{D} \epsilon$ in Eq. (2.39). Expanding

$$
\begin{aligned}
& \mathcal{D}_{0, x_{\epsilon}}\langle\epsilon(\hat{\mathbf{r}})\rangle_{\text {film }}^{(i, j)}=(2 L)^{-x_{\epsilon}} B_{\epsilon}^{1 / 2}\left\{1+\frac{1}{8\left(x_{\epsilon}+1\right)}\right. \\
& \times\left[-\left(\frac{x_{\epsilon}}{2 x_{\epsilon}-d+2} \hat{\mathbf{r}}_{12}^{2}\right)\right. \\
& \left.\left.+\left(\hat{z}_{1}-\hat{z}_{2}\right)^{2}\right] \partial_{\hat{z}}^{2}\right\} \mathcal{E}_{i, j}(\hat{z} / L)
\end{aligned}
$$

with $\hat{z}=\left(\hat{z}_{1}+\hat{z}_{2}\right) / 2$ to second order in the small lengths $\hat{z}_{1}, \hat{z}_{2}$, the transformation formulas (2.42), (2.25) lead to

$$
\begin{aligned}
\left.\left\langle\mathcal{O}\left(\mathbf{r}_{1}\right) \mathcal{O}\left(\mathbf{r}_{2}\right)\right\rangle_{\mathrm{DB}}^{(i, j)}\right|_{\text {from } \mathcal{E} \epsilon}= & \left\langle\mathcal { O } ( \mathbf { r } _ { 1 } ) \mathcal { O } ( \mathbf { r } _ { 2 } ) \left[ a_{1}^{(i, j)} \boldsymbol{\epsilon}\right.\right. \\
& \left.\left.+a_{2}^{(i, j)} \Delta \epsilon+b_{1}^{(i, j)} \partial_{\|}^{2} \boldsymbol{\epsilon}\right]\right\rangle_{\text {bulk }},
\end{aligned}
$$

consistent with the small dumbbell operators (2.9). Here the bulk three-point function (A3) for $\left\langle\mathcal{O}_{1}\left(\mathbf{r}_{1}\right) \mathcal{O}_{2}\left(\mathbf{r}_{2}\right) \epsilon\left(\mathbf{r}_{\mathrm{DB}}\right)\right\rangle_{\text {bulk }}$ has been used. In the same way the $\lambda$ term in Eq. (2.39) leads to a contribution $\left.\left\langle\mathcal{O}\left(\mathbf{r}_{1}\right) \mathcal{O}\left(\mathbf{r}_{2}\right)\right\rangle_{\mathrm{DB}}^{(i, j)}\right|_{\text {from } \lambda}$, which equals $\left\langle\mathcal{O}\left(\mathbf{r}_{1}\right) \mathcal{O}\left(\mathbf{r}_{2}\right) a_{3}^{(i, j)} \lambda\right\rangle_{\text {bulk }}$.

Finally consider the mixed two-point function $\langle\varphi \epsilon\rangle_{\mathrm{DB}}^{i, j}$ for $(i, j)=(+,+)$ or $(-,+)$. Here we use the operator product expansion

$$
\varphi\left(\hat{\mathbf{r}}_{1}\right) \epsilon\left(\hat{\mathbf{r}}_{2}\right)=\frac{C_{\varphi \varphi \epsilon} / B_{\varphi}}{s^{x_{\epsilon}}} \mathcal{D}_{x_{\varphi}-x_{\epsilon}, x_{\varphi}}\left(\mathbf{s}, \partial_{\hat{\mathbf{r}}}\right) \varphi(\hat{\mathbf{r}})+\cdots
$$

with the short distance vector from Eq. (2.40). Since the two operators $\varphi, \epsilon$ have different scaling dimensions,

$$
\hat{\mathbf{r}}=\hat{\mathbf{r}}_{1}-\frac{x_{\epsilon}}{2 x_{\varphi}}\left(\hat{\mathbf{r}}_{1}-\hat{\mathbf{r}}_{2}\right), \quad \hat{z}=\hat{z}_{1}-\frac{x_{\epsilon}}{2 x_{\varphi}}\left(\hat{z}_{1}-\hat{z}_{2}\right)
$$

differs from $\hat{\mathbf{r}}$ in Eq. (2.41). Equations (A1) and (2.11) with $\mathcal{O}=\varphi$ imply

$$
\begin{aligned}
\mathcal{D}_{x_{\varphi}-x_{\epsilon}, x_{\varphi}}\langle\varphi(\hat{\mathbf{r}})\rangle_{\text {film }}^{(i, j)} & \\
= & (2 L)^{-x_{\varphi}} B_{\varphi}^{1 / 2}\left\{1+\frac{1}{8} \frac{x_{\epsilon}\left[2-\left(x_{\epsilon} / x_{\varphi}\right)\right]}{x_{\varphi}\left(x_{\varphi}+1\right)}\right. \\
& \left.\times\left[-\left(\frac{x_{\varphi}}{2 x_{\varphi}-d+2} \hat{\mathbf{r}}_{12}^{2}\right)+\left(\hat{z}_{1}-\hat{z}_{2}\right)^{2}\right] \partial_{\hat{z}}^{2}\right\} \mathcal{F}_{i, j}(\hat{z} / L) .
\end{aligned}
$$

For the $(+,+)$ dumbbell in Fig. 1(b) with $\mathcal{F}_{i, j}=\mathcal{F}_{++}$an even function of $\hat{z} / L$, the expansion of Eq. (2.49) to second order in the small quantities $\hat{z}_{1}, \hat{z}_{2}$, the transformation formulas (2.42), (2.25), and comparison with the bulk threepoint function $\langle\varphi \epsilon \varphi\rangle_{\text {bulk }}$ from Eq. (A3) lead to

$$
\begin{aligned}
\left\langle\varphi\left(\mathbf{r}_{1}\right) \epsilon\left(\mathbf{r}_{2}\right)\right\rangle_{\mathrm{DB}}^{(++)} \rightarrow & \left\langle\varphi ( \mathbf { r } _ { 1 } ) \epsilon ( \mathbf { r } _ { 2 } ) \left[\widetilde{a}_{1} \varphi+\widetilde{a}_{2} \Delta \varphi\right.\right. \\
& \left.\left.+\widetilde{b}_{1} \partial_{\|}^{2} \varphi\right]\right\rangle_{\text {bulk }},
\end{aligned}
$$

i.e., the left hand side is consistent with the small dumbbell operators in Eq. (2.18).

For the $(-+)$ dumbbell in Fig. 1(c) with $\mathcal{F}_{i, j}=\mathcal{F}_{-+}$an odd function of $\hat{z} / L$, we expand to first order in $\hat{z}_{1}, \hat{z}_{2}$ in Eq. (2.49) (the first term in curly brackets) and disregard the next corrections of third order. This amounts to neglecting contributions of order $L^{x_{\varphi}+3}$ to $\langle\varphi \epsilon\rangle_{\mathrm{DB}}^{-+}$and leads, via the transformation formulas and comparing with the bulk three-point function of $\varphi, \epsilon, \varphi$, to

$$
\left\langle\varphi\left(\mathbf{r}_{1}\right) \epsilon\left(\mathbf{r}_{2}\right)\right\rangle_{\mathrm{DB}}^{(-+)} \rightarrow\left\langle\varphi\left(\mathbf{r}_{1}\right) \epsilon\left(\mathbf{r}_{2}\right) \widetilde{c}_{1} \partial_{\|} \varphi\right\rangle_{\text {bulk }},
$$

consistent with the small dumbbell expansion for a $(-+)$ dumbbell containing the operator (2.19).

\section{RESULTS NEAR FOUR AND IN TWO DIMENSIONS}

Here we derive density profiles and density two-point functions in the presence of a dumbbell for $d$ near 4 and $d$ $=2$. While the results for the profiles are already sufficient to determine the small particle amplitudes, the two-point results allow for interesting consistency checks of the proposed operator expansions for small dumbbells and the related operator product expansions (2.39) and (2.47) for film geometries.

For spatial dimensions close to four the Ising model representing the critical fluid can be described in terms of scalar $\Phi^{4}$ theory, with the order parameter $\varphi$ and energy density $\epsilon$ proportional to $\Phi$ and $-\Phi^{2}$, respectively. Both $\langle\Phi\rangle_{\text {bulk }}$ and $\left\langle-\Phi^{2}\right\rangle_{\text {bulk }}$ vanish at the critical point in the bulk, since we use dimensional regularization and the $\varepsilon$ expansion. In two spatial dimensions results for profiles and multipoint averages for the Ising model at the critical point in strip geometry are known from conformal invariance methods. Note that the energy density profile $\langle\epsilon\rangle$ at the critical point, which is defined to vanish in the bulk, is negative and positive near a normal $(+$ or -$)$ surface and a neutral "ordinary" surface, respectively, where the Ising spin disorder is decreased and increased compared with the bulk.

\section{A. Symmetry breaking dumbbells near four dimensions}

As the spatial dimension $d=4-\varepsilon$ approaches $4, x_{\varphi}$ $\rightarrow 1, x_{\epsilon} \rightarrow 2, x_{\lambda} \rightarrow 4$, and the behavior of the critical fluid sur- 
rounding one of the two dumbbells in Figs. 1(b) and 1(c) with symmetry-breaking surfaces is determined by mean field expressions of the $\Phi^{4}$ theory. In the large-distance limit with dumbbell size $L$ much larger than microscopic lengths, the coupling constant can be taken at its fixed point value.

\section{Density profiles}

For the scaling functions $\mathcal{F}$ of the order parameter profile in films ${ }^{8,26}$ with $(i, j)=(+,+)$ or $(-,+)$ boundary conditions, one finds

$$
\mathcal{F}_{i, j}(y)=A F_{i, j}(y), \quad A=3 \varepsilon^{-1 / 2}
$$

for $\varepsilon \rightarrow 0$, with $y=\hat{z} / L$ as in Eq. (2.11) and

$$
F_{++}=\frac{\sqrt{2} K}{\operatorname{cn}(y K)}, \quad F_{-+}=2 K \frac{\operatorname{sn}(y K) \operatorname{dn}(y K)}{\operatorname{cn}(y K)} .
$$

Here sn, cn, and dn are Jacobian elliptic functions with the parameter $m=k^{2}=1 / 2$, and $K=K(m=1 / 2)=1.854$ is the corresponding complete elliptic integral. While

$$
F_{++} \rightarrow \sqrt{2} K+K^{3} y^{2} / \sqrt{2}+O\left(y^{4}\right), F_{-+} \rightarrow 2 K^{2} y+O\left(y^{5}\right)
$$

for small $y$, for $y \nearrow 1$ the functions $F_{++}$and $F_{-+}$tend to $2 /(1-y)$, consistent with the well known $\varepsilon$-expansion

$$
\mathcal{A}_{\varphi}^{(+)}=A[1+O(\varepsilon)]
$$

of the universal amplitude $\mathcal{A}_{\varphi}^{(+)}$of the order parameter profile in the half space with + boundary condition, as introduced below Eq. (2.13).

The functions $F=F_{++}, F_{-+}$satisfy the mean field equation

$$
2 F^{\prime \prime}=F^{3},
$$

with the prime denoting a derivative with respect to the argument. The combination $\left(F^{\prime}\right)^{2}-F^{4} / 4$ is independent of $y$ and determines the amplitude

$$
\Delta_{i, j} \rightarrow \frac{3}{2 \pi^{2}} \frac{1}{\varepsilon}\left(\left(F^{\prime}\right)^{2}-\frac{1}{4} F^{4}\right)=\frac{3}{2 \pi^{2}} \frac{1}{\varepsilon}[-1,4] K^{4}
$$

of the stress average in Eq. (2.16) for an $(i, j)=[(+,+)$, $(-,+)]$ film as $d / 4$. Since the universal amplitude $B_{T}$ in Eq. (2.15) for $d / 4$ approaches its value $1 /\left(3 \pi^{4}\right)$ in the Gaussian model, see Eq. (3.35) below, the stress tensor amplitude $b_{2}$ in Eq. (2.15) is given by

$$
b_{2}^{(i, j)} / L^{d}=\frac{9 \pi^{2}}{8} K^{4}[-1,4] \frac{1}{\varepsilon}
$$

for an $(i, j)=[(+,+),(-,+)]$ dumbbell.

The scaling functions of the film profiles of the energy density $\epsilon$ and the operator $\lambda$,

$$
\mathcal{E}_{i, j}=-\frac{A^{2}}{\sqrt{2}}\left(F_{i, j}\right)^{2}, \quad \mathcal{L}_{i, j}=\frac{A^{4}}{\sqrt{24}}\left(F_{i, j}\right)^{4}
$$

are determined by the second and fourth powers of the order parameter profile. Equation (3.8) is consistent with the $\varepsilon$-expansions

$$
\mathcal{A}_{\epsilon}^{(+)}=-\frac{A^{2}}{\sqrt{2}}[1+O(\varepsilon)], \quad \mathcal{A}_{\lambda}^{(+)}=\frac{A^{4}}{\sqrt{24}}[1+O(\varepsilon)]
$$

of the universal half-space amplitudes of $\epsilon$ and $\lambda$. While the leading contribution (3.8) to the profile $\mathcal{E}_{-,+}$vanishes on the midplane $y=0$ of the film, we expect that higher-order $\varepsilon$ contributions lead to a positive $\mathcal{E}_{-,+}(0)$, see the discussion at the end of Sec. IIIC 1.

The film profile scaling functions $\mathcal{P}=\mathcal{F}, \mathcal{E}, \mathcal{L}$ in Eqs. (3.1) and (3.2) and Eqs. (3.8) and (3.9) determine, via Eqs. (2.11) and (2.28), the profiles $\langle\mathcal{O}\rangle_{\mathrm{DB}}$ for $\mathcal{O}=\varphi, \epsilon, \lambda$ in the presence of a dumbbell and, via Eqs. (2.9), (2.14), (2.18), and (2.19), the amplitudes in the small particle expansion (2.1) and (2.2) for the dumbbell. For a $(+,+)$ dumbbell

$$
\begin{aligned}
& a_{1} B_{\epsilon}^{1 / 2} /\left(\mathcal{A}_{\epsilon}^{(+)} L^{x} \epsilon\right)=K^{2} / 2, \\
& \left(a_{2}, b_{1}\right) B_{\epsilon}^{1 / 2} /\left(\mathcal{A}_{\epsilon}^{(+)} L^{x_{\epsilon}+2}\right)=\left(K^{4} / 96, K^{4} / 48\right), \\
& a_{\lambda} B_{\lambda}^{1 / 2} /\left(\mathcal{A}_{\lambda}^{(+)} L^{x_{\lambda}}\right)=K^{4} / 4,
\end{aligned}
$$

for the amplitudes of operators even in the Ising up-down symmetry and

$$
\begin{aligned}
& \widetilde{a}_{1} B_{\varphi}^{1 / 2} /\left(\mathcal{A}_{\varphi}^{(+)} L^{x_{\varphi}}\right)=K / \sqrt{2}, \\
& \widetilde{a}_{2} B_{\varphi}^{1 / 2} \eta /\left(\mathcal{A}_{\varphi}^{(+)} L^{x_{\varphi}+2}\right)=K^{3} /(16 \sqrt{2}), \\
& \widetilde{b}_{1} B_{\varphi}^{1 / 2} /\left(\mathcal{A}_{\varphi}^{(+)} L^{x_{\varphi}+2}\right)=K^{3} /(16 \sqrt{2}),
\end{aligned}
$$

for the amplitudes of the odd operators. In Eq. (3.14) $\eta$ $=2 x_{\varphi}+2-d$ denotes, as usual, the anomalous part of twice the scaling dimension of the order parameter.

For a $(-, \quad+)$ dumbbell, $a_{1} B_{\epsilon}^{1 / 2} /\left(\mathcal{A}_{\epsilon}^{(+)} L^{x} \epsilon\right)$ and $a_{\lambda} B_{\lambda}^{1 / 2} /\left(\mathcal{A}_{\lambda}^{(+)} L^{x_{\lambda}}\right)$ tend to zero for $\varepsilon \searrow 0$,

$$
\left(a_{2}, b_{1}\right)=2\left(a_{2}^{(+,+)}, b_{1}^{(+,+)}\right)
$$

is twice as large as the above expressions for a $(+,+)$ dumbbell, and the amplitude of the odd operator in Eq. (2.8) is given by

$$
\widetilde{c}_{1} B_{\varphi}^{1 / 2} /\left(\mathcal{A}_{\varphi}^{(+)} L^{x_{\varphi}+1}\right)=K^{2} / 2 .
$$

\section{Two-point functions}

Here we consider two-point averages of the order parameter and energy densities. For $\varepsilon \rightarrow 0$ they approach the mean field results, which for the film geometry read

$$
\begin{aligned}
&\left\langle\varphi\left(\hat{\mathbf{r}}_{1}\right) \varphi\left(\hat{\mathbf{r}}_{2}\right)\right\rangle_{\mathrm{film}} / B_{\varphi}=(2 L)^{-2} A^{2} F\left(y_{1}\right) F\left(y_{2}\right), \\
&\left\langle\varphi\left(\hat{\mathbf{r}}_{1}\right) \epsilon\left(\hat{\mathbf{r}}_{2}\right)\right\rangle_{\mathrm{film}} /\left(B_{\varphi} B_{\epsilon}\right)^{1 / 2}=-(2 L)^{-3}\left(A^{3} / \sqrt{2}\right) F\left(y_{1}\right) \\
& \times\left[F\left(y_{2}\right)\right]^{2}, \\
&\left\langle\epsilon\left(\hat{\mathbf{r}}_{1}\right) \epsilon\left(\hat{\mathbf{r}}_{2}\right)\right\rangle_{\mathrm{film}} / B_{\epsilon}=(2 L)^{-4}\left(A^{4} / 2\right)\left[F\left(y_{1}\right) F\left(y_{2}\right)\right]^{2}
\end{aligned}
$$

with $A$ from Eq. (3.1) and the functions $F=F_{++}$or $F_{-+}$ from Eq. (3.2) for a film with $(+,+)$ or $(-,+)$ boundary conditions. Two-point averages $\left\langle\mathcal{O}_{1} \mathcal{O}_{2}\right\rangle_{\mathrm{DB}}$ in the presence of a $(+,+)$ or $(-,+)$ dumbbell follow from Eqs. (3.18)-(3.20) on using the transformation formulas (2.25), (2.27), and (2.42). For example, 


$$
\left\langle\varphi\left(\mathbf{r}_{1}\right) \varphi\left(\mathbf{r}_{2}\right)\right\rangle_{\mathrm{DB}} / B_{\varphi}=\frac{L^{2}}{4 r_{1}^{2} r_{2}^{2}} A^{2} F\left(\frac{r_{1 \|}}{r_{1}^{2}} L\right) F\left(\frac{r_{2 \|}}{r_{2}^{2}} L\right) .
$$

It is instructive to check that the film results (3.18)(3.20) of leading order in $\varepsilon$ agree, for $\hat{\mathbf{r}}_{1} \rightarrow \hat{\mathbf{r}}_{2}$, with the operator product expansions (2.39) and (2.47) and that the corresponding dumbbell results, such as Eq. (3.21) for dumbbell size $L \ll r_{1}, r_{2}$, are consistent with the small particle expansion, i.e., with the contributions (2.44), (2.46), (2.50), (2.51), and the $\lambda$ contribution below Eq. (2.46). For example, the four operators $\left(\Delta, \partial_{\|}^{2}\right) \epsilon, T_{\|,\|}$, and $\lambda$ cooperate in an interesting way to reproduce the mean field results of second order in $\hat{\mathbf{r}}_{12}$ in Eq. (3.18) or (3.22) for the film, and of fourth order in $L$ in Eq. (3.21) for the dumbbell, as both their scaling dimensions $x_{\epsilon}+2, d$, and $d+\omega$ approach 4 for $\varepsilon \rightarrow 0$. In higher order in $\varepsilon$, the operator expansions help disentangle contributions which belong to different powers of the small distance or particle size.

First consider the film average of two order parameters in Eq. (3.18) with the expansion

$$
\begin{aligned}
\left\langle\varphi\left(\hat{\mathbf{r}}_{1}\right) \varphi\left(\hat{\mathbf{r}}_{2}\right)\right\rangle_{\text {film }} / B_{\varphi} & \rightarrow\left(\frac{A}{2 L}\right)^{2}\left\{[F(y)]^{2}+\left(\frac{y_{1}-y_{2}}{2}\right)^{2}\right. \\
& \left.\times\left\{-\left[F^{\prime}(y)\right]^{2}+F(y) F^{\prime \prime}(y)\right\}\right\}
\end{aligned}
$$

about an arbitrary interior point $(2.41)$ of the $(+,+)$ or $(-$, + ) film, with

$$
y=\left(y_{1}+y_{2}\right) / 2 .
$$

In order to compare Eq. (3.22) with the film average of Eq. (2.39) with $\mathcal{O}=\varphi$, we need the results

$$
\frac{C_{\varphi \varphi \epsilon}}{B_{\varphi} B_{\epsilon}^{1 / 2}}=-\sqrt{2}, \frac{C_{\varphi \varphi T}}{B_{\varphi}}=-\frac{2}{3 \pi^{2}}, \frac{C_{\varphi \varphi \lambda}}{B_{\varphi} B_{\lambda}^{1 / 2}}=\frac{\varepsilon}{3 \sqrt{6}}
$$

to leading order in $\varepsilon$ for the universal amplitude ratios of bulk three-point functions containing two order parameter fields, with the nonuniversal amplitudes $B$ and $C$ defined in Eqs. (2.10) and (A3), (A9). While the results for $C_{\varphi \varphi \epsilon}$ and $C_{\varphi \varphi T}$ coincide with those in the Gaussian model without the $\Phi^{4}$ interaction, the result for $C_{\varphi \varphi \lambda}$ requires a higher order loop calculation considered in Appendix B.

The leading short distance contribution to the film average of the operator product expression (2.39) with $\mathcal{O}=\varphi$ is of order $s^{-2 x} \varphi$. However, this power is accompanied by an amplitude of order $\varepsilon^{0}$ and only shows up in the correction to the result (3.22), which is of order $\varepsilon^{-1}$. This is confirmed by the calculation in section 2 of Appendix B in next to leading order in $\varepsilon$ of the two-point average $\langle\varphi(1) \varphi(2)\rangle_{\mathrm{hs}}^{(+)}$for the case of a half space with surface magnetic field $+\infty$.

The next to leading short distance contribution is of order $s^{-2 x_{\varphi}+x_{\epsilon}}$ and comes from the first term of the differential operator $\mathcal{D}_{0, x_{\epsilon}}$ in Eq. (A1). For $\varepsilon \rightarrow 0$ this contribution arising from Eq. (2.39) reproduces the first term in curly brackets of the result to leading order in $\varepsilon$ on the right hand side of Eq. (3.22). Here one uses Eqs. (2.11) and (3.8) to calculate $\left\langle\epsilon(\hat{\mathbf{r}}) / B_{\epsilon}^{1 / 2}\right\rangle_{\text {film }}$, Eq. (3.24) for the amplitude $C_{\varphi \varphi \epsilon}$, and the fact that the power exponent $-2 x_{\varphi}+x_{\epsilon}$ tends to zero for $\varepsilon$ $\rightarrow 0$.

The product expansion (2.39) predicts three next to next to leading contributions $\kappa_{1}, \kappa_{2}, \kappa_{3}$ to $\left\langle\varphi\left(\hat{\mathbf{r}}_{1}\right) \varphi\left(\hat{\mathbf{r}}_{2}\right)\right\rangle_{\text {film }} / B_{\varphi}$, which are of order $s^{-2 x_{\varphi}+x_{\epsilon}+2}, s^{-2 x_{\varphi}+d}, s^{-2 x_{\varphi}+x_{\lambda}}$, and correspond to second derivatives of the energy density $\epsilon$, the stress tensor, and the operator $\lambda$. For $\varepsilon \rightarrow 0$ all three are of second order in the small distance vector $\mathbf{s}=\hat{\mathbf{r}}_{12}$ and have the form

$$
\begin{aligned}
& \kappa_{1} \rightarrow \frac{A^{2}}{(2 L)^{4}}\left[s^{2}-\left(\hat{z}_{1}-\hat{z}_{2}\right)^{2}\right]\left(-\frac{1}{3}\left(F^{\prime}\right)^{2}-\frac{1}{6} F^{4}\right), \\
& \kappa_{2} \rightarrow \frac{A^{2}}{(2 L)^{4}}\left[s^{2}-4\left(\hat{z}_{1}-\hat{z}_{2}\right)^{2}\right]\left(\frac{1}{3}\left(F^{\prime}\right)^{2}-\frac{1}{12} F^{4}\right), \\
& \kappa_{3} \rightarrow \frac{A^{2}}{(2 L)^{4}} s^{2} \frac{1}{4} F^{4} .
\end{aligned}
$$

Besides Eq. (2.39), the values of $A$ and $B_{T}$ in Eq. (3.1) and below Eq. (3.6), and the three-point amplitudes (3.24), we have used Eqs. (2.45) with Eq. (3.8), Eq. (2.43) with Eq. (3.6), and Eq. (2.11) with Eq. (3.8) to derive Eqs. (3.25), (3.26), and (3.27). In the sum $\kappa_{1}+\kappa_{2}+\kappa_{3}$, the $s^{2}$ contributions cancel, and the $\left(\hat{z}_{1}-\hat{z}_{2}\right)^{2}$ contributions reproduce the $\left(y_{1}-y_{2}\right)^{2}$ term of the result (3.22) to leading order in $\varepsilon$, as expected. Here Eq. (3.5) has been used to express $F F^{\prime \prime}$ in Eq. (3.22) in terms of $F^{4}$.

A similar cancellation occurs on summing the three contributions

$$
\begin{aligned}
\left\langle\varphi\left(\mathbf{r}_{1}\right) \varphi\left(\mathbf{r}_{2}\right)\left[a_{2} \Delta \epsilon+b_{1} \partial_{\|}^{2} \boldsymbol{\epsilon} ; b_{2} T_{\|,\|} ; a_{3} \lambda\right]\right\rangle_{\text {bulk }} / B_{\varphi} \\
=\left(\frac{L}{2}\right)^{4} \frac{1}{\left(r_{1} r_{2}\right)^{2}} \frac{3}{\varepsilon}\left[\left(4 U_{+}+4 U_{12}-I\right)\left(F^{\prime 2}+\frac{1}{2} F^{4}\right) ;\right. \\
\left.\left(-4 U_{+}+8 U_{12}+I\right)\left(F^{\prime 2}-\frac{1}{4} F^{4}\right) ; \frac{3}{4} I F^{4}\right]
\end{aligned}
$$

of order $L^{x}{ }^{+2}, L^{d}$, and $L^{x_{\lambda}}$ in the small particle expansion of the order parameter two-point function $\left\langle\varphi\left(\mathbf{r}_{1}\right) \varphi\left(\mathbf{r}_{2}\right)\right\rangle_{\mathrm{DB}} / B_{\varphi}$ in the presence of a small $(+,+)$ or $(-$, $+)$ dumbbell. These expressions follow from Eqs. (A5) with Eqs. (3.8) and (A3), Eq. (2.44) with Eqs. (A11) and (3.6), and Eq. (2.14) with Eq. (3.8) on inserting the values of the three-point amplitudes from Eq. (3.24) and of $A$ and $B_{T}$ from Eq. (3.1) and below Eq. (3.6). In Eq. (3.28) $I, U_{+}$, and $U_{12}$ are from Eq. (A8), and $F \equiv F(0), F^{\prime} \equiv F^{\prime}(0)$, and $F^{4}$ $\equiv[F(0)]^{4}$. As expected, the isotropic contribution $I$ drops out of the sum

$$
12 U_{12} F^{\prime 2}+3 U_{+} F^{4}
$$

of the three terms in square brackets in Eq. (3.28) and the sum of the three contributions in Eq. (3.28) reproduces the $L^{4}$ term of the result to leading order in $\varepsilon$,

$$
\begin{aligned}
& \left\langle\varphi\left(\mathbf{r}_{1}\right) \varphi\left(\mathbf{r}_{2}\right)\right\rangle_{\mathrm{DB}} / B_{\varphi} \\
& \quad=\frac{L^{2}}{4 r_{1}^{2} r_{2}^{2}} A^{2}\left\{F^{2}+\frac{1}{2} L^{2} U_{+} F F^{\prime \prime}, L^{2} U_{12} F^{\prime 2}\right\}
\end{aligned}
$$


for a $\{(+,+),(-,+)\}$ dumbbell, which follows from Eq. (3.21).

Next we consider the mixed two-point average $\left\langle\varphi\left(\hat{\mathbf{r}}_{1}\right) \epsilon\left(\hat{\mathbf{r}}_{2}\right)\right\rangle_{\text {film }}$ and compare the $\hat{\mathbf{r}}_{1,2} \rightarrow 0$ behavior of the result (3.19) to leading order $\varepsilon \rightarrow 0$ for an arbitrary interior point $\hat{\mathbf{r}}$ of the $(+,+)$ or $(-,+)$ film with the film average of the operator product expansion (2.47), which applies in any spatial dimension. One finds that the leading behavior $-(2 L)^{-3}\left(A^{3} / \sqrt{2}\right)[F(y)]^{3}$ of order $s^{0} \varepsilon^{-3 / 2}$ in Eq. (3.19) is reproduced by the film average of the term of order $s^{-x_{\epsilon}+2}$ in Eq. (2.47). Besides the three-point amplitude $C_{\varphi \varphi \epsilon}$ in Eq. (3.24), the relations (3.1) and (3.4) between $\mathcal{F}, F$, and $\varepsilon$, and Eq. (3.5) between $F^{\prime \prime}$ and $F^{3}$, one uses that the combinations $2-\left(x_{\epsilon} / x_{\varphi}\right)$ and $2 x_{\varphi}-d+2=\eta$ of exponents in Eq. (2.49) tend to $-\varepsilon / 3$ and $\varepsilon^{2} / 54$, respectively, as $\varepsilon \rightarrow 0$. Note that the $\varepsilon \rightarrow 0$ limit (3.19) for $\langle\varphi \epsilon\rangle_{\text {film }}$ is of order $\varepsilon^{-3 / 2}$ and misses the leading short distance behavior $\propto s^{-x} \epsilon$, given by Eqs. (2.47) and (2.49), which has an amplitude of order $\varepsilon^{-1 / 2}$.

There are similar results for a small $(+,+)$ dumbbell. The normalized mixed product average $\left\langle\varphi\left(\mathbf{r}_{1}\right) \epsilon\left(\mathbf{r}_{2}\right)\right\rangle_{\mathrm{DB}}^{(+,+)} /\left(B_{\varphi} B_{\epsilon}\right)^{1 / 2}$ due to Eqs. (2.42) and (3.19) has a leading $\varepsilon \rightarrow 0$ behavior of order $\varepsilon^{-3 / 2}$, which for $\mathbf{r}_{12}$ $\rightarrow 0$ tends to the isotropic expression $-\left[L^{3} /\left(8 r_{1}^{2} r_{2}^{4}\right)\right]$ $\times\left(A^{3} / \sqrt{2}\right)\left[F_{++}(0)\right]^{3}$. On inserting $\mathcal{F}_{++}^{\prime \prime}(0)$ from Eq. (3.1) in Eq. (A7), this expression is reproduced by the $I$ term, which is the only term of order $\varepsilon^{-3 / 2}$ in the small dumbbell operator expansion (2.50). The leading small dumbbell behavior of order $L^{x_{\varphi}}$ which arises from $\widetilde{a}_{1}$ in (2.50) is of order $\varepsilon^{-1 / 2}$ and not contained in the leading $\varepsilon \rightarrow 0$ expression.

For a $(-,+)$ dumbbell the leading $\varepsilon \rightarrow 0$ result for the mixed product average is of order $L^{6}$, i.e., it does not contain the leading small dumbbell behavior of order $L^{x_{\varphi}+1}$ given by Eq. (2.51) and corresponds to operators with higher scaling dimensions.

Finally consider two-point averages of the energy density. Only the $\lambda$ term in the product expansion (2.39) for $\epsilon \epsilon$ or in the expressions (2.9), (2.14), and (2.15) of even dumbbell operators leads to a contribution to $\langle\epsilon \epsilon\rangle_{\text {film }}$ or $\langle\epsilon \epsilon\rangle_{\mathrm{DB}}$ of the same order $\varepsilon^{-2}$ as the "direct" results given by Eqs. (3.20) and (2.42). Using that the universal three-point amplitude $C_{\epsilon \epsilon \lambda} /\left(B_{\epsilon} B_{\lambda}^{1 / 2}\right)$ tends to $\sqrt{6}$ for $\varepsilon \rightarrow 0$, one confirms that the $\lambda$ term in the expansion (2.39) about an arbitrary interior point of the film reproduces the value $(2 L)^{-4}\left(A^{4} / 2\right)$ $\times[F(y)]^{4}$ of Eq. (3.20) for $\hat{\mathbf{r}}_{12} \rightarrow 0$, and the dumbbell operator (2.14) reproduces the corresponding leading order in $\varepsilon$ result $\left[L^{4} /\left(16 r_{1}^{4} r_{2}^{4}\right)\right]\left(A^{2} / 2\right)\left[F_{++}(0)\right]^{4}$ of the normalized average $\left\langle\epsilon\left(\mathbf{r}_{1}\right) \epsilon\left(\mathbf{r}_{2}\right)\right\rangle_{\mathrm{DB}} / B_{\epsilon}$ in the presence of a $(+,+)$ dumbbell. Again, for a $(-,+)$ dumbbell the leading order $\varepsilon \rightarrow 0$ result is of order $L^{8}$ which corresponds to operators with higher scaling dimensions.

\section{B. Neutral (ordinary) dumbbell near four dimensions and results for the Gaussian model}

Consider a dumbbell which preserves the order parameter symmetry of the embedding critical fluid with the order parameter vanishing on the particle surface ("ordinary" surface with Dirichlet boundary condition ${ }^{5}$ ). In this case only the operators $\epsilon, \Delta \epsilon, \lambda, \ldots$ and $\partial_{\|}^{2} \epsilon, T_{\|,\|}, \ldots$ which are even in the Ising up-down symmetry show up in the small particle expansion, and for $d \rightarrow 4$ their weights coincide with those in the Gaussian model with a bulk Hamiltonian given by Eq. (B1) with $u=0$. Apart from representing a neutral particle in a critical binary fluid for $d \rightarrow 4$, a particle with 'ordinary' surface in the Gaussian model is also of interest for $2<d$ $<4$ since it describes a particle interacting with an ideal (random-walk-like) nonadsorbing polymer chain, see, e.g., Ref. 16 where a particle of ellipsoidal shape is considered.

For the small dumbbell expansion in the Gaussian model with $2<d<4$, we find the leading contribution

$$
a_{1} \epsilon=(L / 2)^{d-2} \mathcal{E}(0)\left(-\Phi^{2}\right) /\left(\sqrt{2} \widetilde{S}_{d}\right)
$$

from the energy density $\epsilon \propto-\Phi^{2}$, see Eq. (2.9), where

$$
\mathcal{E}(0)=\sqrt{2}\left(1-2^{3-d}\right) \zeta(d-2) .
$$

Here $\zeta$ is the Riemann zeta function, and

$$
\widetilde{S}_{d}=\Gamma((d-2) / 2) /\left(4 \pi^{d / 2}\right)
$$

is the amplitude in the Gaussian bulk propagator $\langle\Phi(\mathbf{r}) \Phi(0)\rangle_{\text {bulk }}=\widetilde{S}_{d} r^{2-d}$. The universal quantity $\mathcal{E}(0)$ equals $\pi^{2} /(6 \sqrt{2})=1.163$ and $\sqrt{2} \ln 2=0.980$, respectively, in $d=4$ and $d=3$. While the leading contribution has a size exponent $d-2$, the three contributions

$$
\begin{aligned}
\left\{a_{2} \Delta \epsilon\right. & \left.+b_{1} \partial_{\|}^{2} \epsilon ; b_{2} T_{\|,\|}\right\} \\
= & -(L / 2)^{d} \zeta(d)\left[2(d-2) \widetilde{S}_{d}\right]^{-1}\left\{\left(1-2^{-d}\right)\right. \\
& \left.\times\left[\Delta \Phi^{2}+(d-2) \partial_{\|}^{2} \Phi^{2}\right] ; 2^{-d+2}(d-1) T_{\|,\|}\right\}
\end{aligned}
$$

from the isotropic operator $\Delta \epsilon$ and the two leading anisotropic operators $\partial_{\|}^{2} \epsilon$ and $T_{\|,\|}$have a size exponent $d$. Equation (3.34) has been obtained from Eq. (2.9), Eq. (2.15), and the forms

$$
\begin{aligned}
& \mathcal{E}^{\prime \prime}(0)=\sqrt{2}\left(1-2^{-d}\right)(d-1)(d-2) \zeta(d), \\
& \Delta_{\text {ord,ord }}=-2^{-d} \pi^{-d / 2} \Gamma(d / 2) \zeta(d), \\
& B_{T}=\widetilde{S}_{d}^{2}(d-2)^{2} d /(d-1)
\end{aligned}
$$

of $\mathcal{E}^{\prime \prime}, \Delta_{\text {ord,ord }}, B_{T}$ in Eqs. (2.9), (2.15), and (2.17) for the Gaussian model at the critical point, in which

$$
T_{\|,\|}=\left(\partial_{\|} \Phi\right)^{2}-\frac{1}{2}(\nabla \Phi)^{2}-\frac{d-2}{4(d-1)}\left(\partial_{\|}^{2}-\Delta\right) \Phi^{2} .
$$

For later use we note the scaling function in the film

$$
\begin{aligned}
\mathcal{E}(y)= & 2^{(3 / 2)-d}[\zeta(d-2,(1-y) / 2)+\zeta(d-2,(1+y) / 2) \\
& -2 \zeta(d-2)]
\end{aligned}
$$

for arbitrary $y=\hat{z} / L$ in between -1 and +1 and the value $\mathcal{A}_{\epsilon}^{\text {(ord) }}=2^{(3 / 2)-d}$ of the half space amplitude. Note that $\mathcal{E}$ and $\mathcal{E}^{\prime \prime}(0)$ are positive for $2<d<4$, while $\Delta_{\text {ord,ord }}$ is negative. Finally the contribution

$$
a_{3} \lambda=(L / 2)^{2(d-2)} \mathcal{L}(0) \Phi^{4} /\left(2 \sqrt{6} \widetilde{S}_{d}^{2}\right)
$$

from the operator $\lambda \propto \Phi^{4}$ with $\lambda / B_{\lambda}^{1 / 2}=\Phi^{4} /\left(2 \sqrt{6} \widetilde{S}_{d}^{2}\right)$ and

$$
\mathcal{L}(0)=\sqrt{3 / 2}[\mathcal{E}(0)]^{2}
$$

has size dimension $2(d-2)$. 
The above expressions for $a_{1}, a_{2}, a_{3}, b_{1}, b_{2}$ not only describe the density profiles but also multipoint averages in presence of the dumbbell. We explicitly check this for the two-point averages $\langle\varphi \varphi\rangle_{\mathrm{DB}}$ and $\langle\epsilon \epsilon\rangle_{\mathrm{DB}}$ in Appendix $\mathrm{C}$ by confirming the predictions of the product expansion (2.39) with $\mathcal{O}=\varphi$ and $\epsilon$ for the short distance behavior of $\langle\varphi \varphi\rangle_{\text {film }}$ and $\langle\epsilon \epsilon\rangle_{\text {film }}$ about an arbitrary interior point of the film. There we also check that in the four-point function $\langle\varphi \varphi \varphi \varphi\rangle_{\mathrm{DB}}$, the contribution of order $L^{2(d-2)}$ in the small particle expansion is reproduced by the operator $a_{3} \lambda$ in Eq. (3.38).

It is interesting to compare the anisotropic effects of a dumbbell with that of other uniaxial anisotropic particles such as an ellipsoid (E) of revolution with major axis (largest distance between two surface points) $l$, minor axis $s$, and the interfocal distance $2 f=\sqrt{l^{2}-s^{2}}$. For the leading anisotropic behavior of the ellipsoid, we use the results of Ref. 16 in terms of the amplitudes $B_{\mathrm{VI}}, B_{\mathrm{VII}}$, where

$$
\begin{aligned}
b_{1, \mathrm{E}} \partial_{\|}^{2} \epsilon= & -\frac{f^{d}}{4}\left\{B_{\mathrm{VII}}+\left(B_{\mathrm{VI}}-B_{\mathrm{VII}}\right)(d-2) /\right. \\
& {[2(d-1)]\} \partial_{\|}^{2} \Phi^{2}, } \\
b_{2, \mathrm{E}} T_{\|,\|}= & -\frac{f^{d}}{2}\left(B_{\mathrm{VI}}-B_{\mathrm{VII}}\right) T_{\|,\|}
\end{aligned}
$$

with

$$
\begin{aligned}
& b_{1, \mathrm{E}} \partial_{\|}^{2} \boldsymbol{\epsilon}+b_{2, \mathrm{E}} T_{\|,\|} \\
& \quad=-f^{d}\left[B_{\mathrm{VI}}\left(\partial_{\|} \Phi\right)^{2} / 2+B_{\mathrm{VII}} \Phi\left(\partial_{\|}^{2} \Phi\right) / 2+\text { iso }\right]
\end{aligned}
$$

as follows on using Eq. (3.36), and where 'iso' denotes isotropic operators. In particular, we consider in $d=3$ spatial dimensions a prolate ellipsoid CE that circumscribes the dumbbell DB in Fig. 1, touches it at the highest and lowest points, and has the same curvature at these points, so that $l$ $=2 L, l / s=L / f=\sqrt{2}$. For the two particle shapes [DB; CE] we then obtain

$$
\begin{array}{r}
{\left[a_{1} ; a_{1, \mathrm{CE}}\right] \epsilon=-(L / \sqrt{2})[4 \pi \sqrt{2} \ln 2=12.3 ;} \\
4 \pi /(\operatorname{arcosh} \sqrt{2})=14.3] \Phi^{2} / 2,
\end{array}
$$

and

$$
\begin{aligned}
{\left[b_{1} ; b_{1, \mathrm{CE}}\right] \partial_{\|}^{2} \epsilon+\left[b_{2} ; b_{2, \mathrm{CE}}\right] T_{\|,\|} } \\
=-(L / \sqrt{2})^{3}\left\{[13.4 ; 8.31]\left(\partial_{\|} \Phi\right)^{2} / 2\right. \\
\left.\quad+[8.01 ; 4.76] \Phi\left(\partial_{\|}^{2} \Phi\right) / 2+\mathrm{iso}\right\} .
\end{aligned}
$$

Here we have used Eqs. (3.31)-(3.36) for the dumbbell with $(5,3) \pi \zeta(3) / \sqrt{2}=(13.4,8.01)$ and Eqs. (3.6) $-(3.10)$ in Ref. 16 for the ellipsoid with $\left(B_{\mathrm{VI}}, B_{\mathrm{VII}}\right)=(8.31,4.76)$.

Thus the leading isotropic and anisotropic perturbations of the critical system due to the DB are weaker and stronger, respectively, than for the circumscribed ellipsoid (CE). Indeed, the DB is smaller and more anisotropic than the CE. This shows up in the polymer-induced interaction between the particle and a planar wall. The result in Eqs. (3.56)(3.58) of Ref. 16 for the anisotropic interaction of an ellipsoidal particle can be extended to a particle with dumbbell shape by replacing $f$ with $L / \sqrt{2}$ and $\left(B_{\mathrm{VI}}, B_{\mathrm{VII}}\right)$ by the cor- responding numbers $(13.4,8.01)$ for the dumbbell. Equation (3.55) of Ref. 16 for the particle insertion free energy can be modified similarly by replacing $B_{\mathrm{I}}=14.3$ with 12.3 , see Eq. (3.42).

\section{Dumbbell in two dimensions}

\section{Density profiles and small particle amplitudes}

The two scaling functions $\mathcal{E}, \mathcal{F}$ of the density profiles $\langle\epsilon\rangle_{\text {film }},\langle\varphi\rangle_{\text {film }}$ in $d=2$ Ising strips with symmetric boundary conditions $(i, i)=($ ord,ord $)$ or $(+,+)$ are given by ${ }^{6}$

$$
\mathcal{P}_{i, i}(y)=\mathcal{A}_{\mathcal{O}}^{(i)}[\pi / \cos (\pi y / 2)]^{x_{\mathcal{O}}},
$$

with $-1<y<1$ and $\mathcal{P}=\mathcal{E}, \mathcal{F}$ for $\mathcal{O}=\epsilon, \varphi$, as in Eqs. (2.11) and (2.12). The bulk exponents $x_{\mathcal{O}}$ are $x_{\epsilon}=1, x_{\varphi}=1 / 8$, and the universal half plane amplitudes

$$
\mathcal{A}_{\epsilon}^{(\text {ord })}=-\mathcal{A}_{\epsilon}^{(+)}=1 / 2, \quad \mathcal{A}_{\varphi}^{(+)}=2^{1 / 8}
$$

follow from the two-point functions $\langle\epsilon \epsilon\rangle_{\mathrm{hs}}$ and $\langle\varphi \varphi\rangle_{\mathrm{hs}}$ in the half plane. ${ }^{23}\langle\varphi \varphi\rangle_{\mathrm{hs}}$ also determines the universal bulk three point amplitude

$$
C_{\varphi \varphi \epsilon} /\left(B_{\varphi} B_{\epsilon}^{1 / 2}\right)=-1 / 2
$$

including its sign, which we need for the discussion of the two-point functions $\left\langle\mathcal{O}_{1} \mathcal{O}_{2}\right\rangle_{\text {DB }}$ below. For the boundary conditions $(i, j)=(-,+)$, the even and odd functions

$$
\mathcal{E}_{-,+}(y)=\left[1-4 \cos ^{2}(\pi y / 2)\right] \mathcal{E}_{+,+}(y)
$$

and

$$
\mathcal{F}_{-,+}(y)=\sin (\pi y / 2) \mathcal{F}_{+,+}(y)
$$

are derived in Refs. 25(a,b). For a dumbbell composed of two touching circles in the plane, the small dumbbell expansion amplitudes then follow from Eqs. (2.9) and (2.18). For the symmetric dumbbells with $(i, j)=($ ord,ord) and $(+,+)$ boundary conditions in Figs. 1(a) and 1(b), one finds

$$
\begin{aligned}
& a_{1}^{(i, i)} B_{\epsilon}^{1 / 2} /\left(\mathcal{A}_{\epsilon}^{(i)} L^{x} \epsilon\right)=\pi / 2, \\
& \left(a_{2}^{(i, i)}, b_{1}^{(i, i)}\right) B_{\epsilon}^{1 / 2} /\left(\mathcal{A}_{\epsilon}^{(i)} L^{x_{\epsilon}+2}\right)=\left(\pi^{3} / 2^{7}\right)[(1 / 2), 1]
\end{aligned}
$$

for the amplitudes of the even isotropic and anisotropic operators $\epsilon, \Delta \epsilon$, and $\partial_{\|}^{2} \epsilon$. The amplitudes of the odd operators $\varphi, \Delta \varphi$, and $\partial_{\|}^{2} \varphi$ for a $(+,+)$ dumbbell are given by

$$
\begin{aligned}
& \widetilde{a}_{1}^{(+,+)} B_{\varphi}^{1 / 2} /\left(\mathcal{A}_{\varphi}^{(+)} L^{x}\right)=(\pi / 2)^{1 / 8}, \\
& \left(\widetilde{a}_{2}^{(+,+)}, \widetilde{b}_{1}^{(+,+)}\right) B_{\varphi}^{1 / 2} /\left(\mathcal{A}_{\varphi}^{(+)} L^{x_{\varphi}+2}\right)=(\pi / 2)^{17 / 8}(4,1) / 9 .
\end{aligned}
$$

For the dumbbell in Fig. 1(c) with boundary conditions $(i, j)=(-,+)$ the energy density amplitudes

$$
\begin{aligned}
& a_{1}^{(-,+)}=-3 a_{1}^{(+,+)}, \\
& \left(a_{2}^{(-,+)}, b_{1}^{(-,+)}\right)=5\left(a_{2}^{(+,+)}, b_{1}^{(+,+)}\right)
\end{aligned}
$$

are simply related to those of the $(+,+)$ dumbbell, and the amplitude of $\partial_{\|} \varphi$ is determined by

$$
\widetilde{c}_{1} B_{\varphi}^{1 / 2} /\left(\mathcal{A}_{\varphi}^{(+)} L^{x_{\varphi}+1}\right)=4(\pi / 2)^{9 / 8} .
$$

Finally the amplitudes of $T_{\|,\|}$,

$$
b_{2}^{(i, j)} / L^{d}=\left(\pi^{3} / 24\right)[-1,-1,23]
$$


for dumbbells with boundary conditions $(i, j)=[$ (ord,ord), $(+,+),(-,+)]$ follow from Eq. (2.15), since $\Delta_{i, j}$ $=(\pi / 48)[-1,-1,23]$ and $B_{T}=1 /\left(4 \pi^{2}\right)$ in the $d=2$ Ising model. ${ }^{23}$

Density profiles in the presence of a dumbbell follow from Eqs. (3.44)-(3.48) via the transformation formula (2.28). Note the interesting behavior of the energy density in the strip with $(-,+)$ boundary conditions. ${ }^{25}$ While the ordering near the - and + boundaries makes $\langle\epsilon\rangle_{\text {film }}$ negative for $2 / 3<|y|<1,\langle\epsilon\rangle_{\text {film }}$ becomes positive for $|y|<2 / 3$ due to a disorder arising from the two opposing surface ordering fields. One expects a similar change of sign for a $(-,+)$ film in dimension $d$ slightly below 4 if the calculation is pushed beyond the mean field result (3.2), (3.8), with $\langle\epsilon\rangle_{\text {film }}$ staying negative except for $y=0$ where it vanishes. For a $(-,+)$ dumbbell Eqs. (3.47) and (2.28) imply that $\langle\epsilon\rangle_{\mathrm{DB}}$ is negative in the region $L<r^{2} /\left|r_{\|}\right|<(3 / 2) L$ between the surfaces of the actual dumbbell with $(-,+)$ ordering fields in Fig. 1(c) and a "ghost" dumbbell which is larger by a factor $3 / 2$. In this region the order due to the field in the surface of the closer circle dominates, while in the region (3/2) $L<r^{2} /\left|r_{\|}\right|$outside the "ghost" dumbbell the disorder due to the two opposing surface fields dominates, and $\langle\epsilon\rangle_{\mathrm{DB}}$ is positive. Thus, at large distances the (ord,ord), $(+,+)$, and $(-,+)$ dumbbells in Fig. 1 induce disorder, order, and disorder of the Ising spins, respectively, and the energy density profile $\langle\epsilon\rangle_{\mathrm{DB}}$ and the amplitude $a_{1}$ of the operator $\epsilon$ in the small particle expansion are positive, negative, and positive.

\section{Two-point functions}

Explicit results for multipoint averages in Ising model strips can be found in Refs. 25(b)-25(d). Two-point averages in the presence of the dumbbells shown in Fig 1 then follow from the transformation formulas (2.25), (2.27), and (2.42) and are given in Eqs. (D1)-(D6) of Appendix D.

In order to check the small particle expansion, we expand the averages in the limit $L \ll r_{1}, r_{2}$ in which the quantity $C$ in Eq. (D7) becomes small and tends to

$$
C \rightarrow \Lambda_{1} \Lambda_{2} r_{12}^{2}\left[1+(\pi L)^{2} Q / 24+O\left(L^{4}\right)\right] .
$$

Here $\Lambda_{k}$ is from Eq. (D7), and

$$
Q=(I / 2)-U_{+}+2 U_{12}
$$

is the square bracket on the right hand side of Eq. (A11) for $d=2$, with $I, U_{+}$, and $U_{12}$ from Eq. (A8).

In the $d=2$ Ising model the three-point function $\langle\epsilon \epsilon \epsilon\rangle_{\text {bulk }}$ of the energy density vanishes, and of the small particle expansion terms (3.49)-(3.53), only the stress tensor contributes to $\langle\epsilon \epsilon\rangle_{\mathrm{DB}}^{(i, j)}$. Expanding Eqs. (D2) and (D5) for small particle size leads to

$$
\begin{aligned}
\left\langle\epsilon\left(\mathbf{r}_{1}\right) \epsilon\left(\mathbf{r}_{2}\right)\right\rangle_{\mathrm{DB}}^{(i, j)} / B_{\epsilon}= & r_{12}^{-2}\left\{1+[-1,-1,23](\pi L)^{2} Q / 24\right. \\
& \left.+O\left(L^{4}\right)\right\}
\end{aligned}
$$

for $(i, j)=[($ ord,ord $),(+,+),(-,+)]$. Using Eq. (A11) and

$$
-C_{\mathcal{O O T}} / B_{\mathcal{O}}=x_{\mathcal{O}} / \pi ; \quad \mathcal{O}=\epsilon, \varphi,
$$

$Q$ can be written as $\pi r_{12}^{2}\left\langle\epsilon\left(\mathbf{r}_{1}\right) \epsilon\left(\mathbf{r}_{2}\right) T_{\|,\|}\right\rangle_{\text {bulk }} / B_{\epsilon}$, and Eq. (3.56) is consistent with the small particle expansion if the stress tensor amplitude (3.53) is taken into account.

For $\langle\varphi \varphi\rangle_{\mathrm{DB}}$ all four even operators in Eqs. (3.49) and (3.53) contribute and lead to terms of order $L, L^{2}$, and $L^{3}$. For example, expanding the average (D1) for symmetric dumbbells leads to

$$
\begin{aligned}
\left\langle\varphi\left(\mathbf{r}_{1}\right) \varphi\left(\mathbf{r}_{2}\right)\right\rangle_{\mathrm{DB}}^{(i, i)} / B_{\varphi}= & r_{12}^{-1 / 4}\left\{1 \pm \pi L r_{12} /\left(8 r_{1} r_{2}\right)\right. \\
& -(\pi L)^{2} Q /(8 \times 24) \\
& \pm(\pi L)^{3}\left[r_{12} /\left(2^{8} r_{1} r_{2}\right)\right]\left[3 U_{+} / 2\right. \\
& \left.\left.+U_{12}-(I / 4)\right]+O\left(L^{4}\right)\right\},
\end{aligned}
$$

where $U_{+}, U_{12}, I$ are from Eq. (A8), and the upper and lower signs refer to the $(i, i)=(+,+)$ and (ord,ord) dumbbells of Figs. 1(b) and 1(a). Again one confirms that the $L^{2}$ term is consistent with the stress tensor contribution of the operator expansion. The terms of order $L$ and $L^{3}$ are consistent with the contributions from $\epsilon$ and $\Delta \epsilon, \partial_{\|}^{2} \epsilon$ as follows from Eq. (3.49), the value (3.46) of the bulk three-point amplitude $C_{\varphi \varphi \epsilon}$, the form $\mathcal{A}_{\epsilon}^{(i)} \pi^{3} / 4$ of $\mathcal{E}_{i i}^{\prime \prime}(0)$, and from identifying the curly bracket in Eq. (3.58) with that in Eq. (A5). The same operators and $L$-powers as above appear in the average $\langle\varphi \varphi\rangle_{\mathrm{DB}}^{(-,+)}$of Eq. (D4) for the $(-,+)$dumbbell of Fig. 1(c). However, consistency is now achieved by the different amplitudes $a_{1}^{(-,+)}, \ldots, b_{2}^{(-,+)}$from Eq. (3.51) and the last term in the square bracket of Eq. (3.53).

In the same way one confirms that the leading contributions to the mixed functions $\langle\varphi \epsilon\rangle_{\mathrm{DB}}^{(+,+)}$and $\langle\varphi \epsilon\rangle_{\mathrm{DB}}^{(-,+)}$in Eqs. (D3) and (D6) are of order $L^{1 / 8}, L^{2+(1 / 8)}$, and $L^{1+(1 / 8)}$, respectively, and are reproduced by the operators $\varphi,\left(\Delta, \partial_{\|}^{2}\right) \varphi$, and $\partial_{\|} \varphi$ with amplitudes given by Eqs. (3.50) and (3.52). Here one uses Eqs. (A7) and (A3).

We conclude with a remark on contributions with size exponent 4 . In the $d=2$ Ising model, the leading even operator, apart from the energy density $\epsilon$ and its derivatives, which plays the role of $\lambda$ in Eq. (2.3) is

$$
\lambda_{2}=T \bar{T},
$$

in terms of the usual ${ }^{13,23,25,34}$ complex components $T$ and $\bar{T}$ of the stress tensor $T_{\mu, \nu}$ in the $d=2$ dimensional $x-y$ plane. The operator $\lambda_{2}$ is isotropic and has scaling dimension $x_{\lambda_{2}}$ $=4$. Using well-known relations from conformal field theory in $d=2$ dimensions and the value $c=1 / 2$ for the conformal charge of the Ising model, one finds the amplitudes of the bulk two-point function and the normalized profile in the half plane

$$
B_{\lambda_{2}}=1 / 16, \quad \mathcal{A}_{\lambda_{2}}^{(+)}=\mathcal{A}_{\lambda_{2}}^{(\text {ord })}=1 / 16
$$

and the bulk three-point amplitudes

$$
C_{\mathcal{O O} \lambda_{2}} / B_{\mathcal{O}}=\left(x_{\mathcal{O}} / 2\right)^{2}
$$

for $\mathcal{O}=\epsilon$ or $\varphi$. The operator $\lambda_{2}$ gives the leading corrections to the bulk behavior in case of the energy two-point function in the half space ${ }^{23}$

$$
\left\langle\epsilon\left(\mathbf{r}_{1}\right) \epsilon\left(\mathbf{r}_{2}\right)\right\rangle_{\mathrm{hs}}^{(i)} / B_{\epsilon}-r_{12}^{-2}=r_{12}^{-2} Y\left(4 z_{1} z_{2} / r_{12}^{2}\right)
$$


and outside a circular particle with diameter $D=2 R$,

$$
\begin{aligned}
& \left\langle\boldsymbol{\epsilon}\left(\mathbf{r}_{1}\right) \boldsymbol{\epsilon}\left(\mathbf{r}_{2}\right)\right\rangle_{\mathrm{sph}}^{(i)} / B_{\epsilon}-r_{12}^{-2} \\
& \quad=r_{12}^{-2} Y\left[\left(\frac{r_{1} r_{2}}{R r_{12}}\right)^{2}\left(1-\frac{R^{2}}{r_{1}^{2}}\right)\left(1-\frac{R^{2}}{r_{2}^{2}}\right)\right] .
\end{aligned}
$$

Here the $z_{k}$ are the distances of $\mathbf{r}_{k}$ from the boundary of the half plane with $(i)=(+)$ or (ord) boundary conditions, $r_{k}$ are the distances from the particle center, and the scaling function $Y(\mathcal{Y})$ equals $\mathcal{Y}^{-1}-(\mathcal{Y}+1)^{-1}$ and tends to $\mathcal{Y}^{-2}$ for large $\mathcal{Y}$. In the product expansion (2.39) for the half space function $\langle\epsilon \epsilon\rangle_{\mathrm{hs}}$, the operators $\epsilon$, its derivatives, and $T_{\mu, \nu}$ do not contribute, since $\left\langle T_{\mu, \nu}\right\rangle_{\mathrm{hs}}=0$ and $C_{\epsilon \epsilon \epsilon}$ vanishes in the $d=2$ Ising model. The leading contribution to the right-hand side of Eq. (3.62) is $r_{12}^{2} /\left(16 z^{4}\right)$ with $z=\left(z_{1}+z_{2}\right) / 2$, which is consistent with the third term on the right-hand side of Eq. (2.39) with $\lambda$ replaced by $\lambda_{2}$ from Eq. (3.59). Correspondingly, in the small sphere expansion (2.20)-(2.24), $\epsilon$ and $\Delta \epsilon$ do not contribute to $\langle\epsilon \epsilon\rangle_{\mathrm{sph}}$, and it is the contribution from $a_{3}^{(i)} \lambda_{2}$ in Eq. (2.21) that dominates and is consistent with the behavior of Eq. (3.63) in the small particle limit. For the order parameter two-point function outside a circular particle the dominating contribution of order $D$ comes from the operator $\epsilon$, while $\Delta \epsilon$ and $\lambda_{2}$ lead to higher corrections of order $D^{3}$ and $D^{4}$. The $D^{4}$ contribution in the two cases is given by

$$
\begin{aligned}
& \left\langle\mathcal{O}\left(\mathbf{r}_{1}\right) \mathcal{O}\left(\mathbf{r}_{2}\right)\right\rangle_{\mathrm{sph}}^{(i)} /\left.B_{\mathcal{O}}\right|_{O\left(D^{4}\right)} \\
& \quad=\mathcal{A}_{\lambda_{2}}^{(i)} D^{4}\left\langle\mathcal{O}\left(\mathbf{r}_{1}\right) \mathcal{O}\left(\mathbf{r}_{2}\right) \lambda_{2}\right\rangle_{\text {bulk }} /\left(B_{\mathcal{O}} B_{\lambda_{2}}^{1 / 2}\right) \\
& \quad=x_{\mathcal{O}}^{2} r_{12}^{-2 x_{\mathcal{O}}}\left[R r_{12} /\left(r_{1} r_{2}\right)\right]^{4}
\end{aligned}
$$

with $\mathcal{O}=\epsilon$ or $\varphi$. For two-point functions $\langle\mathcal{O O}\rangle$ in strips and outside dumbbells we expect contributions of order $r_{12}^{4-2 x_{\mathcal{O}}}$ and $L^{4}$, respectively, not only from $\lambda_{2}$ but also from second derivatives of the stress tensor.

Generally the small particle expansion of an $N$-point function involves bulk averages of $N+1$ operators, as suggested by Eq. (2.30), with $\mathcal{O}(\mathbf{r})$ replaced by the product $\mathcal{O}_{1}\left(\mathbf{r}_{1}\right), \ldots \mathcal{O}_{N}\left(\mathbf{r}_{N}\right)$. As an example, for $N=3$ consider the order parameter three-point average (D11) in the presence of a $(+,+)$ dumbbell centered at the origin in $d=2$. Expanding Eq. (D11) for small dumbbell size $L$, one finds the leading contribution

$$
\begin{aligned}
& \left\langle\varphi\left(\mathbf{r}_{1}\right) \varphi\left(\mathbf{r}_{2}\right) \varphi\left(\mathbf{r}_{3}\right)\right\rangle_{\mathrm{DB}}^{(+,+)} / B_{\varphi}^{3 / 2} \\
& \quad \rightarrow(L / 2)^{1 / 8} \mathcal{F}_{++}(0)\left\langle\varphi\left(\mathbf{r}_{1}\right) \varphi\left(\mathbf{r}_{2}\right) \varphi\left(\mathbf{r}_{3}\right) \varphi(\mathbf{0})\right\rangle_{\text {bulk }} / B_{\varphi}^{2}
\end{aligned}
$$

with $\mathcal{F}_{++}(0)=(2 \pi)^{1 / 8}$ from Eqs. (3.44) and (3.45) and the bulk four-point function

$$
\begin{aligned}
& \left\langle\varphi\left(\mathbf{r}_{1}\right) \varphi\left(\mathbf{r}_{2}\right) \varphi\left(\mathbf{r}_{3}\right) \varphi(\mathbf{0})\right\rangle_{\text {bulk }} / B_{\varphi}^{2} \\
& \quad=2^{-1 / 2}\left(r_{12} r_{13} r_{23} r_{1} r_{2} r_{3}\right)^{-1 / 4}\left[r_{1} r_{23}+r_{2} r_{31}+r_{3} r_{12}\right]^{1 / 2} .
\end{aligned}
$$

This is consistent with the leading odd operator contribution $\tilde{a}_{1} \varphi$ in Eq. (2.18). Finally we note the corresponding expansion for a sphere in $d=2$ (i.e., a circular particle) with center at the origin and a diameter $D=2 R$. The three-point function $\langle\varphi \varphi \varphi\rangle_{\mathrm{sph}}^{(+)} / B_{\varphi}^{3 / 2}$, which is given by the right hand side of Eq.
(D11) with $(\Lambda \Lambda \Lambda / c c c)^{1 / 8}$ replaced by $\left\{D^{3} /\left[\left(r_{1}^{2}-R^{2}\right)\left(r_{2}^{2}\right.\right.\right.$ $\left.\left.\left.-R^{2}\right)\left(r_{3}^{2}-R^{2}\right)\right]\right\}^{1 / 8}$ and $u_{k l}$ by $\left\{1+\left[r_{k} r_{l} /\left(R r_{k l}\right)\right]^{2}[1\right.$ $\left.\left.-\left(R / r_{k}\right)^{2}\right]\left[1-\left(R / r_{l}\right)^{2}\right]\right\}^{1 / 4}$, tends, for small $R$, to the rhs of Eq. (3.65) with $(L / 2)^{1 / 8} \mathcal{F}_{++}(0)$ replaced by $\mathcal{A}_{\varphi}^{(+)} D^{1 / 8}$ $=(2 D)^{1 / 8}$. This is consistent with the small sphere leading operator contribution $\widetilde{a}_{1}^{(+)} \varphi$ in Eqs. (2.22) and (2.23).

\section{PARTICLE-WALL AND PARTICLE-PARTICLE INTERACTIONS}

Moving the colloidal dumbbell from infinity to a point $\mathbf{r}_{\mathrm{DB}}$ with a finite distance $z_{\mathrm{DB}}$ from the planar boundary wall of the critical fluid in the half space hs requires a free energy per $k_{B} T$ given by

$$
\begin{aligned}
\Psi & =-\ln \left[\left\langle e^{-\delta \mathcal{H}\left(\mathbf{r}_{\mathrm{DB}}\right)}\right\rangle_{\mathrm{hs}}^{(l)} /\left\langle e^{-\delta \mathcal{H}}\right\rangle_{\text {bulk }}\right] \\
& =-\ln \left\{\left[1+\left\langle\sigma\left(\mathbf{r}_{\mathrm{DB}}\right)\right\rangle_{\mathrm{hs}}^{(l)}\right] /\left(1+\langle\sigma\rangle_{\text {bulk }}\right)\right\} .
\end{aligned}
$$

Here $(l)=($ ord $),(+)$, or $(-)$ denotes the surface universality class of the boundary wall, and Eq. (2.1) has been used in the last step. Inserting $\sigma$ from Eqs. (2.2)-(2.8) yields, in case of a symmetry breaking wall $(l)=(+)$ or $(-)$, the leading isotropic and anisotropic contributions

$$
\Psi_{++}^{(l)} \rightarrow-\widetilde{a}_{1}\left\langle\varphi\left(\mathbf{r}_{\mathrm{DB}}\right)\right\rangle_{\mathrm{hs}}^{(l)}-\widetilde{b}_{1}(\cos \vartheta)^{2} \partial_{z_{\mathrm{DB}}}^{2}\left\langle\varphi\left(\mathbf{r}_{\mathrm{DB}}\right)\right\rangle_{\mathrm{hs}}^{(l)}
$$

to $\Psi$ for a $(+,+)$ dumbbell and

$$
\begin{aligned}
\Psi_{-+}^{(l)} \rightarrow & -a_{1}^{(-,+)}\left[\left\langle\epsilon\left(\mathbf{r}_{\mathrm{DB}}\right)\right\rangle_{\mathrm{hs}}^{(l)}-\langle\epsilon\rangle_{\mathrm{bulk}}\right] \\
& -\widetilde{c}_{1}(\cos \vartheta) \partial_{z_{\mathrm{DB}}}\left\langle\varphi\left(\mathbf{r}_{\mathrm{DB}}\right)\right\rangle_{\mathrm{hs}}^{(l)}
\end{aligned}
$$

for a $(-,+)$ dumbbell. Here $\vartheta$ is the angle between the surface normal of the wall and the dumbbell axis, which for the $(-,+)$ dumbbell points from the - to the + sphere. In Eqs. (4.2) and (4.3) we have assumed $\langle\varphi\rangle_{\text {bulk }}=0$, which applies at the critical composition, and $T \geqslant T_{c}$. At the critical point $T=T_{c}$ the amplitudes $a, b, c$ given in Eqs. (2.18) and (2.19) and the half space profiles given below Eq. (2.13) lead to

$$
\begin{aligned}
\Psi_{++}^{(l)} \rightarrow & -\left(\frac{L}{2 z_{\mathrm{DB}}}\right)^{x_{\varphi}} \mathcal{A}_{\varphi}^{(l)}\left[\mathcal{F}_{++}(0)\right. \\
& \left.+(\cos \vartheta)^{2}\left(\frac{L}{2 z_{\mathrm{DB}}}\right)^{2} \mathcal{F}_{++}^{\prime \prime}(0) / 2\right]
\end{aligned}
$$

and

$$
\begin{aligned}
\Psi_{-+}^{(l)} & \rightarrow\left(\frac{L}{2 z_{\mathrm{DB}}}\right)^{x_{\epsilon}}\left(-\mathcal{A}_{\epsilon}^{(l)}\right) \mathcal{E}_{-+}(0)+(\cos \vartheta) \\
& \times\left(\frac{L}{2 z_{\mathrm{DB}}}\right)^{x_{\varphi}+1} \mathcal{A}_{\varphi}^{(l)} \mathcal{F}_{-+}^{\prime}(0) .
\end{aligned}
$$

In the cases (i), (ii), (iii) shown in Fig. 2 the leading terms of the interaction between the dumbbell and the wall are isotropic and attractive, repulsive, repulsive, respectively, since $\mathcal{A}_{\varphi}^{(+)}=-\mathcal{A}_{\varphi}^{(-)},-\mathcal{A}_{\epsilon}^{(+)}, \mathcal{F}_{++}(0), \mathcal{E}_{-+}(0)$ in Eqs. (4.4) and (4.5) are positive. Since $\mathcal{F}_{++}^{\prime \prime}(0)$ and $\mathcal{F}_{-+}^{\prime}(0)$ are also positive, the leading anisotropic parts of the interactions favor the dumbbell orientations shown in Fig. 2. 


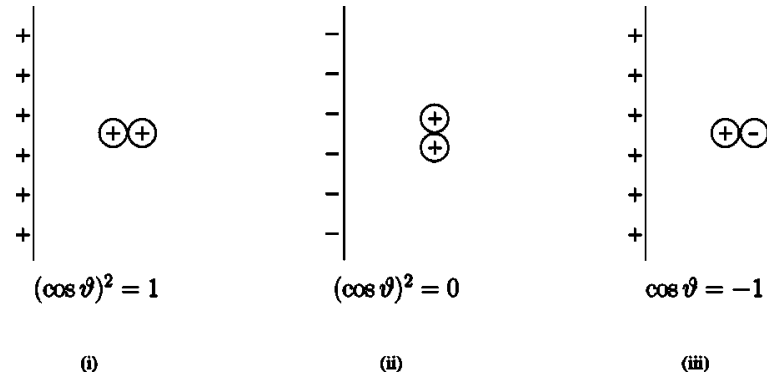

FIG. 2. Dumbbell orientations favored by a wall.

The inversion (2.25) about the center of the dumbbell, which maps its surface onto the two parallel boundaries of a film with width $2 L$, maps the planar boundary wall of the half space onto the surface of a spherical particle, which is located inside the film, has a surface universality class $l$, and a diameter $D$ related by $D=L^{2} / z_{\mathrm{DB}}$ to the dumbbell-wall distance $z_{\mathrm{DB}}$. At the critical point of the solution the free energy $\Psi$ introduced above equals the free energy

$$
\psi_{l}=-\ln \left(1+\left\langle\sigma_{\mathrm{sph}}^{(l)}\right\rangle_{\text {film }}\right)
$$

required to move the sphere from bulk solution to its position inside the film. Here $\sigma_{\text {sph }}$ denotes the sum of weighted operators in the small sphere expansion described in Eqs. (2.20)-(2.24). In the favorable configuration of Fig. 2(i), the $\hat{z}$ direction normal to the film boundaries is horizontal, and the sphere center is located at $\hat{z}=-D / 2$, a distance $D / 2$ away from the center of the film. In the corresponding unfavorable configuration [not shown in Fig. 2(i)] with the dumbbell parallel to the wall, the film normal is vertical, and the sphere is located in the film center at $\hat{z}=0$. Thus for $l=+$,

$$
\begin{aligned}
\left.\psi_{+}\right|_{\{\text {favor;unfav }\}} \rightarrow & -\mathcal{A}_{\varphi}^{(+)} D^{x_{\varphi}}(2 L)^{-x_{\varphi}} \\
& \times\left\{\mathcal{F}_{++}[-D /(2 L)] ; \mathcal{F}_{++}(0)\right\},
\end{aligned}
$$

apart from nonleading contributions. Expanding the even function $\mathcal{F}_{++}$for small $D=L^{2} / z_{\mathrm{DB}}$ leads to a result consistent with $\Psi_{++}^{(+)}$in Eq. (4.4), since $(\cos \vartheta)^{2}=\{1 ; 0\}$ for the \{favorable; unfavorable $\}$ orientations with $\vartheta=\{0 ; \pi / 2\}$. The next to leading isotropic contribution in $\Psi$ from the dumbbell operator $\Delta \varphi$ in Eq. (2.18) is reproduced by the contribution to $\psi$ from the next to leading sphere operator $S_{\Delta \varphi}^{(+)}$in Eq. (2.23).

In the favorable configuration of the $(-,+)$ dumbbell with orientation $\vartheta=\pi$ shown in Fig. 2(iii), the mapping leads to a $(+)$ sphere at $\hat{z}=-D / 2$ inside a $(+,-)$ film with horizontal film normal, while in the unfavorable configuration with $\vartheta=0$ (not shown) the sphere is located at the same position inside a $(-,+)$ film. Thus

$$
\begin{aligned}
\left.\psi_{+}\right|_{\{\text {favor,unfav }\}} \rightarrow & -\mathcal{A}_{\epsilon}^{(+)} D^{x} \varepsilon(2 L)^{-x_{\epsilon}} \mathcal{E}_{\{+-;-+\}}(-D /(2 L)) \\
& -\mathcal{A}_{\varphi}^{(+)} D^{x} \varphi(2 L)^{-x_{\varphi}} \mathcal{F}_{\{+-;-+\}}(-D /(2 L))
\end{aligned}
$$

for the ffavorable; unfavorable $\}$ orientations with $\cos \vartheta$ $=\{-1 ; 1\}$. In leading isotropic and anisotropic orders the two even functions $\mathcal{E}_{+-}(-D /(2 L)), \mathcal{E}_{-+}(-D /(2 L))$ can be replaced by $\mathcal{E}_{-+}(0)$, the two odd functions $\mathcal{F}_{+-}(-D /(2 L))$,
$\mathcal{F}_{-+}(-D /(2 L))$ by $-(\cos \vartheta)[D /(2 L)] \mathcal{F}_{-+}^{\prime}(0)$, and $\psi_{+}$in Eq. (4.8) is consistent with $\Psi_{-+}^{(+)}$in Eq. (4.5).

The small particle operator expansion (2.1)-(2.8) with the dumbbell weights (2.9)-(2.19) applies in the vicinity of the critical point whenever the correlation length $\xi$ is much larger than the particle size, see the remark at the end of Sec. II A. The leading isotropic and anisotropic particle-wall interactions at the critical composition (vanishing orderparameter average in the bulk) above $T_{c}$ are given by Eqs. (4.2) and (4.3), with the half space profiles $\langle\varphi\rangle_{\mathrm{hs}}$ and $\langle\epsilon\rangle_{\mathrm{hs}}$ for $T$ above $T_{c}$. The profiles depend on the ratio $z_{\mathrm{DB}} / \xi$. The expressions (4.2) and (4.3) for the interactions apply for $L$ $\ll z_{\mathrm{DB}}, \quad \xi$ in the entire region extending from $z_{\mathrm{DB}} / \xi \ll 1$, where they reduce to the critical point expressions (4.4) and (4.5) with power law decay in $z_{\mathrm{DB}}$, to $z_{\mathrm{DB}} / \xi \gg 1$ where they decay exponentially. The temperature dependence ${ }^{9,10}$ of $\partial_{z_{\mathrm{DB}}}\langle\varphi\rangle_{\mathrm{hs}}$ and $\partial_{z_{\mathrm{DB}}}^{2}\langle\varphi\rangle_{\mathrm{hs}}$ implies that the reduced free energy cost $\Delta \Psi$ to turn the $(+,+)$ or $(-,+)$ dumbbell at fixed $z_{\mathrm{DB}}$ from the favorable to the unfavorable orientation has a maximum not at but above $T_{c}$.

It is interesting to compare the solvation free energy $\Delta F_{++}^{(+)}=k_{B} T_{c}\left[L /\left(2 z_{\mathrm{DB}}\right)\right]^{x_{\varphi}+2} \mathcal{A}_{\varphi}^{(+)} \mathcal{F}_{++}^{\prime \prime}(0) / 2$ at the critical point it costs to turn the $(+,+)$ dumbbell near a $(+)$ wall from the favorable $\vartheta=0$ to the unfavorable $\vartheta=\pi / 2$ orientation, see Eq. (4.4) and Fig 2(i), with the corresponding energy change $\Delta E$ due to the van der Waals attraction. In the nonretarded Hamaker approximation one finds $\Delta E=A(8 / 3)$ $\times\left[L /\left(2 z_{\mathrm{DB}}\right)\right]^{5}$ for the small dumbbell in $d=3$, with $A$ the Hamaker constant. This follows from differentiating twice the corresponding interaction between a wall and a sphere ${ }^{35}$ with respect to the wall-sphere distance. Since $x_{\varphi}+2=2.51$ in $d=3$ is smaller than the exponent 5 , and since $L / z_{\mathrm{DB}}$ is small, the solvation free energy dominates. For a rough quantitative estimate we use the values $\mathcal{F}_{++}^{\prime \prime}(0) / \mathcal{A}_{\varphi}^{(+)} \approx 2.5$ and $\mathcal{A}_{\varphi}^{(+)} \approx 1.9$ in $d=3$. The first follows from Monte Carlo simulations $^{36}$ of a $d=3$ Ising model between parallel plates and has a magnitude in between the values $K^{3} \sqrt{2}=9.01$ and $\left(\pi^{17 / 8}\right) / 32=0.36$ in $d=4$ and $d=2$ dimensions, see Eqs. (3.3) and (3.44). The second was estimated ${ }^{37}$ from an interpolation formula $6 \varepsilon^{-1 / 2}(1-\delta \varepsilon) /\{1+[(31 / 27)-\delta] \varepsilon\}$ for $2^{x} \mathcal{A}_{\varphi}^{(+)}$, which is consistent with the $\varepsilon$-expansion ${ }^{7}$ and reproduces the value $2^{x} \mathcal{A}_{\varphi}^{(+)}=2^{1 / 4}$ in $d=2$ if one chooses $\delta$ $=0.053$. For a water-lutidine mixture ${ }^{10,11}$ with $T_{c}=307 \mathrm{~K}$ and a Hamaker constant $A \approx 10^{-20} J$, the rough estimate then is $\Delta F_{++}^{(+)} /\left(10^{-20} J\right) \approx 1.9\left[L /\left(2 z_{\mathrm{DB}}\right)\right]^{2.51}$ and $\Delta E /\left(10^{-20} J\right)$ $\approx 2.7\left[L /\left(2 z_{\mathrm{DB}}\right)\right]^{5}$. We also note the $d=3$ estimate $\mathcal{A}_{\varphi}^{(+)} \mathcal{F}_{++}(0) \approx 6.2$ of the universal amplitude which appears in the isotropic leading interaction between a $(+,+)$ dumbbell and a $(+)$ wall in Eq. (4.4). The $d=3$ Monte Carlo $^{36}$ result $\mathcal{F}_{++}(0) / \mathcal{A}_{\varphi}^{(+)}=1.74$ is not far from the result 1.88 of a linear interpolation between the values $\sqrt{2} K=2.62$ and $\pi^{1 / 8}=1.15$ in $d=4$ and $d=2$ from Eqs. (3.1), (3.3), and (3.44).

Finally consider two dumbbells $P, Q$ in unbounded space. The free energy per $k_{B} T$ required to move them from infinite separation to center positions $\mathbf{r}_{P}$ and $\mathbf{r}_{Q}$, with their axes oriented along the unit vectors $\varrho_{P}$ and $\varrho_{Q}$, is given by 


$$
\begin{aligned}
\Upsilon_{P, Q} & =-\ln \frac{\left\langle e^{-\delta \mathcal{H}_{P}\left(\mathbf{r}_{P}\right)} e^{-\delta \mathcal{H}_{Q}\left(\mathbf{r}_{Q}\right)}\right\rangle_{\text {bulk }}}{\left\langle e^{\left.-\delta \mathcal{H}_{P}\right\rangle_{\text {bulk }}\left\langle e^{-\delta \mathcal{H}_{Q}}\right\rangle_{\text {bulk }}}\right.} \\
& =-\ln \frac{\left\langle\left[1+\sigma_{P}\left(\mathbf{r}_{P}\right)\right]\left[1+\sigma_{Q}\left(\mathbf{r}_{Q}\right)\right]\right\rangle_{\text {bulk }}}{\left\langle 1+\sigma_{P}\right\rangle_{\text {bulk }}\left\langle 1+\sigma_{Q}\right\rangle_{\text {bulk }}},
\end{aligned}
$$

which equals $-\left\langle\sigma_{P}\left(\mathbf{r}_{P}\right) \sigma_{Q}\left(\mathbf{r}_{Q}\right)\right\rangle_{\text {bulk }}+\left\langle\sigma_{P}\right\rangle_{\text {bulk }}\left\langle\sigma_{Q}\right\rangle_{\text {bulk }}$ to second order in $\sigma$. Here $\sigma_{P}$ and $\sigma_{Q}$ are the operator contributions from dumbbells $P$ and $Q$. For simplicity now consider two dumbbells of equal size $L$ in a solvent right at the critical point. For two $(-,+)$ dumbbells the leading isotropic and anisotropic interactions come from the bulk averages of $\epsilon \epsilon$ and $\left(\partial_{\| P} \varphi\right)\left(\partial_{\| Q} \varphi\right)$, with the result

$$
\begin{aligned}
\Upsilon_{P, Q} \rightarrow & -\left(\frac{L}{2 r_{P Q}}\right)^{2 x_{\epsilon}}\left[\mathcal{E}_{-+}(0)\right]^{2} \\
& -\left(\frac{L}{2 r_{P Q}}\right)^{2 x_{\varphi}+2}\left[\mathcal{F}_{-+}^{\prime}(0)\right]^{2}\left(2 / x_{\varphi}\right)\left[\left(\varrho_{P} \varrho_{Q}\right)\right. \\
& \left.-\left(2 x_{\varphi}+2\right) \frac{\left(\varrho_{P} \mathbf{r}_{P Q}\right)\left(\varrho_{Q} \mathbf{r}_{P Q}\right)}{r_{P Q}^{2}}\right] .
\end{aligned}
$$

In $d=3$ spatial dimensions the anisotropic part of the interaction is very close to the classical dipole-dipole form, since $2 x_{\varphi}+2=d+\eta$ and the exponent $\eta$ is very small. However, the overall sign is different, since, contrary to electric dipoles, for the "dipolar" colloidal dumbbell of Fig. 1(c) it is energetically favorable for equal polarities to be close to each other. Note that the actual "dipole strength" $\widetilde{c}_{1} \partial_{\|} \varphi$ of the $(-,+)$ dumbbell in Eq. (2.19) is larger by a factor $2^{-\left(x_{\varphi}+1\right)} x_{\varphi}^{-1} \mathcal{F}_{-+}^{\prime}(0) / \mathcal{A}_{\varphi}^{(+)}$than the superposition $\widetilde{a}_{1}^{(+)}[\varphi(L / 2)-\varphi(-L / 2)] \rightarrow \widetilde{a}_{1}^{(+)} L \partial_{\|}^{2} \varphi$ of sphere strengths, with $\widetilde{a}_{1}^{(+)}$the weight for a single $(+)$sphere of diameter $D$ $=L$ from Eqs. (2.22) and (2.23). The above factor equals $K^{2} / 2=1.72$ and $4(\pi / 2)^{9 / 8}=6.65$ in $d=4$ and $d=2$ dimensions and can be traced back to the ratio of the first derivatives at $y=0$ of the actual profile $\mathcal{F}_{-+}(y)$ between parallel $(-,+)$ plates and its superposition approximation $2^{x_{\varphi}} \mathcal{A}_{\varphi}^{(+)}\left[-(1+y)^{-x_{\varphi}}+(1-y)^{-x_{\varphi}}\right]$ following from Eq. (2.13). For two $(+,+)$ dumbbells the bulk averages of $\varphi \varphi$ and $\left(\partial_{\| P}\right) \varphi+\varphi\left(\partial_{\| Q}\right)$ yield the leading isotropic and anisotropic interactions

$$
\begin{aligned}
\Upsilon_{P, Q} \rightarrow & -\left(\frac{L}{2 r_{P Q}}\right)^{2 x_{\varphi}}\left[\mathcal{F}_{++}(0)\right]^{2} \\
& -\left(\frac{L}{2 r_{P Q}}\right)^{2 x_{\varphi}+2} 2 \mathcal{F}_{++}(0) \mathcal{F}_{++}^{\prime \prime}(0)\left[\left(\frac{\left(\varrho_{P} \mathbf{r}_{P Q}\right)}{r_{P Q}}\right)^{2}\right. \\
& \left.+\left(\frac{\left(\varrho_{Q} \mathbf{r}_{P Q}\right)}{r_{P Q}}\right)^{2}\right],
\end{aligned}
$$

showing that an orientation with both dumbbell axes parallel to the center-to-center distance vector $\mathbf{r}_{P Q}$ is energetically most favorable.

\section{DUMBBELL OF TWO OVERLAPPING SPHERES AND LENS}

Besides the dumbbells composed of two touching spheres in Fig. 1, dumbbells of two overlapping spheres and
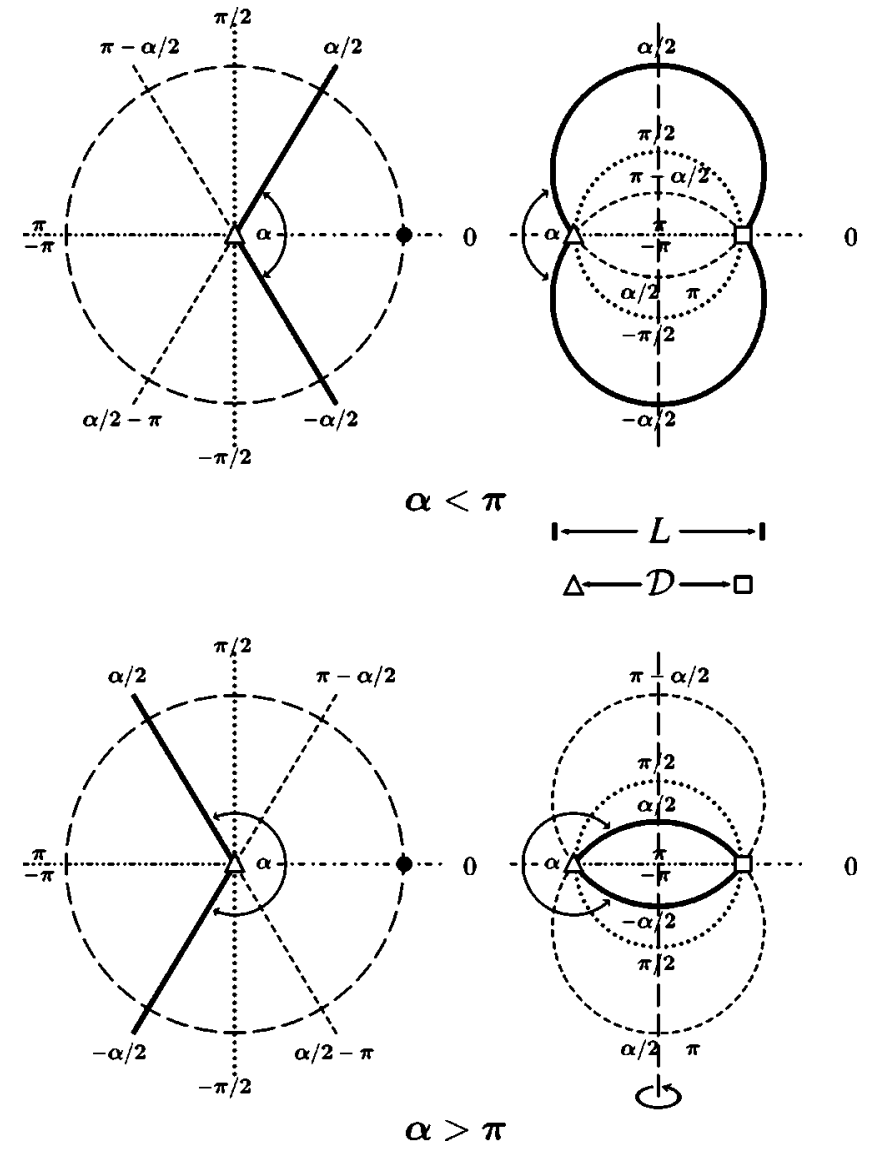

FIG. 3. Conformal mapping of a dumbbell or lens onto a wedge.

particles in the shape of a lens with two spherical surfaces are of interest ${ }^{22}$ and can be considered just as easily. The reason is that a critical system containing one such particle can be conformally mapped onto a critical system filling a wedge with opening angle $\alpha$, which is smaller or larger than $\pi$ in case of the dumbbell or lens, as in Fig. 3. For $\alpha=0, \pi$, and $2 \pi$ the particle in Fig. 3 degenerates to the touchingsphere dumbbell of Fig. 1, a spherical particle, and a circular disk, respectively.

\section{A. Density profiles in a wedge}

For a wedge the density profiles of scalar operators have the form

$$
\left\langle\mathcal{O}\left(\hat{\mathbf{r}}_{\|}, \rho, \Omega\right)\right\rangle_{\text {wedge }}^{(i, j)}=B_{\mathcal{O}}^{1 / 2} \rho^{-x_{\mathcal{O}}} \overline{\mathcal{P}}_{i, j}(\alpha, \Omega),
$$

and for $\mathcal{O}=\epsilon, \lambda, \varphi$ we denote $\overline{\mathcal{P}}$ by $\overline{\mathcal{E}}, \overline{\mathcal{L}}, \overline{\mathcal{F}}$. Here the position vector $\hat{\mathbf{r}}$ is expressed in cylindrical polar coordinates $\left(\hat{\mathbf{r}}_{\|}, \rho, \Omega\right)$, where the edge of the wedge is the axis. The component $\hat{\mathbf{r}}_{\|}$is parallel to the edge (and, in general, has dimension $d-2$ ), and the two component vector perpendicular to the edge is determined by its angle $\Omega$ with the symmetry half plane of the wedge, i.e., $-\alpha / 2 \leqslant \Omega \leqslant \alpha / 2$, and by the distance $\rho$ from the edge. The indices $(i, j)$ characterize the surface universality classes of the two boundary half planes $\Omega=(-\alpha / 2, \alpha / 2)$ of the wedge.

For $\alpha=\pi$ the wedge equals the half space, and for equal boundary conditions $i=j$, 


$$
\overline{\mathcal{P}}_{i, i}(\pi, \Omega)=\mathcal{A}_{\mathcal{O}}^{(i)}(\cos \Omega)^{-x_{\mathcal{O}}} .
$$

For $\alpha \rightarrow 0$ the wedge tends to the film geometry of Eq. (2.11), where $2 \Omega / \alpha \rightarrow \hat{z} / L, \rho \Omega \rightarrow \hat{z}$, and

$$
\overline{\mathcal{P}}_{i, j}(\alpha, \Omega) \rightarrow \alpha^{-x_{\mathcal{O}}} \mathcal{P}_{i, j}(2 \Omega / \alpha), \alpha \rightarrow 0,
$$

with the film scaling function $\mathcal{P}_{i, j}$ from Eq. (2.11). For $\Omega$ $\rightarrow[-\alpha / 2, \alpha / 2]$ the boundary half planes of the wedge are approached, and

$$
\overline{\mathcal{P}}_{i, j}(\alpha, \Omega) \rightarrow\left[\mathcal{A}_{\mathcal{O}}^{(i)}(\Omega+\alpha / 2)^{-x_{\mathcal{O}}, \mathcal{A}_{\mathcal{O}}^{(j)}}(-\Omega+\alpha / 2)^{-x_{\mathcal{O}}}\right] .
$$

Equations (5.3) and (5.4) are consistent with Eq. (2.13). The small particle amplitudes follow from the behavior of $\overline{\mathcal{P}}$ for $|\Omega| \ll \alpha / 2$. For equal boundary conditions $i=j$ the function $\mathcal{P}$ is even in $\Omega$, and

$$
\overline{\mathcal{P}}_{i, i}(\alpha, \Omega)=p_{0}^{(i)}(\alpha)\left[1+p_{2}^{(i)}(\alpha) \Omega^{2} / 2+\cdots\right] .
$$

Explicit expressions are available for $d=2$ and $d / 4$. For the order parameter profile $\langle\varphi\rangle_{\text {wedge }}^{(+,+)}$in a wedge with $(+$, $+)$ boundary conditions, one finds ${ }^{6,38}$ for the scaling functions $\overline{\mathcal{P}}_{i, i}=\overline{\mathcal{F}}_{++}$, where

$$
\overline{\mathcal{F}}_{++} / \mathcal{A}_{\varphi}^{(+)}=\left(\frac{\alpha}{\pi} \cos \frac{\pi \Omega}{\alpha}\right)^{-1 / 8}
$$

for $d=2$, and

$$
\begin{aligned}
\overline{\mathcal{F}}_{++} / \mathcal{A}_{\varphi}^{(+)}= & \left\{\left(\frac{1-m}{1-2 m}\right)^{1 / 2} \frac{1}{\operatorname{cn}(\Omega / \sqrt{1-2 m} ; m)} ;\right. \\
& \left.\left(\frac{1}{1+m}\right)^{1 / 2} \frac{\operatorname{dn}(\Omega / \sqrt{1+m} ; m)}{\operatorname{cn}(\Omega / \sqrt{1+m} ; m)}\right\}
\end{aligned}
$$

for $d=4$ with $\{\alpha \leqslant \pi ; \pi \leqslant \alpha\}$. Here cn and dn are Jacobian elliptic functions and $m=m(\alpha)$ is determined by $\alpha$ $=2 K(m)\{\sqrt{1-2 m} ; \sqrt{1+m}\}$ in the two cases, with $K(m)$ the complete elliptic integral of the first kind. Thus the two branches $1 / 2 \geqslant m \geqslant 0$ and $0 \leqslant m \leqslant m_{2 \pi} \equiv 0.827$ for $0 \leqslant \alpha \leqslant \pi$ and $\pi \leqslant \alpha \leqslant 2 \pi$ meet at $\alpha=\pi$ where $m=0$.

The small $\Omega$ amplitudes in Eq. (5.5) are given by

$$
\begin{aligned}
p_{0}^{(+)}(\alpha) & =f_{0}^{(+)}(\alpha) \\
& \equiv \mathcal{A}_{\varphi}^{(+)}\left[\left(\frac{\pi}{\alpha}\right)^{1 / 8} ;\left\{\left(\frac{1-m}{1-2 m}\right)^{1 / 2} ;\left(\frac{1}{1+m}\right)^{1 / 2}\right\}\right]
\end{aligned}
$$

and

$$
p_{2}^{(+)}(\alpha)=f_{2}^{(+)}(\alpha) \equiv\left[\frac{1}{8}\left(\frac{\pi}{\alpha}\right)^{2} ;\left\{\frac{1}{1-2 m} ; \frac{1-m}{1+m}\right\}\right]
$$

for $[d=2 ;\{d=4, \alpha \leqslant \pi ; d=4, \pi \leqslant \alpha\}]$.

\section{B. Overlapping spheres and lens}

An inversion about the point denoted by the heavy dot on the left hand sides of Fig. 3 maps the interior of the wedge onto the exterior of a particle with a dumbbell or lens shape. This point is located in the half plane $\Omega=0$, and we choose it in the two-dimensional plane $\hat{\mathbf{r}}_{\|}=0$ perpendicular to the edge. The two boundary half planes of the wedge and the edge where they meet are mapped onto the two spherical boundary surfaces of the particle and the circle $C$ of diameter $\mathcal{D}$ where they intersect. The points $\hat{\mathbf{r}}=0$ (triangle) and $\hat{\mathbf{r}}$ $=\infty$ of the wedge geometry are mapped onto the two points on $C$ denoted by a triangle and a square, respectively. $C$ is located in the symmetry plane of the particle, and its exterior and interior are the images of the interior and exterior symmetry half planes $\Omega=0$ and $\Omega= \pm \pi$ of the wedge. These are denoted by dot-dash and dot-dot-dash lines, respectively, in Fig. 3. The symmetry axis of the particle is the image of a circle in the plane $\hat{\mathbf{r}}_{\|}=0$ of the wedge with center in the edge and passing through the center of inversion, see the long dashes in Fig. 3.

A point $\mathbf{r}$ in the particle geometry with distance $r$ from the particle center and component $r_{\|}$parallel to the particle rotation axis originates from a point $\hat{\mathbf{r}}$ in the wedge geometry with

$$
\operatorname{tg} \Omega=\frac{\mathcal{D} r_{\|}}{r^{2}-(\mathcal{D} / 2)^{2}}, \quad \operatorname{sgn} \Omega=\operatorname{sgn} r_{\|}, \quad-\pi \leqslant \Omega \leqslant \pi .
$$

The two hemispheres $r=\mathcal{D} / 2$ with $r_{\|}<0$ and $>0$ in the particle geometry are the images of the two half planes with $\Omega=-\pi / 2$ and $\pi / 2$ in the wedge geometry, see the dotted lines in Fig. 3. Equation (5.10) determines the images of the half planes with arbitrary $\Omega$. These are portions of spherical surfaces, all bounded by the image circle $C$ of the edge.

The upper boundary surface of the particle with $\Omega$ $=\alpha / 2$ is part of a sphere with radius $(\mathcal{D} / 2) / \sin (\alpha / 2)$ and a center which has an $r_{\|}$component of $(\mathcal{D} / 2) \operatorname{ctg}(\alpha / 2)$ and is located above or below the symmetry plane $r_{\|}=0$ in the dumbbell or lens case, with $\alpha<\pi$ or $\alpha>\pi$. Thus the two overlapping spherical surfaces of diameter

$$
L=\mathcal{D} / \sin (\alpha / 2)
$$

of the dumbbell have a center-to-center distance

$$
d_{\mathrm{cc}}=L \cos (\alpha / 2),
$$

which tends to $L$ and 0 , respectively, for $\alpha \rightarrow 0$ (two touching spheres) and $\alpha \rightarrow \pi$ (single sphere). The lens of diameter $\mathcal{D}$ has a width

$$
\mathcal{W}=\mathcal{D} \operatorname{ctg}(\alpha / 4)=\mathcal{D} \operatorname{tg}\left(\alpha^{\prime} / 4\right)
$$

with $\alpha^{\prime}=2 \pi-\alpha$ the internal lens angle. The width $\mathcal{W}$ tends to $\mathcal{D}$ for $\alpha \rightarrow \pi$ (single sphere) and to $\mathcal{D} \alpha^{\prime} / 4$ near the disk limit $\alpha^{\prime} \rightarrow 0$.

Adapting the transformation laws (2.26), (2.27) to this mapping and using Eq. (5.1) leads to the result

$$
\langle\mathcal{O}(\mathbf{r})\rangle_{\text {particle }}^{(i, j)}=B_{\mathcal{O}}^{1 / 2}\left\{\frac{\mathcal{D}^{2}}{\left[r^{2}-(\mathcal{D} / 2)^{2}\right]^{2}+\mathcal{D}^{2} r_{\|}^{2}}\right\}^{x_{\mathcal{O}} / 2} \overline{\mathcal{P}}_{i, j}(\alpha, \Omega)
$$

for the profile of a scalar operator $\mathcal{O}$ outside a dumbbell or lens. Here $\Omega$ depends on $\mathbf{r}$ as given by Eq. (5.10). The expression in curly brackets in Eq. (5.14) equals $[b(\hat{\mathbf{r}}) / \rho(\hat{\mathbf{r}})]^{2}$, with $b$ the dilatation factor, and is invariant under rotation about the particle axis, as required by symmetry. 
Note that Eq. (5.14) reduces to the profile (2.28) outside two touching spheres as $\alpha \rightarrow 0$ for fixed sphere diameter $L$ in Eq. (5.11). Here one uses the limiting behavior (5.3) of $\overline{\mathcal{P}}$ and the relation $\Omega \rightarrow \alpha L r_{\|} /\left(2 r^{2}\right)$, which follows from Eq. (5.10). In the limit $\alpha \rightarrow \pi$, Eq. (5.14) for $i=j$ reduces to the isotropic profile ${ }^{6}$

$$
\langle\mathcal{O}(\mathbf{r})\rangle_{\text {sphere }}^{(i)}=B_{\mathcal{O}}^{1 / 2} \mathcal{A}_{\mathcal{O}}^{(i)}\left(\frac{\mathcal{D}}{r^{2}-(\mathcal{D} / 2)^{2}}\right)^{x_{\mathcal{O}}}
$$

outside a single sphere with diameter $\mathcal{D}$. Here one uses the behavior (5.2) of $\overline{\mathcal{P}}$, and the anisotropy drops out on expressing $\Omega$ in terms of $\mathbf{r}$ via Eq. (5.10).

One may check that the leading behavior of the profile (5.14) close to the particle surface equals the behavior near the planar boundary surface of a half space: On approaching the upper particle boundary $\Omega=\alpha / 2$ in Fig. 3 from outside along a line which passes through the center of the belonging sphere and forms an angle $\gamma$ with the particle axis, $\Omega$ from Eq. (5.10) approaches $\alpha / 2$ from below according to

$$
\frac{\alpha}{2}-\Omega \rightarrow \frac{2 \delta}{\mathcal{D}} \frac{[\sin (\alpha / 2)]^{2}}{\cos (\alpha / 2)+\cos \gamma}, \quad 0 \leqslant \gamma<\pi-\frac{\alpha}{2}
$$

as the distance $\delta$ from the boundary tends to zero. Since the quantity in curly brackets in Eq. (5.14) approaches $[\sin (\alpha / 2)]^{2} /\{[\cos (\alpha / 2)+\cos \gamma] \mathcal{D} / 2\}^{2}$, the angles $\alpha$ and $\gamma$ drop out on substituting Eq. (5.4) with Eq. (5.16) into Eq. (5.14), and one finds the expected half space behavior $B_{\mathcal{O}}^{1 / 2} \mathcal{A}_{\mathcal{O}}^{(j)} \delta^{-x_{\mathcal{O}}}$ for $\langle\mathcal{O}\rangle_{\text {particle }}^{(i, j)}$.

Amplitudes of the small particle expansion follow from expanding Eq. (5.14) for $\mathcal{D} \ll r$. For equal boundary conditions $(i, j)=(+,+)$ or (ord,ord), Eqs. (5.14), (5.10), and (5.5) lead to

$$
\begin{aligned}
\langle\mathcal{O}(\mathbf{r})\rangle_{\text {particle }}^{(i, i)}= & B_{\mathcal{O}}^{1 / 2} p_{0}^{(i)}(\alpha)\left(\frac{\mathcal{D}}{r^{2}}\right)^{x_{\mathcal{O}}}\left\{1+\frac{\mathcal{D}^{2}}{2 r^{2}}\left[\frac{x_{\mathcal{O}}}{2}\right.\right. \\
& \left.\left.+\left[p_{2}^{(i)}(\alpha)-x_{\mathcal{O}}\right] \frac{r_{\|}^{2}}{r^{2}}\right]\right\}
\end{aligned}
$$

up to order $\mathcal{D}^{x_{\mathcal{O}^{+2}}}$. For a $(i, i)=(+,+)$ particle, Eq. (5.17) with $\mathcal{O}=\varphi$ determines the amplitudes $\widetilde{a}_{1}, \tilde{b}_{1}$ of the leading isotropic and anisotropic operators $\varphi$ and $\partial_{\|}^{2} \varphi$ in the small particle expansion (2.1)-(2.6). Proceeding as in the derivation of Eq. (2.18) from Eqs. (2.32) and (2.34), one finds

$$
\widetilde{a}_{1}=\mathcal{D}^{x_{\varphi}} f_{0}^{(+)}(\alpha) / B_{\varphi}^{1 / 2}
$$

and

$$
\begin{aligned}
\tilde{b}_{1} / \widetilde{a}_{1} & =\frac{\mathcal{D}^{2}}{8 x_{\varphi}\left(x_{\varphi}+1\right)}\left[f_{2}^{(+)}(\alpha)-x_{\varphi}\right] \\
& =\mathcal{D}^{2}\left[\frac{1}{9}\left(\left(\frac{\pi}{\alpha}\right)^{2}-1\right) ;\left\{\frac{m / 8}{1-2 m} ; \frac{-m / 8}{1+m}\right\}\right] .
\end{aligned}
$$

Here $f_{0,2}^{(+)}$are the small $\Omega$ amplitudes in Eqs. (5.5), (5.8), and (5.9), and the last line in Eq. (5.19) applies to $[d=2 ;\{d$ $=4, \alpha \leqslant \pi ; d=4, \pi \leqslant \alpha\}]$. The results (5.18) and (5.19) should be compared with Eq. (2.18) for two touching spheres. As suggested by Fig. 3, the former reduce to the latter as the angle $\alpha$ in Eqs. (5.18), (5.19), and (5.11) tends to zero. This can be explicitly shown by using that, due to Eq. (5.3), the coefficients $p_{0}^{(i)}$ and $p_{0}^{(i)} p_{2}^{(i)}$ in Eq. (5.5) tend to $\alpha^{-x_{\mathcal{O}} \mathcal{P}_{i, i}(0)}$ and $4 \alpha^{-\left(x_{\mathcal{O}^{+2}}\right)} \mathcal{P}_{i, i}^{\prime \prime}(0)$, respectively, as $\alpha$ tends to zero. For $\alpha \rightarrow \pi$ the coefficient $p_{2}^{(i)}$ tends to $x_{\mathcal{O}}$ [see Eq. (5.2)], and Eqs. (5.18) and (5.19) reduce to the single-sphere results $\widetilde{a}_{1}=\mathcal{D}^{x} \varphi \mathcal{A}_{\mathcal{O}}^{(+)} / B_{\varphi}^{1 / 2}$ and $\widetilde{b}_{1}=0$.

Equations (5.8), (5.18), and (5.19) allow us to study the dependence of $\widetilde{a}_{1}$ and $\widetilde{b}_{1}$ on the aspect ratio of the particle. While one expects that the leading particle amplitude $\widetilde{a}_{1}$ stays positive and for fixed sphere diameter $L$ monotonically decreases from its value for touching spheres towards zero as $\alpha$ increases from 0 to $2 \pi$, the anisotropy amplitude $\tilde{b}_{1}$ changes sign from positive to negative values as the particle shape changes from prolate dumbbells with $\alpha<\pi$ as in the upper part of Fig. 3 to oblate lenses with $\pi<\alpha$ as in the lower part.

For a dumbbell or lens of nearly isotropic shape with $\alpha$ near $\pi$, i.e., with small center to center distance $\left|d_{c c}\right|$ $\rightarrow|\pi-\alpha| L / 2$ between the two spheres,

$$
\tilde{a}_{1} B_{\varphi}^{1 / 2} /\left(\mathcal{A}_{\varphi}^{(+)} \mathcal{D}^{x_{\varphi}}\right) \rightarrow 1+\left[\frac{1}{8} ; \frac{2}{3}\right]\left(1-\frac{\alpha}{\pi}\right)+O\left((\pi-\alpha)^{2}\right)
$$

and

$$
\widetilde{b}_{1} B_{\varphi}^{1 / 2} /\left(\mathcal{A}_{\varphi}^{(+)} \mathcal{D}^{x_{\varphi}+2}\right) \rightarrow\left[\frac{2}{9} ; \frac{1}{6}\right]\left(1-\frac{\alpha}{\pi}\right)+O\left((\pi-\alpha)^{2}\right)
$$

for $[d=2 ; d=4]$. Here we have replaced the parameter $m$ in Eqs. (5.8) and (5.19) by $4|\pi-\alpha| /(3 \pi)$ to first order in $\pi$ $-\alpha$. In Eqs. (5.20) and (5.21) $\mathcal{D}$ can be replaced by the sphere diameter $L$, since the difference is second order in $\pi-\alpha$.

For the case of two weakly overlapping spheres with a lens of overlap (shown by short dashes in the upper right of Fig. 3) of width $L \alpha^{2} / 8$ much smaller than $L$ and $\mathcal{D}$,

$$
\widetilde{a}_{1} B_{\varphi}^{1 / 2} /\left(\mathcal{A}_{\varphi}^{(+)} L^{x_{\varphi}}\right) \rightarrow a\left(1-\frac{\alpha^{2}}{8} a^{\prime}\right)
$$

with

$$
\begin{aligned}
& a=\left[\left(\frac{\pi}{2}\right)^{1 / 8} ; \frac{K}{\sqrt{2}}\right]=[1.058 ; 1.311], \\
& a^{\prime}=\left[\frac{1}{24} ; \frac{1}{3}-\frac{K-\bar{K}}{K^{3}}\right]=[0.042 ; 0.175],
\end{aligned}
$$

and

$$
\tilde{b}_{1} B_{\varphi}^{1 / 2} /\left(\mathcal{A}_{\varphi}^{(+)} L^{x_{\varphi}+2}\right) \rightarrow b\left(1-\frac{\alpha^{2}}{8} b^{\prime}\right)
$$

with

$$
\begin{aligned}
& b=\left[\frac{1}{9}\left(\frac{\pi}{2}\right)^{17 / 8} ; \frac{K^{3}}{16 \sqrt{2}}\right]=[0.290 ; 0.282], \\
& b^{\prime}=\left[\frac{17}{24}+\frac{8}{\pi^{2}} ; 1+\frac{K+3 \bar{K}}{K^{3}}\right]=[0.708 ; 0.811]
\end{aligned}
$$


for $[d=2 ; d=4]$. Here $K=K(m=1 / 2)=1.854$ and $\bar{K}$ $=(d K / d m)_{m=1 / 2}=0.847$ are the complete elliptic integral and its derivative at $m=1 / 2$, and we have used that for $\alpha$ $\rightarrow 0$ the quantities $1-2 m$ and $\mathcal{D}(1-2 m)^{-1 / 2}$ tend to $\alpha^{2} /\left(4 K^{2}\right)$ and $L K\left[1-\left(1 / 3+\bar{K} / K^{3}\right) \alpha^{2} / 8\right]$, respectively.

For a disk of diameter $\mathcal{D}$ (which according to the lower right part of Fig. 3 is the limit of the lens for $\alpha \rightarrow 2 \pi$ ),

$$
\begin{aligned}
\tilde{a}_{1} B_{\varphi}^{1 / 2} /\left(\mathcal{A}_{\varphi}^{(+)} \mathcal{D}^{x_{\varphi}}\right) & \rightarrow\left[2^{-1 / 8} ;\left(1+m_{2 \pi}\right)^{-1 / 2}\right] \\
& =[0.917 ; 0.740]
\end{aligned}
$$

and

$$
\begin{aligned}
\tilde{b}_{1} B_{\varphi}^{1 / 2} /\left(\mathcal{A}_{\varphi}^{(+)} \mathcal{D}^{x_{\varphi}+2}\right) & \rightarrow-\left[\frac{2^{-1 / 8}}{12} ; \frac{m_{2 \pi} / 8}{\left(1+m_{2 \pi}\right)^{3 / 2}}\right] \\
& =-[0.0764 ; 0.0419]
\end{aligned}
$$

for $[d=2 ; d=4]$. Here the internal dimension of the disk is $d-1$, i.e., in $d=2$ the disk is a line of length $\mathcal{D}$.

Due to Eq. (5.20) the ratio $\widetilde{a}_{1} /\left(\widetilde{a}_{1}\right)_{\text {sphere }}$ of the leading amplitudes of a disk of diameter $\mathcal{D}$ and a sphere with diameter $2 R$ is, apart from a factor $[\mathcal{D} /(2 R)]^{x}$, also given by the right hand side of Eq. (5.26). Of course, $\widetilde{a}_{1}$ is smaller for the disk than for the sphere with the same diameter.

It is instructive to compare the leading anisotropy amplitude $\widetilde{b}_{1}$ of the disk with that of the dumbbell in Fig. 1(b) of two touching spheres, each of diameter 2R. From Eqs. (5.24) and (5.27) one finds a ratio

$$
\begin{aligned}
\frac{\tilde{b}_{1}}{\left(\tilde{b}_{1}\right)_{\mathrm{DB}}}\left(\frac{4 R}{\mathcal{D}}\right)^{x_{\varphi}+2} & \rightarrow-\left[3\left(\frac{2}{\pi}\right)^{17 / 8} ; \frac{16 \sqrt{2} m_{2 \pi}}{\left(1+m_{2 \pi}\right)^{3 / 2}[K(1 / 2)]^{3}}\right] \\
& =-[1.149 ; 1.188]
\end{aligned}
$$

which, apart from the sign, is close to 1 . Thus in both $d$ $=2$ and $d=4$ for a disk with diameter $\mathcal{D}$ and a dumbbell with length $4 R=\mathcal{D}$ nearly the same free energy is required to turn the particle from a perpendicular to parallel orientation with respect to a neighboring wall with the same preference for one of the two solvent phases.

\section{SUMMARY AND CONCLUDING REMARKS}

The behavior of anisotropic colloidal particles in a critical fluid mixture is studied in this paper by methods of field theory. The angle dependent particle-wall and particleparticle interactions induced by the critical solvent are investigated for mesoscopic particles which are small compared to the correlation length and interparticle distances.

The discussion is based on a "small particle operator expansion" 7-10 in an Ising-type field theory, extended to the case of anisotropic particles. The operators and their weights are fully determined by the size, shape, and boundary condition of the small mesoscopic particle. For a given particle the leading behavior of a large variety of anisotropic properties, such as the interactions mentioned above, multiparticle interactions, and profiles and multipoint correlation functions of densities, is described by only a few operator weights.

The main emphasis is on dumbbell-shaped dimer particles composed of two colloidal spheres that touch or over- lap. These have been synthesized recently ${ }^{20-22}$ with a controlled size and aspect ratio. On the theoretical side dumbbells are particularly convenient anisotropic shapes, since they can be mapped by an angle preserving (conformal) transformation onto the simple geometries of two parallel planes or two half planes forming a wedge.

The concern of the paper is twofold.

(i) The "small particle expansion" is an important and versatile tool based on a supposedly exact operator relationship which is similar in spirit to the well known "operator product expansion" for field theories in unbounded space. However, in contrast to the latter, no general proof has been given for the former. Thus we have placed a strong emphasis on checking it for consistency in various situations.

(ii) In order to make semiquantitative predictions for the observable angle-dependent interactions, we obtained explicit estimates for the operator weights.

Here is a summary of the main results:

(1) For the three dumbbells of Fig. 1 the leading isotropic and anisotropic operators were identified, see Eqs. (2.1)(2.8). These are the operators of lowest scaling dimension which are consistent with the surface universality class of the particle (preserving or breaking the up-down symmetry of the order parameter) and its spatial symmetry (axis of rotation, center of inversion). Operators odd in the order parameter symmetry only appear if the symmetry is broken, as in Figs. 1(b,c). The anisotropic operators are also absent for an isotropic spherical particle. Note that the symmetry preserving (ord, ord) dumbbell of Fig. 1(a) with the axis along the unit vector $\vec{\varrho}$ has the same symmetries as the product $\mathcal{O}(\mathbf{r}$ $-\mathbf{s} / 2) \mathcal{O}(\mathbf{r}+s / 2)$ of two identical operators (such as two order parameters $\varphi$ or two energy densities $\epsilon$ ) separated by the short distance vector $\mathbf{s}$. Therefore, the same leading anisotropic operators $\partial_{\|}^{2} \epsilon=\Sigma \varrho_{\mu} \varrho_{\nu} \partial_{\mu} \partial_{\nu} \epsilon$ and $T_{\|,\|}=\Sigma \varrho_{\mu} \varrho_{\nu} T_{\mu \nu}$, with $T_{\mu \nu}$ the stress tensor, appear in the dumbbell and operator product expansions, see Eq. (2.5) with Eqs. (2.39) and (A1). The role of the axis vector $\vec{\varrho}$ in the former is played by the direction $\mathbf{s} / s$ in the latter. Since dumbbells of two penetrating spheres, lenses, or ellipsoids of revolution with (ord) and $(+)$ surfaces have the same symmetries as the dumbbells in Figs. 1(a) and 1(b), we also expect the operators of Eqs. (2.1)-(2.6) to appear in these cases, but with different weights.

(2) The weights of the even, odd, isotropic, and anisotropic operators for the dumbbells in Fig. 1 can be expressed in terms of critical density profiles and their derivatives on the midplane of a film bounded by two parallel plates, see Eqs. (2.9)-(2.19). The reason why these weights describe the effect of the particle not only on density profiles but also on multipoint correlation functions can be easily understood for two-point averages. The conformal inversion (2.25) about the dumbbell center maps two points far from the dumbbell onto two points close to a midpoint between the plates. Thus the operator product expansion can be applied to the corresponding two-point average in the film, leading to a linear combination of film density profiles and their derivatives, see Sec. II C 2.

(3) In a similar way the weights for the more general 
dumbbells and lenses of Fig. 3 can be expressed in terms of density profiles near the midplane of a wedge, as explained in Sec. V. The results depend on the aspect ratio of the particle determined by the angle $\alpha$ of intersection between the two surfaces of sphere segments, as shown on the right hand sides of Fig. 3. This angle is equal to the opening angle of the wedge.

(4) Explicit results in spatial dimensions $d$ near 4 and in $d=2$ for profiles and multipoint averages in film and wedge geometries determine corresponding results for dumbbells and allow one to verify the general structure of and to determine the weights in the particle expansions. For $d / 4$ the behavior reduces to that of the Gaussian model and of meanfield theory, respectively, in the symmetry preserving and symmetry breaking cases of Fig. 1(a) and Figs. 1(b,c). The agreement found in Secs. III B and III A with the small particle expansion arises in an interesting, nontrivial way. For example, it is shown in Eqs. (3.22)-(3.30) of Sec. III A 2 and in section 1 of Appendix B that a higher loop calculation of the bulk three-point amplitude $C_{\varphi \varphi \lambda}$ is required in order to reproduce, from the particle expansion, the mean field expression (3.30) for the order parameter two-point average in the presence of a $(+,+)$ or $(-,+)$ dumbbell. Equation (3.30) involves a cooperation of the four operators $\left(\Delta, \partial_{\|}^{2}\right) \epsilon$, $T_{\|,\|}$, and $\lambda$. In higher order in the $\varepsilon$ expansion, the small particle operator expansion helps disentangle contributions of order $x_{\epsilon}+2, d$, and $d+\omega$ in the particle size, which become degenerate and equal to 4 as $d / 4$. Here $\omega$ is the correction to scaling exponent. A check beyond mean field theory of the operator product expansion near a symmetry breaking wall is presented in Sec. 2 of Appendix B. For $d$ $=2$ the results of profiles and two- and three-point functions presented in Sec. III C and Appendix D all follow the predictions of the small dumbbell expansion, see Eqs. (3.54)(3.58) and Eqs. (3.65) and (3.66). In Eqs. (3.59)-(3.64) we identify the even and isotropic stress tensor product $T \bar{T}$ in the small particle expansion of an isotropic particle in $d$ $=2$. For the dumbbells in Fig. 1 the weights in $d / 4$ and $d=2$ are given in Eqs. (3.10)-(3.17), (3.31)-(3.39), and (3.49)-(3.53). For the more general dumbbell and lens shapes of Fig. 3 explicit results are presented for arbitrary aspect ratios and shown to be consistent with the particle expansion. See Eq. (5.14) with Eqs. (5.6), (5.7), and (5.17)(5.28).

(5) Results for dumbbell and ellipsoid shapes are compared. We consider the smallest prolate ellipsoid that circumscribes a dumbbell of the shape shown in Fig. 1. The qualitative observation that the dumbbell is smaller and more anisotropic than the ellipsoid is made quantitative by calculating in $d=3$, for the simple case of a Gaussian model and symmetry preserving surfaces, the leading isotropic and anisotropic weights of the two particles, see Eqs. (3.42) and (3.43). Small dumbbell and operator product expansions in the Gaussian model are checked in Appendix C.

(6) Since the operator representation of a particle applies with the same weights in the presence of a distant wall or another particle, it can be used to evaluate particle-wall and particle-particle interaction free energies induced by the critical solvent. For anisotropic particles these depend on the mutual orientations. The results for symmetry breaking dumbbells near $a$ wall at the critical composition and $T$ $\geqslant T_{c}$ are given in Eqs. (4.2) and (4.3), with the orientations of lowest free energy shown in Fig. 2. The interactions have been checked for the case of $T=T_{c}$ in which the dumbbell near wall problem can be mapped onto the problem of a sphere between two parallel walls, and either the dumbbell or sphere expansion can be used. Equation (4.2) not only applies to the dumbbell of two touching spheres in Fig. 1(b) but also to the more general dumbbell and lens shapes of Fig. 3 , with the amplitudes $\widetilde{a}_{1}$ and $\widetilde{b}_{1}$ given by Eqs. (5.18) and (5.19). As expected, the anisotropy amplitude $\tilde{b}_{1}$ changes sign as the shape changes from a dumbbell to a lens, see Eq. (5.21), and in both cases the particle orientation perpendicular to the wall has the lowest free energy if particle and wall prefer the same component of the mixture. Results for the interaction between two particles are given in Eqs. (4.10) and (4.11). The isotropic and anisotropic interactions right at the critical point depend on the interparticle distance according to power laws. The free energies required to turn the $(+$, $+)$ or $(-,+)$ dumbbells of Figs. 1(b,c) near a wall from the favorable to the unfavorable orientation show an interesting temperature dependence with a maximum above $T_{c}$. The free energy cost right at the critical point is compared with the corresponding energy change $\Delta E$ from the van der Waals interaction in the paragraph preceding Eq. (4.9).

It would be interesting to extend this work in the following directions:

(a) Studying the solvent-induced interactions of anisotropic particles with a wall or other particles in the neighborhood of the critical point of the mixture, i.e., as a function of both relevant thermodynamic variables temperature and composition.

(b) Determining the full crossover from small to large size of the anisotropic particle. As a first step a discussion within Landau theory ${ }^{10}$ would be very valuable.

(c) Investigating the interactions in other types of solvents such as polymer solutions or ${ }^{4} \mathrm{He}$.

\section{ACKNOWLEDGMENTS}

The author thanks T.W. Burkhardt, M. Krech, and M. Laessig for useful discussions.

\section{APPENDIX A: EXPRESSIONS FROM CONFORMAL FIELD THEORY}

Here we give the forms of the differential operators $\mathcal{D}$ in the operator product expansions (2.39) and (2.47). The contribution to the product $\mathcal{O}_{1}\left(\hat{\mathbf{r}}_{1}\right) \mathcal{O}_{2}\left(\hat{\mathbf{r}}_{2}\right)$ of two scalar operators $\mathcal{O}_{1}, \mathcal{O}_{2}$ with scaling dimensions $x_{1}, x_{2}$ from a scalar operator $\mathcal{O}_{3}$ with dimension $x_{3}$ and from its derivatives is $\operatorname{given}^{31}$ by $\left[\mathcal{D}_{x_{1}-x_{2}, x_{3}}\left(\mathbf{s}, \partial_{\hat{\mathbf{r}}}\right) \mathcal{O}_{3}(\hat{\mathbf{r}})\right] C_{\mathcal{O}_{1} \mathcal{O}_{2} \mathcal{O}_{3}} /\left(B_{\mathcal{O}_{3}} s^{x_{1}+x_{2}-x_{3}}\right)$, with $\mathbf{s}$ from Eq. (2.40), 


$$
\begin{aligned}
\mathcal{D}_{x_{1}-x_{2}, x_{3}}\left(\mathbf{s}, \partial_{\hat{\mathbf{r}}}\right)= & 1+\frac{1}{8} \frac{x_{3}^{2}-\left(x_{1}-x_{2}\right)^{2}}{x_{3}\left(x_{3}+1\right)} \\
& \times\left(-\frac{1}{2 x_{3}-d+2} s^{2} \Delta_{\hat{\mathbf{r}}}\right. \\
& \left.+\frac{1}{x_{3}} \sum_{\mu, \nu} s_{\mu} s_{\nu} \partial_{\hat{r}_{\mu}} \partial_{\hat{r}_{\nu}}\right)+O\left(s^{3}\right)
\end{aligned}
$$

and

$$
\hat{\mathbf{r}}=\frac{1}{2}\left(\hat{\mathbf{r}}_{1}+\hat{\mathbf{r}}_{2}\right)+\frac{x_{1}-x_{2}}{2 x_{3}}\left(\hat{\mathbf{r}}_{1}-\hat{\mathbf{r}}_{2}\right) .
$$

The differential operator is chosen so that $\mathcal{D}_{x_{1}-x_{2}, x_{3}}\left(\mathbf{s}, \partial_{\hat{\mathbf{r}}}\right)\left|\hat{\mathbf{r}}_{3}-\hat{\mathbf{r}}\right|^{-2 x_{3}}$ equals $1 /\left(\hat{r}_{23}^{x_{2}+x_{3}-x_{1}} \hat{r}_{31}^{x_{3}+x_{1}-x_{2}}\right)$. Thus the bulk three-point function

$$
\begin{aligned}
\left\langle\mathcal{O}_{1}\left(\mathbf{r}_{1}\right) \mathcal{O}_{2}\left(\mathbf{r}_{2}\right) \mathcal{O}_{3}\left(\mathbf{r}_{3}\right)\right\rangle_{\text {bulk }} & C_{\mathcal{O}_{1} \mathcal{O}_{2} \mathcal{O}_{3}} \\
= & \frac{r_{12}^{x_{1}+x_{2}-x_{3}} r_{23}^{x_{2}+x_{3}-x_{1}} r_{31}^{x_{3}+x_{1}-x_{2}}}{}
\end{aligned}
$$

is correctly reproduced by the OPE. Due to the choice of $\hat{\mathbf{r}}$ in Eq. (A2), $\mathcal{D}$ contains no first derivative.

From Eq. (A3) we obtain

$$
\begin{aligned}
\left\langle\mathcal{O}\left(\mathbf{r}_{1}\right) \mathcal{O}\left(\mathbf{r}_{2}\right) \partial_{\|}^{2} \epsilon\right\rangle_{\text {bulk }}= & \left\langle\mathcal{O}\left(\mathbf{r}_{1}\right) \mathcal{O}\left(\mathbf{r}_{2}\right) \epsilon\right\rangle_{\text {bulk }}\left\{2 x_{\epsilon}^{2} U_{12}\right. \\
& \left.+x_{\epsilon}\left(x_{\epsilon}+2\right) U_{+}-x_{\epsilon}\left(\frac{1}{r_{1}^{2}}+\frac{1}{r_{2}^{2}}\right)\right\}, \\
\left\langle\mathcal{O}\left(\mathbf{r}_{1}\right) \mathcal{O}\left(\mathbf{r}_{2}\right)\left[a_{2} \Delta \epsilon+b_{1} \partial_{\|}^{2} \epsilon\right]\right\rangle_{\text {bulk }} / B_{\mathcal{O}} & \\
= & \frac{1}{2}\left(\frac{L}{2}\right)^{x \epsilon^{+2}} \mathcal{E}^{\prime \prime}(0)\left\{U_{+}+2 U_{12}+\frac{1}{x_{\epsilon}+1}\right. \\
& \left.\times\left[\left(-\frac{x_{\epsilon}}{2 x_{\epsilon}+2-d} I\right)+U_{+}-2 U_{12}\right]\right\} \\
& \times \frac{\left\langle\mathcal{O}\left(\mathbf{r}_{1}\right) \mathcal{O}\left(\mathbf{r}_{2}\right) \epsilon\right\rangle_{\text {bulk }}}{B_{\mathcal{O}} B_{\epsilon}^{1 / 2}},
\end{aligned}
$$

for the derivatives of bulk three-point functions needed in Eq. (2.46) for any of the dumbbells in Fig. 1, and

$$
\begin{aligned}
& \left\langle\varphi\left(\mathbf{r}_{1}\right) \epsilon\left(\mathbf{r}_{2}\right) \partial_{\|}^{2} \varphi\right\rangle_{\text {bulk }} \\
& =\left\langle\varphi\left(\mathbf{r}_{1}\right) \epsilon\left(\mathbf{r}_{2}\right) \varphi\right\rangle_{\text {bulk }}\left\{-2 x x_{\epsilon} U_{12}-x(2-x) U_{1}\right. \\
& \left.\quad+x_{\epsilon}\left(x_{\epsilon}+2\right) U_{2}+\frac{x}{r_{1}^{2}}-\frac{x_{\epsilon}}{r_{2}^{2}}\right\},
\end{aligned}
$$

$$
\begin{aligned}
\left\langle\varphi\left(\mathbf{r}_{1}\right)\right. & \left.\epsilon\left(\mathbf{r}_{2}\right)\left[\tilde{a}_{2} \Delta \varphi+\tilde{b}_{1} \partial_{\|}^{2} \varphi\right]\right\rangle_{\text {bulk }} /\left(B_{\varphi} B\right)^{1 / 2} \\
= & \frac{1}{2}\left(\frac{L}{2}\right)^{x_{\varphi}+2} \frac{\mathcal{F}_{++}^{\prime \prime}(0)}{x_{\varphi}\left(x_{\varphi}+1\right)}\left\{\frac{x x_{\epsilon}}{2 x_{\varphi}+2-d} I-2 x x_{\epsilon} U_{12}\right. \\
& \left.-x(2-x) U_{1}+x_{\epsilon}\left(x_{\epsilon}+2\right) U_{2}\right\} \frac{\left\langle\varphi\left(\mathbf{r}_{1}\right) \epsilon\left(\mathbf{r}_{2}\right) \varphi\right\rangle_{\text {bulk }}}{B_{\varphi} B_{\epsilon}^{1 / 2}}
\end{aligned}
$$

in Eq. (2.50) for the $(+,+)$ dumbbell. In Eqs. (A4)-(A7),

$$
\begin{aligned}
& I=\frac{r_{12}^{2}}{r_{1}^{2} r_{2}^{2}}, \quad U_{1}=\frac{r_{1 \|}^{2}}{r_{1}^{4}}, \quad U_{2}=\frac{r_{2 \|}^{2}}{r_{2}^{4}}, U_{+}=U_{1}+U_{2}, \\
& U_{12}=\frac{r_{1 \|} r_{2 \|}}{r_{1}^{2} r_{2}^{2}}, \quad x=x_{\epsilon}-2 x_{\varphi} .
\end{aligned}
$$

Note that $U_{+} \pm 2 U_{12}$ equals $\left[\left(r_{1 \|} / r_{1}^{2}\right) \pm\left(r_{2 \|} / r_{2}^{2}\right)\right]^{2}=\left[\hat{z}_{1}\right.$ $\left.\pm \hat{z}_{2}\right]^{2} / L^{4}$.

We also give the form of the three-point function with one stress tensor ${ }^{24}$

$$
\begin{aligned}
\left\langle\mathcal{O}\left(\mathbf{r}_{1}\right) \mathcal{O}\left(\mathbf{r}_{2}\right) T_{k l}\left(\mathbf{r}_{3}\right)\right\rangle_{\text {bulk }}= & \frac{C_{\mathcal{O O T}}}{\left(r_{13} r_{23}\right)^{d} r_{12}^{2 x_{\mathcal{O}}-d}} \\
& \times\left[\frac{\left(X_{12}\right)_{k}\left(X_{12}\right)_{l}}{\left|X_{12}\right|^{2}}-\frac{1}{d} \delta_{k l}\right],
\end{aligned}
$$

where

$$
\mathbf{X}_{12}=\frac{\mathbf{r}_{13}}{r_{13}^{2}}-\frac{\mathbf{r}_{23}}{r_{23}^{2}}
$$

and $x_{\mathcal{O}}$ is the scaling dimension of the scalar operator $\mathcal{O}$. In particular,

$$
\begin{aligned}
\left\langle\mathcal{O}\left(\mathbf{r}_{1}\right) \mathcal{O}\left(\mathbf{r}_{2}\right) T_{\|,\|}(0)\right\rangle_{\text {bulk }}= & \frac{-C_{\mathcal{O O T}}}{\left(r_{1} r_{2}\right)^{d-2} r_{12}^{2 x_{\mathcal{O}^{-d+2}}}} \\
& \times\left[\frac{1}{d} I-U_{+}+2 U_{12}\right] .
\end{aligned}
$$

\section{APPENDIX B: LOOP EXPANSION RESULTS NEAR FOUR DIMENSIONS}

\section{Universal three-point amplitude of $\langle\varphi \varphi \lambda\rangle$}

The leading even operator $\lambda$ beyond the energy density in the critical $\Phi^{4}$ Ising model with Hamiltonian

$$
\mathcal{H}=\int d \mathbf{r}^{\prime}\left\{\frac{1}{2}\left[\nabla_{\mathbf{r}^{\prime}} \Phi\left(\mathbf{r}^{\prime}\right)\right]^{2}+u \frac{1}{4 !}\left[\Phi\left(\mathbf{r}^{\prime}\right)\right]^{4}\right\}
$$

can be constructed from the renormalized composite operators $\left[\Phi^{4}\right]_{R}(\mathbf{r})$ and $\left[\Phi^{2}\right]_{R}(\mathbf{r})$, which are related to their bare counterparts $[\Phi(\mathbf{r})]^{4}$ and $[\Phi(\mathbf{r})]^{2}$ by $^{28}$

$$
\left(\begin{array}{c}
u \frac{1}{4 !} \Phi^{4} \\
-\Delta_{\mathbf{r}} \Phi^{2}
\end{array}\right)=\mathcal{Z}\left(\begin{array}{c}
U \frac{1}{4 !}\left[\Phi^{4}\right]_{\mathrm{R}} \\
-\Delta_{\mathbf{r}}\left[\Phi^{2}\right]_{\mathrm{R}}
\end{array}\right), \quad \mathcal{Z}=\left(\begin{array}{cc}
Z_{44}, & Z_{42} \\
0, & Z_{22}
\end{array}\right)
$$


with a $2 \times 2$ renormalization matrix $\mathcal{Z}$. As in Refs. 13 and 28, we use dimensional regularization. The renormalization factors $Z_{u}$ and $Z_{\Phi}$ in

$$
u=U Z_{u}\left(u_{\mathrm{R}}, \varepsilon\right), \quad U=16 \pi^{2} f \mu^{\varepsilon} u_{\mathrm{R}},
$$

and

$$
\Phi=Z_{\Phi}^{1 / 2} \Phi_{\mathrm{R}},
$$

which relate the bare and renormalized coupling constants $u$ and $u_{\mathrm{R}}$ and order parameter fields $\Phi$ and $\Phi_{\mathrm{R}}$ as well as the renormalization matrix $\mathcal{Z}$ in Eq. (B2), only depend on $\varepsilon$ via pole terms ("minimal subtraction"). In Eq. (B3) $\mu$ is an arbitrary inverse length scale and $f=1+\varepsilon f_{1}+\varepsilon^{2} f_{2}+\cdots$ is an $\varepsilon$-dependent prefactor.

Since the energy density $\propto-\Phi^{2}$ is multiplicatively renormalized via $\Phi^{2}=Z_{t}^{-1}\left[\Phi^{2}\right]_{\mathrm{R}}$, the lower nondiagonal entry of $\mathcal{Z}$ in Eq. (B2) vanishes, and $Z_{22}$ equals $Z_{t}^{-1}$. However, inserting $\Phi^{4}(\mathbf{r})$ into a $\Phi_{R}\left(\mathbf{r}_{1}\right) \Phi_{R}\left(\mathbf{r}_{2}\right)$ average also generates $\varepsilon$ pole-terms with the $\mathbf{r}, \mathbf{r}_{1}, \mathbf{r}_{2}$ dependence of $\left\langle\Delta \Phi^{2} \Phi_{\mathrm{R}} \Phi_{\mathrm{R}}\right\rangle$, and the mixing element

$$
Z_{42}=-\frac{\theta\left(\varepsilon, u_{\mathrm{R}}\right)}{\varepsilon}, \quad \theta=\theta_{1}\left(u_{\mathrm{R}}\right)+\frac{1}{\varepsilon} \theta_{2}\left(u_{\mathrm{R}}\right)+\cdots
$$

is nonvanishing, with ${ }^{28}$

$$
\theta_{1}=\frac{u_{\mathrm{R}}^{3}}{36}+O\left(u_{\mathrm{R}}^{4}\right), \quad \theta_{2}=O\left(u_{\mathrm{R}}^{4}\right)
$$

The upper diagonal element of $\mathcal{Z}$ is ${ }^{28}$

$$
Z_{44}=-\beta_{u} /\left(u_{\mathrm{R}} \varepsilon\right)=1-\frac{1}{\varepsilon} \bar{\beta} / u_{\mathrm{R}},
$$

with the usual $\beta$ function

$$
\beta_{u}\left(u_{\mathrm{R}}, \varepsilon\right)=\mu \frac{D u_{\mathrm{R}}}{D \mu}=-\varepsilon u_{\mathrm{R}}+\bar{\beta}\left(u_{\mathrm{R}}\right), \bar{\beta}=3 u_{\mathrm{R}}^{2}+O\left(u_{\mathrm{R}}^{3}\right),
$$

where the $\mu$-variation $D \mu$ is at fixed bare coupling $u$. The pole subtraction effected by $\mathcal{Z}$ in Eq. (B2) guarantees that the dimensionless functions $M_{4}, M_{2}$ in the averages

$$
\begin{aligned}
& \left\langle U \frac{1}{4 !}\left[\Phi^{4}\right]_{\mathrm{R}}(\mathbf{r}) \Pi_{i} \Phi_{\mathrm{R}}\left(\mathbf{r}_{i}\right)\right\rangle \\
& \quad=\mu^{d+N(d-2) / 2} M_{4}^{(N)}\left(\mu \mathbf{r},\left\{\mu \mathbf{r}_{i}\right\} ; u_{\mathrm{R}}, \varepsilon\right)
\end{aligned}
$$

and

$$
\begin{aligned}
& \left\langle\left[\Phi^{2}\right]_{\mathrm{R}}(\mathbf{r}) \Pi_{i} \Phi_{\mathrm{R}}\left(\mathbf{r}_{i}\right)\right\rangle \\
& \quad=\mu^{d-2+N(d-2) / 2} M_{2}^{(N)}\left(\mu \mathbf{r},\left\{\mu \mathbf{r}_{i}\right\} ; u_{\mathrm{R}}, \varepsilon\right)
\end{aligned}
$$

with $i=1,2, \ldots, N$ are finite for $\varepsilon \rightarrow 0$, order by order in $u_{\mathrm{R}}$.

Since the dependence on bare variables of the averages of bare operators does not involve $\mu$, Eq. (B2) implies the two renormalization-group equations

$$
\left\{1\left(\mathcal{D}_{N}+d\right)+\alpha\right\}\left(\begin{array}{c}
M_{4}^{(N)} \\
-\Delta_{\mu \mathbf{r}} M_{2}^{(N)}
\end{array}\right)=0,
$$

where 1 denotes the $2 \times 2$ unit matrix,

$$
\mathcal{D}_{N}=\mu \partial_{\mu}+\beta_{u} \partial_{u_{\mathrm{R}}}+\frac{N}{2}\left(d-2+\eta_{\Phi}\right), \quad \eta_{\Phi}=\frac{D \ln Z_{\Phi}}{D \ln \mu},
$$

and

$$
\alpha=\mathcal{Z}^{-1} \mu \frac{D}{D \mu} \mathcal{Z}=\left(\begin{array}{cc}
\alpha_{44}, & \alpha_{42} \\
0, & \alpha_{22}
\end{array}\right) .
$$

Here

$$
\begin{aligned}
& \alpha_{44}=u_{\mathrm{R}} \partial_{u_{\mathrm{R}}}\left(\bar{\beta} / u_{\mathrm{R}}\right)=\partial_{u_{\mathrm{R}}} \beta_{u}-\beta_{u} / u_{\mathrm{R}}, \\
& \alpha_{42}=u_{\mathrm{R}} \partial_{u_{\mathrm{R}}} \theta_{1}\left(u_{\mathrm{R}}\right),
\end{aligned}
$$

and

$$
\alpha_{22}=Z_{t} \mu \frac{D}{D \mu} Z_{t}^{-1}=-\eta_{t}=u_{\mathrm{R}}+O\left(u_{\mathrm{R}}^{2}\right) .
$$

At the infrared stable fixed point $u_{\mathrm{R}}=u_{\mathrm{R}}^{*}=\varepsilon / 3+O\left(\varepsilon^{2}\right)$, where $\beta_{u}$ vanishes,

$$
\alpha_{44} \rightarrow \alpha_{44}^{*}=\left(\partial_{u_{\mathrm{R}}} \beta_{u}\right)^{*}=\omega=\varepsilon+O\left(\varepsilon^{2}\right)
$$

is the correction to scaling exponent, and

$$
\alpha_{22} \rightarrow \alpha_{22}^{*}=-\eta_{t}^{*}=x_{\epsilon}+2-d=\frac{\varepsilon}{3}+O\left(\varepsilon^{2}\right)
$$

is the anomalous dimension of the energy density.

From the matrix equation (B11) one concludes that

$$
\left[\mathcal{D}_{N}+d+\alpha_{44}\right]\left\{M_{4}^{(N)}+\frac{\alpha_{42}}{\alpha_{44}-\alpha_{22}}\left(-\Delta_{\mu \mathbf{r}}\right) M_{2}^{(N)}\right\}=0,
$$

and from Eqs. (B9) and (B10) that the scalar operator

$$
\lambda(\mathbf{r})=U \frac{1}{4 !}\left[\Phi^{4}\right]_{\mathrm{R}}(\mathbf{r})+\frac{\alpha_{42}}{\alpha_{44}-\alpha_{22}}\left(-\Delta_{\mathbf{r}}\right)\left[\Phi^{2}\right]_{\mathrm{R}}(\mathbf{r})
$$

with the (naive) inverse length dimension $d$ has scaling dimension $d+\omega$. Thus, one expects that its three-point function with two $\Phi_{\mathrm{R}}$ 's approaches the form (A3),

$$
\left\langle\Phi_{\mathrm{R}}\left(\mathbf{r}_{1}\right) \Phi_{\mathrm{R}}\left(\mathbf{r}_{2}\right) \lambda(\mathbf{r}=0)\right\rangle=\frac{C_{\Phi_{\mathrm{R}} \Phi_{\mathrm{R}} \lambda}}{r_{12}^{2 x_{\varphi}-x_{\lambda}}\left(r_{1} r_{2}\right)^{x_{\lambda}}}
$$

in the long distance limit. Using the result

$$
M_{4}^{(2)}=\frac{u_{\mathrm{R}}^{2}}{48 \pi^{4}} \frac{1}{\mu^{6}} \frac{1}{\left(r_{1} r_{2}\right)^{2}}\left(\frac{1}{r_{1}^{2}}+\frac{1}{r_{2}^{2}}\right)+O\left(u_{\mathrm{R}}^{2} \varepsilon, u_{\mathrm{R}}^{3}\right)
$$

of a calculation of $\left\langle\Phi^{4} \Phi \Phi\right\rangle$ to first order in $u$ and the relation

$$
\begin{aligned}
\frac{\alpha_{42}}{\alpha_{44}-\alpha_{22}}\left(-\Delta_{\mu \mathbf{r}}\right) M_{2}^{(2)}= & \frac{u_{\mathrm{R}}^{2}}{24}(-) \frac{1}{\pi^{4}} \frac{1}{\mu^{6}} \frac{\mathbf{r}_{1} \mathbf{r}_{2}}{\left(r_{1} r_{2}\right)^{4}} \\
& +O\left(u_{\mathrm{R}}^{2} \varepsilon, u_{\mathrm{R}}^{3}\right)
\end{aligned}
$$

following from Eq. (B15) with Eq. (B6), Eq. (B14) with Eq. (B8), and Eq. (B16), together with the $u=0$ expression of $\left\langle\Phi^{2} \Phi \Phi\right\rangle$, one concludes that the left hand side of Eq. (B21) has an $\mathbf{r}_{1}, \mathbf{r}_{2}$ dependence which is compatible with its right hand side, and $C_{\Phi_{\mathrm{R}} \Phi_{\mathrm{R}} \lambda}$ is given by $\left(u_{\mathrm{R}}^{*}\right)^{2} /\left(48 \pi^{4}\right)$ in leading 
order in $\varepsilon$. With the leading order expression $B_{\lambda}$ $=\left[u_{\mathrm{R}}^{*} /\left(2 \sqrt{6} \pi^{2}\right)\right]^{2}$ for the amplitude of the two-point function $\langle\lambda(\mathbf{r}) \lambda(0)\rangle$, which follows from evaluating $\left\langle\Phi^{4}(\mathbf{r}) \Phi^{4}(0)\right\rangle$ for $u=0$ from Wick's theorem, and with $B_{\Phi_{R}}=(2 \pi)^{-2}$ for the amplitude of $\left\langle\Phi_{R}(\mathbf{r}) \Phi_{R}(0)\right\rangle$, one finally obtains, for the universal three-point amplitude

$$
\frac{C_{\varphi \varphi \lambda}}{B_{\varphi} B_{\lambda}^{1 / 2}}=\frac{C_{\Phi_{\mathrm{R}} \Phi_{\mathrm{R}} \lambda}}{B_{\Phi_{\mathrm{R}}} B_{\lambda}^{1 / 2}},
$$

the expression $\varepsilon /(3 \sqrt{6})$ given in Eq. (3.24).

\section{Short distance expansion of $\langle\varphi \varphi\rangle$ near a symmetry breaking wall}

Here we study the short distance behavior of the order parameter two-point function in the presence of a symmetry breaking wall beyond the leading order in $\varepsilon$. Both the leading short distance behavior proportional to $s^{-2 x_{\varphi}}$ and the nonvanishing exponent of the next to leading power law behavior $s^{-2 x_{\varphi}+x_{\epsilon}}$ of the expansion (2.39) will be identified.

In the half space (hs) system with an ordering field $(+)$ of infinite strength in the planar surface, we introduce the deviation $\phi=\Phi-m$ of the order parameter field $\Phi$ from its mean value $m=\langle\Phi\rangle_{\mathrm{hs}}^{(+)}$. The renormalized and dimensionless two-point function

$$
\mu^{\varepsilon-2}\left\langle\Phi_{\mathrm{R}}\left(\hat{\mathbf{r}}_{1}\right) \Phi_{\mathrm{R}}\left(\hat{\mathbf{r}}_{2}\right)\right\rangle_{\mathrm{hs}}^{(+)}=\tau^{\prime}+\tau^{\prime \prime}
$$

$$
\begin{aligned}
\tau^{\prime} & =\mu^{\varepsilon-2} Z_{\Phi}^{-1} m\left(z_{1}\right) m\left(z_{2}\right) \\
& =\mu^{\varepsilon-2}\left[m_{0}\left(z_{1}\right) m_{0}\left(z_{2}\right)+m_{0}\left(z_{1}\right) m_{I}\left(z_{2}\right)+m_{I}\left(z_{1}\right) m_{0}\left(z_{2}\right)\right]+O\left(u_{\mathrm{R}}\right) \\
& =\frac{3}{4 \pi^{2} u_{\mathrm{R}}} \frac{1}{\left(\mu z_{1}\right)\left(\mu z_{2}\right)} f^{-1}\left\{1+3 \frac{u_{\mathrm{R}}}{\varepsilon}\left[-1+\frac{1}{2}\left[\left(\mu z_{1}\right)^{\varepsilon}+\left(\mu z_{2}\right)^{\varepsilon}\right] \Gamma(1-(\varepsilon / 2))(4 \pi)^{\varepsilon / 2} f \frac{1-\frac{\varepsilon}{6}}{\left.\left.(1+\varepsilon)\left(1+\frac{\varepsilon}{2}\right)\right]\right\}+O\left(u_{\mathrm{R}}\right)}\right.\right.
\end{aligned}
$$

and

$$
\begin{aligned}
\tau^{\prime \prime} & =\mu^{\varepsilon-2} Z_{\Phi}^{-1}\left\langle\phi\left(\hat{\mathbf{r}}_{1}\right) \phi\left(\hat{\mathbf{r}}_{2}\right)\right\rangle_{\mathrm{hs}}^{(+)} \\
& =\mu^{\varepsilon-2} G\left(\hat{\mathbf{r}}_{1 \|}-\hat{\mathbf{r}}_{2 \|} ; z_{1}, z_{2}\right)+O\left(u_{\mathrm{R}}\right),
\end{aligned}
$$

where

$$
\begin{aligned}
G\left(0 ; z_{1}, z_{2}\right)= & \frac{1}{4} \pi^{-2+\varepsilon / 2} \Gamma(1-(\varepsilon / 2))\left\{s^{\varepsilon-2}-(2 z)^{\varepsilon-2}\right. \\
& +\frac{1}{1-\varepsilon}\left[\frac{3}{z_{1} z_{2}}\left(\frac{3}{\varepsilon}-1\right)\left[s^{\varepsilon}+(2 z)^{\varepsilon}\right]\right. \\
& -\frac{3}{\varepsilon}\left(\frac{1}{z_{1}^{2}}+\frac{1}{z_{2}^{2}}\right)\left[s^{\varepsilon}-(2 z)^{\varepsilon}\right] \\
& \left.\left.+\frac{9}{z_{1}^{2} z_{2}^{2}} \frac{1}{\varepsilon(2+\varepsilon)}\left[s^{\varepsilon+2}-(2 z)^{\varepsilon+2}\right]\right]\right\}
\end{aligned}
$$

is the Gaussian propagator of order parameter fluctuations, see Eq. (2.4) in Ref. 39. Here the $z_{i}$ are the distances of $\hat{\mathbf{r}}_{i}$ from the boundary wall, $m_{0}, m_{I}$ are the contributions of order $u^{-1 / 2}$ and $u^{1 / 2}$ to $m$, see Eqs. (2.3) and (4.4) in Ref. 39, and we confine our attention to the case $\hat{\mathbf{r}}_{1 \|}=\hat{\mathbf{r}}_{2 \|}$, where $G$ has the simple form (B28), with $s=\left|z_{1}-z_{2}\right|$ and $2 z=z_{1}$ $+z_{2}$. The factor $Z_{\Phi}=1+O\left(u_{\mathrm{R}}^{2}\right)$ can be neglected to the order considered.
The leading short distance behavior $B_{\varphi} s^{-2 x_{\varphi}}$ on the left hand side of Eq. (2.39) is consistent with the $s^{\varepsilon-2}$ contribution in Eq. (B28). In order to identify the next to leading behavior in Eqs. (B25)-(B28), rewrite $z_{1}, z_{2}$ in terms of $z$ and $s$, consider $\varepsilon \ll 1$, and collect the terms

$$
\begin{aligned}
{\left[\tau^{\prime}, \tau^{\prime \prime}\right]_{O\left(s^{\prime} 0^{\prime}\right)}=} & \frac{3}{4 \pi^{2}} \frac{1}{(\mu z)^{2}}\left[u_{\mathrm{R}}^{-1}\left(1-\varepsilon f_{1}\right)+3 \ln (\mu z)\right. \\
& \left.+\kappa^{\prime}+3 f_{1}, \ln (\mu s)-\ln (\mu z)+\kappa^{\prime \prime}\right]
\end{aligned}
$$

of order $(\mu s)^{0}$ and $\ln (\mu s)$. Here terms of order $u_{\mathrm{R}}^{-1} \varepsilon^{2}, \varepsilon$, and $u_{\mathrm{R}}$ have been neglected, and

$$
\kappa^{\prime}=\frac{3}{2} \ln (4 \pi)-\frac{3}{2} \psi(1)-5, \kappa^{\prime \prime}=\frac{11}{12}-\ln 2
$$

with $\psi$ the logarithmic derivative of the $\Gamma$ function. The corresponding contribution to Eq. (B25),

$$
\begin{aligned}
{\left[\tau^{\prime}+\tau^{\prime \prime}\right]_{O\left(s^{\prime} 0^{\prime}\right)}^{*}=} & \frac{9}{4 \pi^{2} \varepsilon} \frac{1}{(\mu z)^{2}}\left[1+\frac{2}{3} \varepsilon \ln (\mu z)+\frac{1}{3} \varepsilon \ln (\mu s)\right. \\
& \left.+\frac{1}{3} \varepsilon\left(-\frac{17}{9}+\kappa^{\prime}+\kappa^{\prime \prime}\right)+O\left(\varepsilon^{2}\right)\right], \quad(\mathrm{B} 31)
\end{aligned}
$$

at the fixed point $u_{\mathrm{R}}=u_{\mathrm{R}}^{*}=(\varepsilon / 3)[1+(17 / 27) \varepsilon]$, is consistent with the asymptotic critical next to leading behavior 
$\propto(\mu z)^{-x} \epsilon(\mu s)^{x_{\epsilon}-2 x_{\varphi}}$ of $\langle\varphi \varphi\rangle_{\mathrm{hs}}$, as suggested by Eq. (2.39), since for $\varepsilon$ small, $2-x_{\epsilon} \rightarrow(2 / 3) \varepsilon$, and $x_{\epsilon}-2 x_{\varphi}=-\eta_{t}^{*}$ $-\eta_{\Phi}^{*} \rightarrow \varepsilon / 3$, see Eq. (B18).

\section{APPENDIX C: MULTIPOINT AVERAGES IN THE GAUSSIAN MODEL}

First we check that the explicit expressions for the short distance behavior of the order parameter and energy density two-point averages near an arbitrary interior point of a Gaussian model between parallel plates with Dirichlet boundary conditions are consistent with the operator product expansion (2.39). For the order parameter average at the bulk critical temperature,

$$
\begin{aligned}
& \left\langle\Phi\left(\hat{\mathbf{r}}_{1}\right) \Phi\left(\hat{\mathbf{r}}_{2}\right)\right\rangle_{\mathrm{film}}-\left\langle\Phi\left(\hat{\mathbf{r}}_{1}\right) \Phi\left(\hat{\mathbf{r}}_{2}\right)\right\rangle_{\text {bulk }} \\
& =\int \frac{d^{d-1} p}{(2 \pi)^{d-1}}\left\{\frac{\sinh \left[p\left(L+\hat{z}_{\min }\right)\right] \sinh \left[p\left(L-\hat{z}_{\max }\right)\right]}{p \sinh (2 p L)}\right. \\
& \left.\quad-\frac{\exp \left[-p\left|\hat{z}_{1}-\hat{z}_{2}\right|\right]}{2 p}\right\} e^{i \hat{\mathbf{p}}_{12 \|}}
\end{aligned}
$$

with $\hat{z}_{\min }=\min \left(\hat{z}_{1}, \hat{z}_{2}\right), \hat{z}_{\max }=\max \left(\hat{z}_{1}, \hat{z}_{2}\right)$, one finds

$$
\begin{aligned}
\left(\widetilde{S}_{d}\right)^{-1}\left\langle\Phi\left(\hat{\mathbf{r}}_{1}\right) \Phi\left(\hat{\mathbf{r}}_{2}\right)\right\rangle_{\text {film }}-s^{2-d} \\
\quad \rightarrow(2 L)^{2-d}[\sqrt{2} \mathcal{E}(y)-v+w]
\end{aligned}
$$

with

$$
\begin{aligned}
& v=\sqrt{2} \mathcal{E}^{\prime \prime}(y) \mathbf{s}_{\|}^{2} /\left[8(d-1) L^{2}\right], \\
& w=\left[\mathbf{s}_{\|}^{2}-(d-1) s_{\perp}^{2}\right] \Delta_{\text {ord,ord }} /\left(8 \widetilde{S}_{d} L^{2}\right),
\end{aligned}
$$

$y=\left(\hat{z}_{1}+\hat{z}_{2}\right) /(2 L), s_{\perp}=\hat{z}_{1}-\hat{z}_{2}, \mathbf{s}_{\|}^{2}=\hat{\mathbf{r}}_{12}^{2}-s_{\perp}^{2}$. Here $\mathcal{E}$ is the scaling function (3.37) of the energy density profile, and $\Delta_{\text {ord,ord }}$ is the amplitude in Eq. (3.35) of the stress tensor average. The terms in Eq. (C2) beyond the bulk contribution $s^{2-d}$ are of order $s^{0}$, and $\mathbf{s}_{\|}^{2}, s_{\perp}^{2}$ and can be identified with corresponding terms in the film average of the product expansion (2.39) for $\mathcal{O}=\varphi$ on using the three-point universal amplitudes

$$
\frac{C_{\varphi \varphi \epsilon}}{B_{\varphi} B_{\epsilon}^{1 / 2}}=\sqrt{2}, \quad \frac{C_{\varphi \varphi T}}{B_{\varphi} B_{T}}=-\frac{1}{2 \widetilde{S}_{d}}, C_{\varphi \varphi \lambda}=0 .
$$

For the energy two-point average in the Gaussian model, Wick's theorem

$$
\begin{aligned}
\left\langle\Phi^{2}\left(\hat{\mathbf{r}}_{1}\right) \Phi^{2}\left(\hat{\mathbf{r}}_{2}\right)\right\rangle_{\text {film }}= & \left\langle\Phi^{2}\left(\hat{\mathbf{r}}_{1}\right)\right\rangle_{\text {film }}\left\langle\Phi^{2}\left(\hat{\mathbf{r}}_{2}\right)\right\rangle_{\text {film }} \\
& +2\left[\left\langle\Phi\left(\hat{\mathbf{r}}_{1}\right) \Phi\left(\hat{\mathbf{r}}_{2}\right)\right\rangle_{\text {film }}\right]^{2}
\end{aligned}
$$

and Eq. (C2) imply

$$
\begin{aligned}
\left(2 \widetilde{S}_{d}^{2}\right)^{-1}\left\langle\Phi^{2}\left(\hat{\mathbf{r}}_{1}\right) \Phi^{2}\left(\hat{\mathbf{r}}_{2}\right)\right\rangle_{\mathrm{film}}-s^{2(2-d)} & \\
\quad \rightarrow & 2^{3 / 2}(2 L)^{2-d} \mathcal{E}(y) s^{2-d}+3(2 L)^{4-2 d}[\mathcal{E}(y)]^{2} \\
& +2(2 L)^{2-d}[-v+w] s^{2-d} .
\end{aligned}
$$

The short distance contributions beyond the bulk contribution $s^{2(2-d)}$ are of order $s^{2-d}, s^{0}$, and $\left(\mathbf{s}_{\|}^{2}, s_{\perp}^{2}\right) s^{2-d}$ and can be identified with those in the film average of Eq. (2.39) with
$\mathcal{O}=\epsilon$. Here one uses the relation between $\lambda$ and $\Phi^{4}$ given just before Eq. (3.39) and the three-point universal amplitude values

$$
\frac{C_{\epsilon \epsilon \epsilon}}{B_{\epsilon}^{3 / 2}}=2^{3 / 2}, \frac{C_{\epsilon \epsilon T}}{B_{\epsilon} B_{T}}=-\frac{1}{\widetilde{S}_{d}}, \frac{C_{\epsilon \epsilon \lambda}}{B_{\epsilon} B_{\lambda}^{1 / 2}}=\sqrt{6} .
$$

Finally we consider the four-point average

$$
\begin{aligned}
& \left\langle\Phi\left(\mathbf{r}_{1}\right) \Phi\left(\mathbf{r}_{2}\right) \Phi\left(\mathbf{r}_{3}\right) \Phi\left(\mathbf{r}_{4}\right)\right\rangle_{\mathrm{DB}} \\
& \quad=\psi_{1,2} \psi_{3,4}+\psi_{1,3} \psi_{2,4}+\psi_{1,4} \psi_{2,3}
\end{aligned}
$$

with $\psi_{1,2}=\left\langle\Phi\left(\mathbf{r}_{1}\right) \Phi\left(\mathbf{r}_{2}\right)\right\rangle_{\text {DB }}$ in the presence of the dumbbell. The contribution of order $L^{2(d-2)}$ to the left hand side of Eq. (C8) comes from the operator $a_{3} \lambda$ in Eq. (3.38) and is given by $6(L / 2)^{2(d-2)} \mathcal{E}^{2}(0) \widetilde{S}_{d}^{2} /\left(r_{1} r_{2} r_{3} r_{4}\right)^{d-2}$. This is consistent with the corresponding contribution $\left\langle\Phi\left(\mathbf{r}_{1}\right) \Phi\left(\mathbf{r}_{2}\right) a_{1} \epsilon\right\rangle_{\text {bulk }}\left\langle\Phi\left(\mathbf{r}_{3}\right) \Phi\left(\mathbf{r}_{4}\right) a_{1} \epsilon\right\rangle_{\text {bulk }}+\cdot+$. to the right-hand side of Eq. (C8), where $a_{1} \epsilon$ is from Eq. (3.31). Note that $\psi$ does not contain a contribution of order $L^{2(d-2)}$, since $C_{\varphi \varphi \lambda}$ vanishes in the Gaussian model.

\section{APPENDIX D: TWO-POINT FUNCTIONS IN THE $d=2$ ISING MODEL}

For the symmetric dumbbells of Figs. 1(a) and 1(b), one finds the two-point averages

$$
\begin{aligned}
& \left\langle\varphi\left(\mathbf{r}_{1}\right) \varphi\left(\mathbf{r}_{2}\right)\right\rangle_{\mathrm{DB}}^{(i, i)} / B_{\varphi} \\
& \quad=\left(\mathcal{A}_{\varphi}^{(+)}\right)^{2}\left(\frac{\Lambda_{1} \Lambda_{2}}{c_{1} c_{2}}\right)^{1 / 8} 2^{-1 / 2}\left(u \pm u^{-1}\right)^{1 / 2}
\end{aligned}
$$

with upper and lower signs for $(i, i)=(+,+)$ and (ord,ord), and

$$
\begin{aligned}
\left\langle\epsilon\left(\mathbf{r}_{1}\right) \epsilon\left(\mathbf{r}_{2}\right)\right\rangle_{\mathrm{DB}}^{(i, i)} / B_{\epsilon}= & \left(\mathcal{A}_{\epsilon}^{(+)}\right)^{2} \Lambda_{1} \Lambda_{2} 4 \\
& \times\left(\frac{1}{4 c_{1} c_{2}}+\frac{1}{C}-\frac{1}{C+4 c_{1} c_{2}}\right)
\end{aligned}
$$

for both $(i, i)=(+,+)$ and (ord,ord), and

$$
\begin{aligned}
& \left\langle\varphi\left(\mathbf{r}_{1}\right) \epsilon\left(\mathbf{r}_{2}\right)\right\rangle_{\mathrm{DB}}^{(+,+)} /\left(B_{\varphi} B_{\epsilon}\right)^{1 / 2} \\
& \quad=\mathcal{A}_{\varphi}^{(+)} \mathcal{A}_{\epsilon}^{(+)}\left(\frac{\Lambda_{1}}{c_{1}}\right)^{1 / 8} \frac{\Lambda_{2}}{c_{2}}\left(1+\frac{4 c_{1} c_{2}}{C}\right)^{-1 / 2}\left(1+\frac{2 c_{1} c_{2}}{C}\right) .
\end{aligned}
$$

For the $(-,+)$ dumbbell of Fig. 1(c),

$$
\begin{aligned}
& \left\langle\varphi\left(\mathbf{r}_{1}\right) \varphi\left(\mathbf{r}_{2}\right)\right\rangle_{\mathrm{DB}}^{(-,+)} / B_{\varphi} \\
& =\left(\mathcal{A}_{\varphi}^{(+)}\right)^{2}\left(\frac{\Lambda_{1} \Lambda_{2}}{c_{1} c_{2}}\right)^{1 / 8} 2^{-1 / 2}\left[\left(u+u^{-1}\right)^{1 / 2} s_{1} s_{2}\right. \\
& \left.\quad+\frac{u-u^{-1}}{\left(u+u^{-1}\right)^{1 / 2}} c_{1} c_{2}\right]
\end{aligned}
$$




$$
\begin{aligned}
\left\langle\epsilon\left(\mathbf{r}_{1}\right)\right. & \left.\epsilon\left(\mathbf{r}_{2}\right)\right\rangle_{\mathrm{DB}}^{(-,+)} / B_{\epsilon} \\
= & \left(\mathcal{A}_{\epsilon}^{(+)}\right)^{2} \Lambda_{1} \Lambda_{2} 4\left\{\frac{1}{4 c_{1} c_{2}}\left(1-4 c_{1}^{2}\right)\left(1-4 c_{2}^{2}\right)\right. \\
& +\frac{1}{C}\left[1-4 \sin ^{2}\left(\theta_{1}-\theta_{2}\right)\right]-\frac{1}{C+4 c_{1} c_{2}} \\
& \left.\times\left[1-4 \sin ^{2}\left(\theta_{1}+\theta_{2}\right)\right]\right\},
\end{aligned}
$$

and

$$
\begin{aligned}
\left\langle\varphi\left(\mathbf{r}_{1}\right)\right. & \left.\epsilon\left(\mathbf{r}_{2}\right)\right\rangle_{\mathrm{DB}}^{(-,+)} /\left(B_{\varphi} B_{\epsilon}\right)^{1 / 2} \\
= & \mathcal{A}_{\varphi}^{(+)} \mathcal{A}_{\epsilon}^{(+)}\left(\frac{\Lambda_{1}}{c_{1}}\right)^{1 / 8} \frac{\Lambda_{2}}{c_{2}}\left(1+\frac{4 c_{1} c_{2}}{C}\right)^{-1 / 2} \\
& \times\left[\left(1+\frac{2 c_{1} c_{2}}{C}\right) s_{1}\left(1-4 c_{2}^{2}\right)+4 \frac{c_{1}^{2} c_{2}}{C} \sin \left(2 \theta_{2}\right)\right] .
\end{aligned}
$$

Here

$$
\begin{aligned}
& \Lambda_{k}=\frac{\pi L}{2 r_{k}^{2}}, \quad \theta_{k}=\Lambda_{k} r_{k \|}, \quad c_{k}=\cos \theta_{k}, \quad s_{k}=\sin \theta_{k}, \\
& C=2\left[\cosh \left(\Lambda_{1} r_{1 \perp}-\Lambda_{2} r_{2 \perp}\right)-\cos \left(\theta_{1}-\theta_{2}\right)\right], \\
& u=\left(1+\frac{4 c_{1} c_{2}}{C}\right)^{1 / 4} .
\end{aligned}
$$

One may check the surface limit of these expressions most easily for $\mathbf{r}_{1}$ and $\mathbf{r}_{2}$ approaching, respectively, the lowest and highest surface point of the dumbbell in Fig. 1, for which

$r_{1 \perp}=r_{2 \perp}=0, r_{1 \|}=-L-\delta_{1} \nearrow-L, r_{2 \|}=L+\delta_{2} \searrow L$,

$c_{k} \rightarrow \pi \delta_{k} /(2 L), \quad C \rightarrow 4, \quad \Lambda_{k} / c_{k} \rightarrow 1 / \delta_{k}, \quad s_{1} \rightarrow-1, s_{2} \rightarrow 1$.

For the symmetric dumbbells with $(i, i)=(+,+)$ or (ord, ord), one obtains

$$
\left\langle\mathcal{O}_{1} \mathcal{O}_{2}\right\rangle_{\mathrm{DB}}^{(i, i)} /\left(B_{\mathcal{O}_{1}} B_{\mathcal{O}_{2}}\right)^{1 / 2} \rightarrow \mathcal{A}_{\mathcal{O}_{1}}^{(i)} \mathcal{A}_{\mathcal{O}_{2}}^{(i)} /\left(\delta_{1}^{x_{\mathcal{O}_{1}}} \delta_{2}^{\left.x_{\mathcal{O}_{2}}\right)},\right.
$$

where $\mathcal{O}_{k}=\varphi$ or $\epsilon$, and for the $(-,+)$ dumbbell

$$
\begin{aligned}
& \left\langle\varphi \mathcal{O}_{2}\right\rangle_{\mathrm{DB}}^{(-,+)} /\left(B_{\varphi} B_{\mathcal{O}_{2}}\right)^{1 / 2} \rightarrow-\mathcal{A}_{\varphi}^{(+)} \mathcal{A}_{\mathcal{O}_{2}}^{(+)} /\left(\delta_{1}^{x_{\varphi}} \delta_{2}^{\left.x_{\mathcal{O}_{2}}\right),}\right. \\
& \langle\epsilon \epsilon\rangle_{\mathrm{DB}}^{(-,+)} / B_{\epsilon} \rightarrow\left(\mathcal{A}_{\epsilon}^{(+)}\right)^{2} /\left(\delta_{1} \delta_{2}\right) .
\end{aligned}
$$

From the order parameter three-point function in the half space with a $(+)$ boundary, ${ }^{25}$ one finds, for a $(+,+)$ dumbbell,

$$
\begin{aligned}
\left\langle\varphi\left(\mathbf{r}_{1}\right)\right. & \left.\varphi\left(\mathbf{r}_{2}\right) \varphi\left(\mathbf{r}_{3}\right)\right\rangle_{\mathrm{DB}}^{(+,+)} / B_{\varphi}^{3 / 2} \\
= & \left(\mathcal{A}_{\varphi}^{(+)}\right)^{3}\left(\frac{\Lambda_{1} \Lambda_{2} \Lambda_{3}}{c_{1} c_{2} c_{3}}\right)^{1 / 8} 2^{-3 / 2} \\
& \times\left[\left(u_{12}+u_{12}^{-1}\right)\left(u_{23}+u_{23}^{-1}\right)\left(u_{31}+u_{31}^{-1}\right)\right. \\
& \left.-\left(u_{12}-u_{12}^{-1}\right)\left(u_{23}-u_{23}^{-1}\right)\left(u_{31}-u_{31}^{-1}\right)\right]^{1 / 2} .
\end{aligned}
$$

Here $u_{12}$ is given by $u$ in Eq. (D7), and $u_{23}$ and $u_{31}$ follow on replacing $c_{1} c_{2}$ in Eq. (D7) by $c_{2} c_{3}$ and $c_{3} c_{1}$ and the subscripts 1,2 in $C$ by 2,3 and 3,1 .
${ }^{1}$ M. E. Fisher and P. G. de Gennes, C. R. Acad. Sci. Paris, 287, B-207 (1978).

${ }^{2}$ P. G. de Gennes, C. R. Acad. Sci., Paris, 292, II-701 (1981).

${ }^{3}$ K. G. Wilson and J. Kogut, Phys. Rep. 12, 75 (1974).

${ }^{4} \mathrm{~K}$. Binder, in Phase Transitions and Critical Phenomena, edited by C. Domb and J. L. Lebowitz (Academic, London, 1983), Vol. 8.

${ }^{5}$ (a) H. W. Diehl, in Phase Transitions and Critical Phenomena, edited by C. Domb and J. L. Lebowitz (Academic, London, 1986), Vol. 10; (b) Int. J. Mod. Phys. B 11, 3503 (1997).

${ }^{6}$ T. Burkhardt and E. Eisenriegler, J. Phys. A 18, L83 (1985).

${ }^{7}$ T. Burkhardt and E. Eisenriegler, Phys. Rev. Lett. 74, 3189 (1995); 78, 2867 (1997).

${ }^{8}$ E. Eisenriegler and U. Ritschel, Phys. Rev. B 51, 13717 (1995).

${ }^{9}$ A. Hanke, F. Schlesener, E. Eisenriegler, and S. Dietrich, Phys. Rev. Lett. 81, 1885 (1998).

${ }^{10}$ F. Schlesener, A. Hanke, and S. Dietrich, J. Stat. Phys. 110, 981 (2003).

${ }^{11}$ B. M. Law, J. M. Petit, and D. Beysens, Phys. Rev. E 57, 5782 (1998).

${ }^{12}$ P. G. de Gennes, Scaling Concepts in Polymer Physics (Cornell University, Ithaca, 1979).

${ }^{13}$ (a) E. Eisenriegler, Polymers Near Surfaces (World Scientific, Singapore, 1993); (b) in Field Theoretical Tools in Polymer-and Particle-Physics, edited by H. Meyer-Ortmanns and A. Klümper, Lecture Notes in Physics Vol. 508 (Springer, Berlin, 1998).

${ }^{14}$ M. A. Bates and D. Frenkel, Phys. Rev. E 62, 5225 (2000).

${ }^{15}$ F. M. van der Kooij, M. Vogel, and H. N. W. Lekkerkerker, Phys. Rev. E 62, 5397 (2000)

${ }^{16}$ E. Eisenriegler, A. Bringer, and R. Maassen, J. Chem. Phys. 118, 8093 (2003).

${ }^{17}$ A. Hanke, E. Eisenriegler, and S. Dietrich, Phys. Rev. B 59, 6853 (1999).

${ }^{18}$ In particular, we consider a particle size much larger than the extrapolation length $\ell$ of the particle surface (Refs. 1, 2, 4, and 5). We do not discuss crossover phenomena which may occur for particle surfaces with a weak preference for one of the two solvent phases and where $\ell$ is of mesoscopic size. To estimate the effect of the extrapolation length on density profiles or correlation functions, consider them for fixed point conditions but for a boundary surface which is shifted by $\ell$. For a symmetric dumbbell of two touching spheres with positive $\ell$, thus take the surfaces of two slightly overlapping spheres with a diameter $L+2 \ell$ and center-to-center distance $L$, intersecting in a circle with diameter $\mathcal{D}=2(\ell L)^{1 / 2}$, and at an angle $\alpha$ $=4(\ell / L)^{1 / 2}$, see Eqs. (5.11) and (5.12). Concentrating, for simplicity, on the symmetry plane $r_{\|}=0$ of the dumbbell, the normalized profile $\langle\mathcal{O}(\mathbf{r})\rangle / B_{\mathcal{O}}$ is then given by the product of $\mathcal{D}^{x} \mathcal{O} \overline{\mathcal{P}}(\alpha, 0)$ and $\left[r_{\perp}^{2}\right.$ $\left.-(\mathcal{D} / 2)^{2}\right]^{-x_{\mathcal{O}}}$, see Eq. (5.14). This confirms the expectation that $\ell$ drops out and the fixed point profile (2.28) of two touching spheres is reproduced provided (i) the particle size $L$ is much larger than $\ell$ so that the first factor tends to $(L / 2)^{x} \mathcal{O} \mathcal{P}(0)$, see Eq. (5.11), (5.3), or (5.8) and (ii) $\mathbf{r}$ is much farther from the sphere surfaces than $\ell$ so that $r_{\perp} \gg \mathcal{D}$ and the second factor tends to $r_{\perp}^{-2 x_{\mathcal{O}}}$.

${ }^{19}$ Anisotropic operators have been considered before in Ref. 16 to describe ideal polymers interacting with small anisotropic particles.

${ }^{20}$ E. Snoeks et al., Adv. Mater. (Weinheim, Ger.) 12, 1511 (2000).

${ }^{21}$ A. van Blaaderen, Science 301, 470 (2003); MRS Bulletin, February, 85 (2004).

${ }^{22}$ C. M. van Kats, P. M. Johnson, and A. van Blaaderen, Colloidal Dumbbells in Real Space, Poster at the Juelich Soft Matter Days, November 2003.

${ }^{23}$ J. L. Cardy, in Phase Transitions and Critical Phenomena, edited by C. Domb and J. L. Lebowitz (Academic, London, 1986), Vol. 11, p. 55.

${ }^{24}$ J. L. Cardy, Nucl. Phys. B 290, 355 (1987).

${ }^{25}$ (a) T. W. Burkhardt and T. Xue, Phys. Rev. Lett. 66, 895 (1991); (b) Nucl. Phys. B 354, 653 (1991); (c) T. W. Burkhardt and I. Guim, Phys. Rev. B 36, 2080 (1987); (d) 47, 14306 (1993).

${ }^{26}$ M. Krech, Phys. Rev. E 56, 1642 (1997).

${ }^{27}$ See, e.g., C. G. Callan, in Methods in Field Theory, edited by R. Balian and J. Zinn-Justin (North-Holland, Amsterdam, 1976), Session 26, pp. $41-77$.

${ }^{28}$ (a)L. S. Brown, Ann. Phys. (N.Y.) 126, 135 (1980); (b) L. S. Brown and J. C. Collins, ibid. 130, 215 (1980).

${ }^{29}$ We always consider the asymptotic long-distance limit (Ref. 18), which 
corresponds to the fixed points $-\infty$ and $+\infty$ of the symmetry breaking surface "magnetic" fields on the two spheres of Fig. 1(c).

${ }^{30}$ This relation between the free energy and the stress tensor average (2.16) of the film specifies our normalization of the stress tensor. It coincides with the normalization in Ref. 28 and implies that the integral $\int d^{d-1} r_{\|}^{\prime}$ of the average in the half space $\left\langle T_{z z}\left(\mathbf{r}_{\|}^{\prime}, z^{\prime}\right) \mathcal{O}\left(\mathbf{r}_{\|}, z\right)\right\rangle_{\text {hs }}$ over a plane with distance $z^{\prime}$ from the surface which is smaller than the distance $z$ equals the derivative $\partial_{z}\left\langle\mathcal{O}\left(\mathbf{r}_{\|}, z\right)\right\rangle_{\mathrm{hs}}$.

${ }^{31}$ D. M. Mc Avity and H. Osborn, Nucl. Phys. B 455, 522 (1995).

${ }^{32}$ For a sphere the small particle expansion has been checked for temperatures above $T_{c}$, see, e.g., Refs. 9, 16, and 17.

${ }^{33}$ Besides $\partial_{\mu} \partial_{\nu} \epsilon$ and $T_{\mu \nu}$ from Refs. 31 and 24 we also explicitly consider the operator $\lambda$ in Eq. (2.39) which near $d=4$ leads to the same order in the small distance.

${ }^{34}$ For our definition (Ref. 30) of the stress tensor $T_{\mu, \nu}$, the complex component $T$ equals $-\pi\left(T_{x x}-i T_{x y}\right)$, and $\bar{T}$ equals $-\pi\left(T_{x x}+i T_{x y}\right)$.

${ }^{35}$ J. Chen and A. Anandarajah, J. Colloid Interface Sci. 180, 519 (1996).

${ }^{36}$ M. Krech (unpublished).

${ }^{37}$ This interpolation leads to $\left(2^{x} \mathcal{A}_{\varphi}^{(+)}\right)^{2} \equiv a=7.34$, close to the value $a$ $=7.73$ obtained in Ref. 9 by a different interpolation.

${ }^{38}$ A. Hanke, M. Krech, F. Schlesener, and S. Dietrich, Phys. Rev. E 60, 5163 (1999).

${ }^{39}$ E. Eisenriegler and M. Stapper, Phys. Rev. B 50, 10009 (1994). 